\title{
A frequency count of 190,000 words in the London-Lund Corpus of English Conversation
}

\author{
GORDON D. A. BROWN \\ University of Essex, Colchester, England
}

\begin{abstract}
A frequency count of more than 190,000 words of spoken English is presented. The count is based on a published corpus of spontaneous conversation (Svartvik \& Quirk, 1980). A brief description of the count is presented, and the correlations between spoken word frequency and a range of other word variables are reported. It is expected that the frequency count will be useful in the interpretation of certain psychological data.
\end{abstract}

It is well known that the frequency of occurrence of a written word is a good predictor of the recognition time for that word (e.g., Whaley 1978). However, the influence of spoken word frequency on word naming and other tasks has been less thoroughly investigated. This is due in part to the lack of a suitable frequency count of words in the spoken language. Rubin (1980) and Whaley (1978) have reported major multiple regression analyses of factors influencing verbal behavior, but neither study included a measure of spoken word frequency. If such a measure had been included, it might have been possible to show that the apparently independent influence of certain factors, such as age of acquisition for words, was redundant on spoken word frequency. Tryk (1968) claimed that spoken and written frequency represent distinct, although correlated, variables, and Gernsbacher (1984) claimed that rated familiarity provides a better measure of "experiential familiarity" than objective written word frequency measures do. It is possible that the spoken word frequency count presented here may provide a more adequate measure for use in psychological experimentation.

Increasing use of computers as a storage medium for text means that word frequency measures based on very large samples of written language may soon become available, but it will probably be several years before counts of frequency of occurrence in the spoken language can be produced without large amounts of laborious transcription. The count presented here is based on a set of published transcriptions of spontaneous conversation (Svartvik \& Quirk, 1980).

Previously published counts based on the spoken langauge have relied on sources as varied as telephone conversations (French, Carter, \& Koenig, 1930) and proverb interpretations (Fairbanks, 1944). A larger and more recent count (Howes, 1966) is based on 250,000 words of recorded interviews with university students and hospital patients. However, the study suffers from the disadvantage that the interviewees knew that their speech was being recorded to provide a statistical sample of language; indeed, this was the primary purpose of the interviews. In contrast, in the corpus

The work reported here was carried out while the author was in receipt of a SERC grant at the University of Sussex (Laboratory of Experimental Psychology). I am grateful to Yumi Hanstock and Alan Richomme for technical assistance, and to Professor Quirk for permission to publish the frequency count. University College, London, and the University of Lund made the corpus available through the Norwegian Computing Centre for the Humanities. Mailing address: Department of Language and Linguistics, University of Essex, Colchester CO4 3SQ, England. analyzed in the present paper, the vast majority of words were spoken by people who did not know that their responses were being recorded.

\section{SOURCE OF DATA}

The data were obtained from the book $A$ Corpus of English Conversation (Svartvik \& Quirk, 1980). This book contains transcriptions of 34 "texts," each of which contains 5,000 words spoken by people unaware that recording was taking place, together with a relatively small number of words uttered by speakers aware of the recording. All of the speakers were educated native speakers of English. Further details about the conditions under which the recordings were made can be obtained from Svartvik and Quirk. The total number of words in the published version of the corpus is 191,918 , and the corpus contains approximately 10,630 different words (including proper names).

\section{METHOD OF ANALYSIS}

The analysis was performed from a machine-readable tape containing all the published transcripts. All information about stress, pause duration, speaker, etc., was removed, leaving only the words themselves. Many names of people and places had been changed in the published versions of the transcripts in order to preserve anonymity, and it was therefore necessary to remove these items. Because it was impossible to determine which names had been replaced, it was decided to adopt a conservative criterion and remove all names of people and places. This accounted for the removal of 1,615 different items, leaving 9,018 words in the count. These different items accounted for 15,658 tokens in the transcript, but of course it remains the case that the figures in the accompanying appendices represent frequency of occurrence per 191,918. Finally, entries that differed only in letter case were combined, and a frequency count was produced using a PDP-11/40 computer. This is listed as Appendix A. In order to preserve page space, Appendix A does not include 4,073 words that occurred only once in the corpus (a listing of these words is available from the author on request). Appendix $B$ is a listing of all the words in the corpus that occurred with a frequency greater than 150 .

\section{SUMMARY STATISTICS}

A correlational analysis of a sample of the word frequency count was performed, in order to assess the rela- 
tion between spoken word frequency and other word variables. Because it was impracticable to obtain a range of word attribute measures for such a large sample of words, use was made of previously published ratings. Gilhooly and Logie (1980) reported ratings of a sample of 1,944 words on a number of different dimensions, including rated familiarity, age of acquisition, imageability, concreteness, and ambiguity. Every word that appeared in both the Gilhooly and Logie ratings and the present frequency count was selected. This resulted in a sample of 437 items. The sample was further reduced, however, in order to enable a measure of orthographic regularity to be included in the correlational analysis. The best available measure of this type is the positional bigram frequency count published by Solso and Juel (1980), but this count gives values only for words up to 9 letters in length. The 21 words with 10 or more letters were therefore dis carded from the sample, leaving a final sample of 416 items. Summary statistics for this sample appear in Table 1.

Three of the measures displayed skews greater than 1.0, and these skews were successfully reduced by transformation. The two word frequency measures were submitted to $\log 10$ transformations, and the skew in the positional bigram frequency measure was reduced to below 1.0 by a square-root transformation. Following these transformations, an intercorrelation matrix was produced; this appears in Table 2. Most of the correlations were unsurprising. Spoken and written frequency were correlated 0.7 ( $p<.01)$ and displayed broadly similar patterns of correlation with other variables. Both word frequency measures correlate positively and significantly with rated familiarity, although spoken word frequency correlates more highly. Both correlate positively with ambiguity ( $p<.05$ in both cases) and bigram frequency $(p<.01$ in both cases) and negatively with rated concreteness ( $p<.01$ for written frequency; $p<.05$ for spoken frequency). The frequency measures display unexpectedly small (and nonsignificant) correlations with word length; Kučera and Francis (1967) frequency and word length correlated 0.30 in the Rubin (1980) study. Written word frequency correlates positively but nonsignificantly with rated word learning age, whereas spoken word frequency displays a significant $(p<.01)$ negative correlation with this measure. Rated imageability is correlated negatively with both written and spoken word frequency $(\mathrm{p}<.01$

Table 1

Summary Statistics of Variables Included in Correlational Analysis

\begin{tabular}{lrrrl}
\hline \multicolumn{1}{c}{ Variable } & Mean & Range & \multicolumn{1}{c}{ SD } & Transformation \\
\hline Imageability & 4.91 & 4.32 & 1.02 & \\
Age of Acquisition & 3.36 & 4.31 & 0.92 & \\
Familiarity & 5.65 & 3.22 & 0.55 & \\
Concreteness & 4.63 & 4.83 & 1.33 & \\
Ambiguity & 0.47 & 2.36 & 0.59 & \\
Length (Letters) & 5.62 & 6.00 & 1.67 & \\
Bigram Frequency & 1345 & 9461 & 1029 & Square root \\
Spoken Frequency & 18.9 & 532 & 40.2 & Log 10 \\
Written Frequency & 132 & 974 & 132 & Log 10 \\
\hline
\end{tabular}

Table 2

Intercorrelation Matrix With Spoken Word Frequency and Other Word Variables

\begin{tabular}{lrrrrrrrr} 
& 2 & \multicolumn{1}{c}{3} & 4 & 5 & 6 & 7 & \multicolumn{1}{c}{8} & 9 \\
\hline 1) Spoken Frequency & 70 & -12 & 50 & -10 & 11 & 06 & 22 & -13 \\
2) Written Frequency & & 04 & 37 & -16 & 13 & 03 & 20 & -18 \\
3) Age of Acquisition & & & -58 & -58 & 09 & 51 & -13 & -65 \\
4) Familiarity & & & & 21 & -06 & -21 & 17 & 24 \\
5) Concreteness & & & & & -02 & 30 & 05 & 85 \\
6) Ambiguity & & & & & & 14 & 03 & 17 \\
7) Word Length & & & & & & 13 & 29 \\
8) Bigram Frequency & & & & & & & 02 \\
9) Imageability & & & & & & &
\end{tabular}

in both cases). A correlation matrix of this type can of course only be suggestive, owing to the post hoc nature of the observations. Table 2 displays a rank ordering of all (117) words in the present frequency count with frequencies greater than 150. A comparison with the rank ordering in Kučra and Francis (1967) reveals that, of the 100 most frequent words in the present count, 68 are among the 100 most frequent words in Kucera and Francis. Only 6 of the 100 most frequent words listed in the present paper have frequencies of less than 500 per million in the Kucera and Francis count. These words are "YES," “OH," "YEAH," "THAT'S," "SORT," "I'VE," and "HE'S."

There is, of course, considerable scope for further analysis of the ways in which spoken and written word frequency differ. The count presented here should prove useful in the design and interpretation of certain psychology experiments.

\section{REFERENCES}

FaIRBANKS, A. (1944). The quantitative differentiation of samples of spoken language. Psychology Monographs, 56, 19-36.

French, N., CARTer, C. W., \& Koenig, W. (1930). The words and sounds of telephone conversations. Bell Systems Technical Journal, 9, 290-324

GerNSBACHER, M. A. (1984). Resolving 20 years of inconsistent interactions between lexical familiarity and orthography, concreteness and polysemy. Journal of Experimental Psychology: General, 113, 256-281.

GilhoOLY, K. J., \& LogIE, R. H. (1980). Age-of-acquisition, imagery, concreteness, familiarity, and ambiguity measures for 1,944 words. Behavior Research Methods \& Instrumentation, 12, 395-427.

HowEs, D. (1966). A word count of spoken English. Journal of Verbal Learning and Verbal Behavior, 5, 572-604.

KuČERA, H., \& FranCIS, W. H. (1967). Computational analysis of present-day American English. Providence, RI: Brown University Press.

RuBin, D. C. (1980). 51 properties of 125 words: A unit analysis of verbal behavior. Journal of Verbal Learning and Verbal Behavior, 19, 736-755.

Solso, R. L., \& Juel, C. L. (1980). Positional frequency and versatility of bigrams for two- through nine-letter English words. Behavior Research Methods \& Instrumentation, 12, 297-343.

SVARTVIK, J., \&uIRK, R. (1980). A corpus of English conversation. Lund, Sweden: Gleerup.

Tryk, H. E. (1968). Subjective scaling of word frequency. American Journal of Psychology, 81, 170-177.

WhaLEY, C. P. (1978). Word-nonword classification time. Joumal of Verbal Learning and Verbal Behavior, 17, 143-154 
Appendix A

Alphabetical Listing of Words Occurring More Than Once in Svartvik and Quirk (1980)

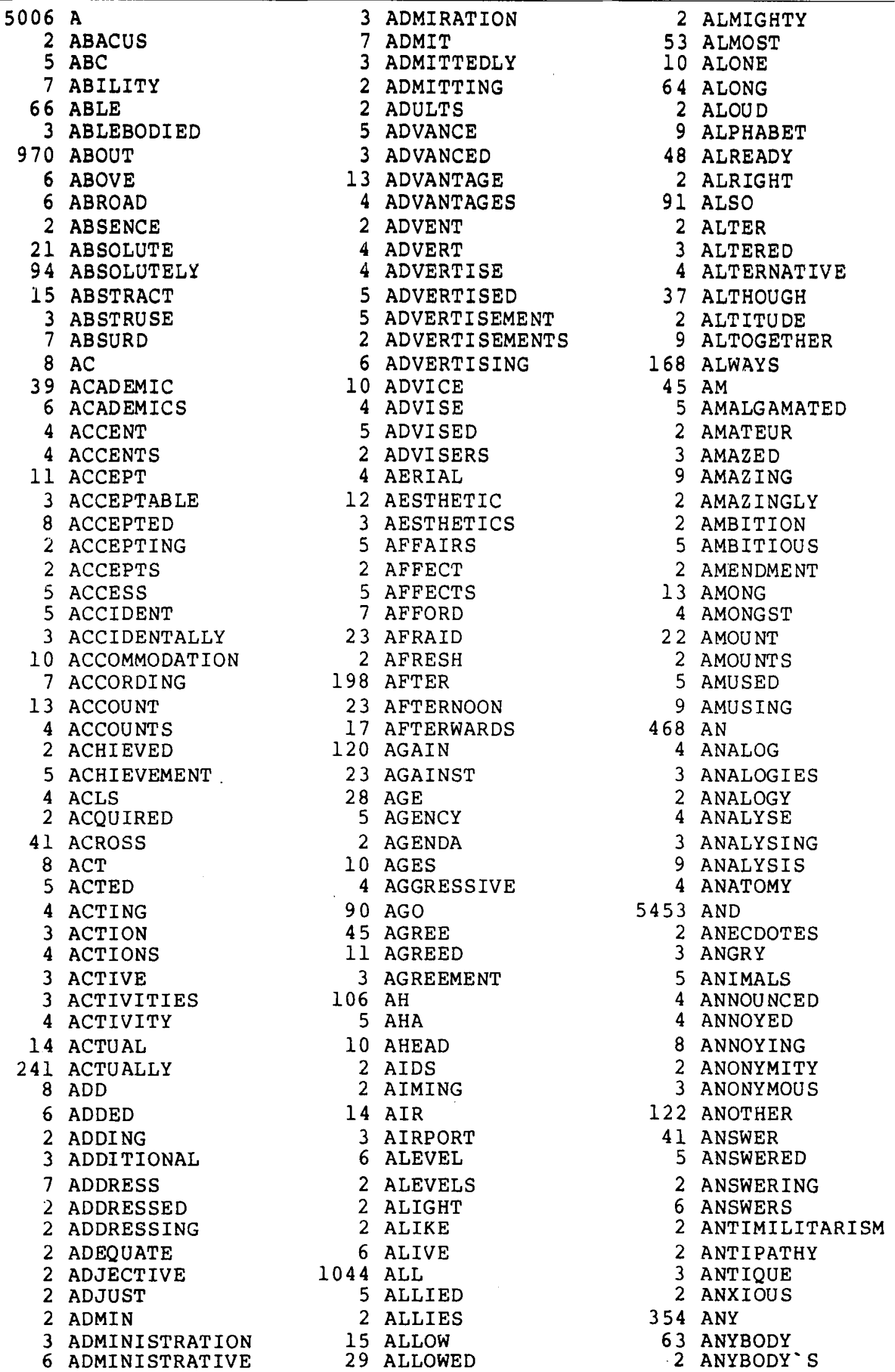


Appendix A (Continued)

\begin{tabular}{|c|c|c|c|c|c|}
\hline $\begin{array}{r}6 \\
16\end{array}$ & $\begin{array}{l}\text { ANYHOW } \\
\text { ANYONE }\end{array}$ & $\begin{array}{r}8 \\
40\end{array}$ & $\begin{array}{l}\text { ARMS } \\
\text { ARMY }\end{array}$ & $\begin{array}{l}2 \\
3\end{array}$ & $\begin{array}{l}\text { ATTEND } \\
\text { ATTENDING }\end{array}$ \\
\hline $\begin{array}{r}196 \\
145 \\
24\end{array}$ & $\begin{array}{l}\text { ANYTHING } \\
\text { ANYWAY } \\
\text { ANYWHERE }\end{array}$ & $\begin{array}{r}65 \\
9 \\
6\end{array}$ & $\begin{array}{l}\text { AROUND } \\
\text { ARRANGE } \\
\text { ARRANGED }\end{array}$ & $\begin{array}{r}4 \\
11 \\
2\end{array}$ & $\begin{array}{l}\text { ATTENTION } \\
\text { ATTITUDE } \\
\text { ATTRACTED }\end{array}$ \\
\hline $\begin{array}{r}24 \\
2 \\
3\end{array}$ & $\begin{array}{l}\text { APART } \\
\text { APARTMENTS } \\
\text { APPALLING }\end{array}$ & $\begin{array}{l}3 \\
5 \\
3\end{array}$ & $\begin{array}{l}\text { ARRANGEMENT } \\
\text { ARRANGEMENTS } \\
\text { ARRANGING }\end{array}$ & $\begin{array}{r}6 \\
8 \\
25\end{array}$ & $\begin{array}{l}\text { ATTRACTIVE } \\
\text { AUDIENCE } \\
\text { AUGUST }\end{array}$ \\
\hline $\begin{array}{r}25 \\
3 \\
3\end{array}$ & $\begin{array}{l}\text { APPARENTLY } \\
\text { APPEAL } \\
\text { APPEALED }\end{array}$ & $\begin{array}{l}2 \\
5 \\
6\end{array}$ & $\begin{array}{l}\text { ARRESTED } \\
\text { ARRIVE } \\
\text { ARRIVED }\end{array}$ & $\begin{array}{l}6 \\
5 \\
2\end{array}$ & $\begin{array}{l}\text { AUNT } \\
\text { AUNTIE } \\
\text { AUSCULTATION }\end{array}$ \\
\hline 2 & APPEALS & 13 & ART & 3 & AUSPICES \\
\hline $\begin{array}{r}13 \\
2\end{array}$ & $\begin{array}{l}\text { APPEAR } \\
\text { APPEARED }\end{array}$ & $\begin{array}{l}3 \\
5\end{array}$ & $\begin{array}{l}\text { ART ICLE } \\
\text { ART ICLES }\end{array}$ & $\begin{array}{l}6 \\
6\end{array}$ & $\begin{array}{l}\text { AUTHOR } \\
\text { AUTHORITIES }\end{array}$ \\
\hline $\begin{array}{l}3 \\
6 \\
3\end{array}$ & $\begin{array}{l}\text { APPEARING } \\
\text { APPEARS } \\
\text { APPLE }\end{array}$ & $\begin{array}{r}2 \\
3 \\
15\end{array}$ & $\begin{array}{l}\text { ARTIFICIAL } \\
\text { ARTIFICI ALLY } \\
\text { ARTS }\end{array}$ & $\begin{array}{r}8 \\
15 \\
6\end{array}$ & $\begin{array}{l}\text { AUTHORITY } \\
\text { AUTHORS } \\
\text { AUTOBIOGRAPHY }\end{array}$ \\
\hline $\begin{array}{r}2 \\
6 \\
17\end{array}$ & $\begin{array}{l}\text { APPLICANT } \\
\text { APPLICANTS } \\
\text { APPLICATION }\end{array}$ & $\begin{array}{r}912 \\
2 \\
3\end{array}$ & $\begin{array}{l}\text { AS } \\
\text { ASH } \\
\text { ASHAMED }\end{array}$ & $\begin{array}{r}2 \\
10 \\
2\end{array}$ & $\begin{array}{l}\text { AUTOMAT ICALLY } \\
\text { AVAILABLE } \\
\text { AVENUE }\end{array}$ \\
\hline $\begin{array}{r}6 \\
23 \\
3\end{array}$ & $\begin{array}{l}\text { APPLICATIONS } \\
\text { APPLIED } \\
\text { APPLIES }\end{array}$ & $\begin{array}{l}2 \\
4 \\
6\end{array}$ & $\begin{array}{l}\text { ASHKEY } \\
\text { ASHTREE } \\
\text { ASIDE }\end{array}$ & $\begin{array}{r}10 \\
5 \\
14\end{array}$ & $\begin{array}{l}\text { AVERAGE } \\
\text { AVOID } \\
\text { AVU }\end{array}$ \\
\hline 16 & APPLY & 57 & ASK & 3 & AWARD \\
\hline $\begin{array}{l}6 \\
2\end{array}$ & $\begin{array}{l}\text { APPLYING } \\
\text { APPOINT }\end{array}$ & $\begin{array}{l}43 \\
18\end{array}$ & $\begin{array}{l}\text { ASKED } \\
\text { ASKING }\end{array}$ & $\begin{array}{l}12 \\
85\end{array}$ & $\begin{array}{l}\text { AWARE } \\
\text { AWAY }\end{array}$ \\
\hline 6 & APPOINTED & 2 & ASKS & 53 & AWEUL \\
\hline $\begin{array}{l}8 \\
3\end{array}$ & $\begin{array}{l}\text { APPOINTMENT } \\
\text { APPOINTMENTS }\end{array}$ & $\begin{array}{l}2 \\
6\end{array}$ & $\begin{array}{l}\text { ASLEEP } \\
\text { ASPECT }\end{array}$ & $\begin{array}{r}22 \\
7\end{array}$ & $\begin{array}{l}\text { AWFULLY } \\
\text { AWKWARD }\end{array}$ \\
\hline 2 & APPRECIATE & 8 & ASPECTS & 16 & $\mathrm{~B}$ \\
\hline $\begin{array}{l}9 \\
6\end{array}$ & $\begin{array}{l}\text { APPROACH } \\
\text { APPROACHED }\end{array}$ & $\begin{array}{l}3 \\
2\end{array}$ & $\begin{array}{l}\text { ASPIRATIONS } \\
\text { ASS }\end{array}$ & $\begin{array}{l}4 \\
3\end{array}$ & $\begin{array}{l}\text { BA } \\
\text { BABIES }\end{array}$ \\
\hline $\begin{array}{l}4 \\
8 \\
2\end{array}$ & $\begin{array}{l}\text { APPROACHING } \\
\text { APPROPRIATE } \\
\text { APPROVE }\end{array}$ & $\begin{array}{l}2 \\
2 \\
5\end{array}$ & $\begin{array}{l}\text { ASSEMBLED } \\
\text { ASSERT } \\
\text { ASSESS }\end{array}$ & $\begin{array}{r}10 \\
221 \\
2\end{array}$ & $\begin{array}{l}\text { BABY } \\
\text { BACK } \\
\text { BACKFORMATION }\end{array}$ \\
\hline $\begin{array}{l}6 \\
2 \\
2\end{array}$ & $\begin{array}{l}\text { APRIL } \\
\text { APRON } \\
\text { ARBITRARY }\end{array}$ & $\begin{array}{r}3 \\
3 \\
20\end{array}$ & $\begin{array}{l}\text { ASSIST } \\
\text { ASSISTANCE } \\
\text { ASSISTANT }\end{array}$ & $\begin{array}{r}11 \\
2 \\
2\end{array}$ & $\begin{array}{l}\text { BACKGROUND } \\
\text { BACKGROUNDS } \\
\text { BACKS }\end{array}$ \\
\hline 2 & ARCADIAN & 2 & ASSISTANTS & 3 & BACKWARDS \\
\hline $\begin{array}{l}3 \\
5\end{array}$ & $\begin{array}{l}\text { ARCHAEOLOGIST } \\
\text { ARCHAEOLOGY }\end{array}$ & $\begin{array}{l}4 \\
2\end{array}$ & $\begin{array}{l}\text { ASSOCIATE } \\
\text { ASSOCIATED }\end{array}$ & $\begin{array}{r}64 \\
9\end{array}$ & $\begin{array}{l}\text { BAD } \\
\text { BADLY }\end{array}$ \\
\hline 2 & ARCHEOLOGY & 6 & ASSUME & 2 & BADTEMPERED \\
\hline $\begin{array}{l}2 \\
2\end{array}$ & $\begin{array}{l}\text { ARCHITECT } \\
\text { ARCH ITECTURE }\end{array}$ & $\begin{array}{l}3 \\
6\end{array}$ & $\begin{array}{l}\text { ASSUMED } \\
\text { ASSUMING }\end{array}$ & $\begin{array}{r}10 \\
6\end{array}$ & $\begin{array}{l}\text { BAG } \\
\text { BAGS }\end{array}$ \\
\hline 816 & ARE & 3 & ASSURE & 4 & BALLET \\
\hline $\begin{array}{r}26 \\
5\end{array}$ & $\begin{array}{l}\text { AREA } \\
\text { AREAS }\end{array}$ & $\begin{array}{l}3 \\
5\end{array}$ & $\begin{array}{l}\text { ASSURED } \\
\text { ASTONISHING }\end{array}$ & $\begin{array}{l}2 \\
2\end{array}$ & $\begin{array}{l}\text { BANANA } \\
\text { BANGING }\end{array}$ \\
\hline 70 & AREN $^{-} \mathrm{T}$ & 2 & ASTOUNDED & 20 & BANK \\
\hline $\begin{array}{r}12 \\
6\end{array}$ & $\begin{array}{l}\text { ARGUE } \\
\text { ARGUMENT }\end{array}$ & $\begin{array}{l}2 \\
2\end{array}$ & $\begin{array}{l}\text { ASTRONOMERS } \\
\text { ASTRONOMY }\end{array}$ & $\begin{array}{l}3 \\
8\end{array}$ & $\begin{array}{l}\text { BANKING } \\
\text { BANKS }\end{array}$ \\
\hline 4 & ARISE & 1031 & $\mathrm{AT}$ & 2 & BANN \\
\hline 2 & ARISES & 4 & ATE & 3 & BAR \\
\hline 4 & ARISTOCRAT & 2 & ATHLETIC & 4 & BARE \\
\hline 2 & ARISTOCRATIC & 4 & ATMOSPHERE & 4 & BARGE \\
\hline $\begin{array}{l}3 \\
5\end{array}$ & $\begin{array}{l}\text { ARM } \\
\text { ARMATURE }\end{array}$ & $\begin{array}{l}2 \\
5\end{array}$ & $\begin{array}{l}\text { ATS } \\
\text { ATTACHED }\end{array}$ & $\begin{array}{l}3 \\
3\end{array}$ & $\begin{array}{l}\text { BARLEYWATER } \\
\text { BARMY }\end{array}$ \\
\hline 2 & ARMCHAIR & 13 & ATTACK & 5 & BARRACKS \\
\hline 3 & ARMED & 7 & ATTEMPT & 2 & BARREL \\
\hline 5 & ARMIES & 2 & ATTEMPTED & 5 & BARRIER \\
\hline 2 & ARMISTICE & 2 & ATTEMPTS & 3 & BASE \\
\hline
\end{tabular}


Appendix A (Continued)

\begin{tabular}{|c|c|c|c|c|c|}
\hline 6 & BASED & 3 & BELT & 2 & BOLUS \\
\hline $\begin{array}{r}2 \\
2 \\
13\end{array}$ & $\begin{array}{l}\text { BASH } \\
\text { BASHING } \\
\text { BASIC }\end{array}$ & $\begin{array}{l}2 \\
3 \\
4\end{array}$ & $\begin{array}{l}\text { BERSERK } \\
\text { BESIDE } \\
\text { BESIDES }\end{array}$ & $\begin{array}{l}4 \\
3 \\
2\end{array}$ & $\begin{array}{l}\text { BOMB } \\
\text { BOMBS } \\
\text { BONE }\end{array}$ \\
\hline 23 & BASICALLY & 2 & BESPOKE & 2 & BONES \\
\hline $\begin{array}{r}2 \\
12\end{array}$ & $\begin{array}{l}\text { BASING } \\
\text { BASIS }\end{array}$ & $\begin{array}{r}55 \\
5\end{array}$ & $\begin{array}{l}\text { BEST } \\
\text { BET }\end{array}$ & $\begin{array}{l}3 \\
3\end{array}$ & $\begin{array}{l}\text { BONNET } \\
\text { BONUS }\end{array}$ \\
\hline 2 & BASKET & 95 & BETTER & 3 & BONY \\
\hline 2 & BASQUE & 81 & BETWEEN & 108 & BOOK \\
\hline 2 & BASQUES & 2 & BEWI LDERED & 2 & BOOKCASE \\
\hline 3 & BASTARD & 2 & BEWILDERING & 3 & BOOKED \\
\hline 8 & ВATCH & 8 & BEYOND & 2 & BOOKING \\
\hline 3 & BATH & 3 & BIBLE & 5 & BOOKLET \\
\hline 5 & BATTALION & 4 & BIBL IOGRAPHICAL & 62 & BOOKS \\
\hline 2 & BATTERY & 16 & BIBLIOGRAPHY & 5 & BOOKSHOP \\
\hline 8 & BATTLE & 75 & $\mathrm{BIG}$ & 8 & BORDER \\
\hline 3 & BATTLES & 11 & BIGGER & 4 & BORE \\
\hline 2 & BATTLING & 2 & BIGGEST & 5 & BORED \\
\hline 2 & BAWD I NESSES & 7 & BILL & 8 & BORING \\
\hline 16 & $\mathrm{BBC}$ & 2 & BI NDAN & 7 & BORN \\
\hline 3 & BBST & 2 & BIOGRAPHICAL & 7 & BORROW \\
\hline 1119 & $\mathrm{BE}$ & 2 & BIOLOGICAL & 6 & BORROWED \\
\hline 2 & BEADING & 2 & BIRDS & 2 & BOTANY \\
\hline 9 & BEAR & 2 & BIRTHDAY & 70 & BOTH \\
\hline 2 & BEARING & 187 & BIT & 17 & BOTHER \\
\hline 40 & BEAUT IFUL & 26 & BITS & 4 & BOTHERED \\
\hline 4 & BEAUT IFULLY & 4 & BITTER & 24 & BOTTLE \\
\hline 2 & BEAUTY & 12 & BLACK & 7 & BOTTLES \\
\hline 9 & BECAME & 4 & BLACKBOARD & 2 & BOTTLING \\
\hline $\begin{array}{r}616 \\
26\end{array}$ & $\begin{array}{l}\text { BECAUSE } \\
\text { BECOME }\end{array}$ & $\begin{array}{l}2 \\
3\end{array}$ & $\begin{array}{l}\text { BLACKLISTED } \\
\text { BLANKET }\end{array}$ & $\begin{array}{r}18 \\
2\end{array}$ & $\begin{array}{l}\text { BOTTOM } \\
\text { BOTTOMS }\end{array}$ \\
\hline 6 & BECOMES & 3 & BLAST ING & 20 & BOUGHT \\
\hline $\begin{array}{r}5 \\
18\end{array}$ & $\begin{array}{l}\text { BECOMING } \\
\text { BED }\end{array}$ & $\begin{array}{l}2 \\
5\end{array}$ & $\begin{array}{l}\text { BLEATS } \\
\text { BLEND }\end{array}$ & $\begin{array}{l}2 \\
7\end{array}$ & $\begin{array}{l}\text { BOUNCE } \\
\text { BOUND }\end{array}$ \\
\hline 2 & BEDEVI LLED & 2 & BLESS & 2 & BOUNDARY \\
\hline 7 & BEDROOM & 5 & BLEW & 4 & BOUT \\
\hline 504 & BEEN & 3 & BLIMEY & 5 & BOW \\
\hline 31 & BEER & 2 & BLIIND & 2 & BOWING \\
\hline 2 & BEERS & 3 & BLITZ & 2 & BOWL \\
\hline 179 & BEFORE & 2 & BLIZZARD & 15 & BOX \\
\hline 6 & BEFOREHAND & 2 & BLOBS & 3 & BOXES \\
\hline $\begin{array}{l}7 \\
9\end{array}$ & $\begin{array}{l}\text { BEG } \\
\text { BEGAN }\end{array}$ & $\begin{array}{l}7 \\
4\end{array}$ & $\begin{array}{l}\text { BLOCK } \\
\text { BLOCKED }\end{array}$ & $\begin{array}{l}15 \\
16\end{array}$ & $\begin{array}{l}\text { BOY } \\
\text { BOYS }\end{array}$ \\
\hline 14 & BEGIN & 12 & BLOKE & 4 & BRACKETS \\
\hline $\begin{array}{r}36 \\
5\end{array}$ & BEGINNING & $\begin{array}{r}7 \\
42\end{array}$ & $\begin{array}{l}\text { BLOOD } \\
\text { BLOODY }\end{array}$ & $\begin{array}{l}7 \\
6\end{array}$ & $\begin{array}{l}\text { BRAIN } \\
\text { BRANCH }\end{array}$ \\
\hline $\begin{array}{l}2 \\
2 \\
2\end{array}$ & $\begin{array}{l}\text { BEGUN } \\
\text { BEHAVE } \\
\text { BEHAVED }\end{array}$ & $\begin{array}{l}2 \\
4 \\
7\end{array}$ & $\begin{array}{l}\text { BLOTTO } \\
\text { BLOW } \\
\text { BLUE }\end{array}$ & $\begin{array}{r}2 \\
2 \\
10\end{array}$ & $\begin{array}{l}\text { BRAND } \\
\text { BRANDY } \\
\text { BREAK }\end{array}$ \\
\hline $\begin{array}{r}5 \\
20 \\
173\end{array}$ & $\begin{array}{l}\text { BEHAVIOUR } \\
\text { BEHIND } \\
\text { BEING }\end{array}$ & $\begin{array}{r}7 \\
3 \\
33\end{array}$ & $\begin{array}{l}\text { BLUFF } \\
\text { BM } \\
\text { BOARD }\end{array}$ & $\begin{array}{l}2 \\
8 \\
5\end{array}$ & $\begin{array}{l}\text { BREAKDOWN } \\
\text { BREAKFAST } \\
\text { BREAKING }\end{array}$ \\
\hline 2 & BELIEF & 8 & BOARDS & 8 & BREAKS \\
\hline 26 & BELIEVE & 9 & BOAT & 2 & BREATHLESS \\
\hline 3 & BELIEVES & 3 & $\mathrm{BOB}$ & 2 & BREATHLESSNESS \\
\hline $\begin{array}{l}6 \\
6 \\
2\end{array}$ & $\begin{array}{l}\text { BELL } \\
\text { BELONG } \\
\text { BELONGING }\end{array}$ & $\begin{array}{r}12 \\
3 \\
5\end{array}$ & $\begin{array}{l}\text { BODY } \\
\text { BOIL } \\
\text { BOILED }\end{array}$ & $\begin{array}{r}11 \\
3 \\
2\end{array}$ & $\begin{array}{l}\text { BREW } \\
\text { BREWERIES } \\
\text { BRICK }\end{array}$ \\
\hline 2 & $\begin{array}{l}\text { BELONGS } \\
\text { BELOW }\end{array}$ & $\begin{array}{l}2 \\
2\end{array}$ & $\begin{array}{l}\text { BOING } \\
\text { BOLD }\end{array}$ & $\begin{array}{l}7 \\
3\end{array}$ & $\begin{array}{l}\text { BRIDGE } \\
\text { BRIEFLY }\end{array}$ \\
\hline
\end{tabular}


Appendix A (Continued)

\begin{tabular}{|c|c|c|c|c|c|}
\hline $\begin{array}{r}15 \\
2 \\
9\end{array}$ & $\begin{array}{l}\text { BRIGHT } \\
\text { BRIGHTER } \\
\text { BRILLIANT }\end{array}$ & $\begin{array}{r}501 \\
4 \\
2\end{array}$ & $\begin{array}{l}\text { CAN } \\
\text { CANAL } \\
\text { CANCEL }\end{array}$ & $\begin{array}{r}87 \\
2 \\
2\end{array}$ & $\begin{array}{l}\text { CERTAINLY } \\
\text { CERT IF ICATE } \\
\text { CHAIN }\end{array}$ \\
\hline $\begin{array}{r}28 \\
8 \\
8\end{array}$ & $\begin{array}{l}\text { BRING } \\
\text { BRINGING } \\
\text { BRINGS }\end{array}$ & $\begin{array}{r}5 \\
11 \\
9\end{array}$ & $\begin{array}{l}\text { CANCER } \\
\text { CANDIDATE } \\
\text { CANDIDATES }\end{array}$ & $\begin{array}{r}10 \\
10 \\
4\end{array}$ & $\begin{array}{l}\text { CHAIR } \\
\text { CHAIRMAN } \\
\text { CHAIRS }\end{array}$ \\
\hline $\begin{array}{l}2 \\
2 \\
2\end{array}$ & $\begin{array}{l}\text { BROAD } \\
\text { BROADER } \\
\text { BROKE }\end{array}$ & $\begin{array}{r}7 \\
300 \\
2\end{array}$ & $\begin{array}{l}\text { CANNOT } \\
\text { CAN }^{\top} T \\
\text { CAPABLE }^{-1}\end{array}$ & $\begin{array}{l}2 \\
2 \\
2\end{array}$ & $\begin{array}{l}\text { CHALLENGING } \\
\text { CHAMELEON } \\
\text { CHAMPAGNE }\end{array}$ \\
\hline $\begin{array}{r}4 \\
10\end{array}$ & $\begin{array}{l}\text { BROKEN } \\
\text { BROT HER }\end{array}$ & $\begin{array}{l}6 \\
8\end{array}$ & $\begin{array}{l}\text { CAPACITY } \\
\text { CAPITAL }\end{array}$ & $\begin{array}{r}14 \\
6\end{array}$ & $\begin{array}{l}\text { CHANCE } \\
\text { CHANCES }\end{array}$ \\
\hline 38 & $\begin{array}{l}\text { BROUGHT } \\
\text { BROWN }\end{array}$ & $\begin{array}{r}4 \\
29\end{array}$ & CAPS & 27 & CHANGE \\
\hline $\begin{array}{l}2 \\
3\end{array}$ & $\begin{array}{l}\text { BROWN } \\
\text { BS }\end{array}$ & $\begin{array}{r}29 \\
2\end{array}$ & CAR & 24 & $\begin{array}{l}\text { CHANGED } \\
\text { CHANGES }\end{array}$ \\
\hline $\begin{array}{l}3 \\
3\end{array}$ & $\begin{array}{l}\text { BS } \\
\text { BUGGER }\end{array}$ & $\begin{array}{l}2 \\
3\end{array}$ & $\begin{array}{l}\text { CARA } \\
\text { CARD }\end{array}$ & $\begin{array}{l}7 \\
6\end{array}$ & $\begin{array}{l}\text { CHANGES } \\
\text { CHANGING }\end{array}$ \\
\hline 4 & BUGGERED & 2 & CARDINAL & 46 & CHAP \\
\hline $\begin{array}{l}4 \\
4\end{array}$ & $\begin{array}{l}\text { BUILD } \\
\text { BUILDERS }\end{array}$ & $\begin{array}{r}7 \\
18\end{array}$ & $\begin{array}{l}\text { CARDS } \\
\text { CARE }\end{array}$ & $\begin{array}{r}2 \\
13\end{array}$ & $\begin{array}{l}\text { CHAPEL } \\
\text { CHAPS }\end{array}$ \\
\hline 29 & BUILDING & 9 & CAREER & 4 & CHAPTER \\
\hline $\begin{array}{r}3 \\
10\end{array}$ & $\begin{array}{l}\text { BUILDINGS } \\
\text { BUILT }\end{array}$ & $\begin{array}{l}7 \\
7\end{array}$ & $\begin{array}{l}\text { CAREFUL } \\
\text { CAREFULLY }\end{array}$ & $\begin{array}{l}4 \\
2\end{array}$ & $\begin{array}{l}\text { CHAPTERS } \\
\text { CHAP S }\end{array}$ \\
\hline $\begin{array}{l}4 \\
2 \\
2\end{array}$ & $\begin{array}{l}\text { BULK } \\
\text { BULLDOZED } \\
\text { BUMMING }\end{array}$ & $\begin{array}{l}2 \\
4 \\
7\end{array}$ & $\begin{array}{l}\text { CAROL } \\
\text { CARRIAGE } \\
\text { CARRIED }\end{array}$ & $\begin{array}{r}29 \\
2 \\
2\end{array}$ & $\begin{array}{l}\text { CHARACTER } \\
\text { CHARACTERISTIC } \\
\text { CHARACTERIZE }\end{array}$ \\
\hline $\begin{array}{l}3 \\
3 \\
3\end{array}$ & $\begin{array}{l}\text { BUMP } \\
\text { BUNCH } \\
\text { BURN }\end{array}$ & $\begin{array}{r}2 \\
2 \\
11\end{array}$ & $\begin{array}{l}\text { CARRIER } \\
\text { CARRIES } \\
\text { CARRY }\end{array}$ & $\begin{array}{l}5 \\
8 \\
2\end{array}$ & $\begin{array}{l}\text { CHARACTERS } \\
\text { CHARGE } \\
\text { CHARGED }\end{array}$ \\
\hline $\begin{array}{l}6 \\
2 \\
6\end{array}$ & $\begin{array}{l}\text { BURNT } \\
\text { BURSARY } \\
\text { BURST }\end{array}$ & $\begin{array}{l}5 \\
4 \\
2\end{array}$ & $\begin{array}{l}\text { CARRYING } \\
\text { CARS } \\
\text { CARTS }\end{array}$ & $\begin{array}{l}2 \\
3 \\
2\end{array}$ & $\begin{array}{l}\text { CHARGES } \\
\text { CHARITY } \\
\text { CHARMER }\end{array}$ \\
\hline $\begin{array}{l}5 \\
4 \\
3\end{array}$ & $\begin{array}{l}\text { BUS } \\
\text { BUSES } \\
\text { BUSH }\end{array}$ & $\begin{array}{r}41 \\
8 \\
3\end{array}$ & $\begin{array}{l}\text { CASE } \\
\text { CASES } \\
\text { CASKS }\end{array}$ & $\begin{array}{l}5 \\
2 \\
5\end{array}$ & $\begin{array}{l}\text { CHARMING } \\
\text { CHASING } \\
\text { CHAT }\end{array}$ \\
\hline 60 & BUSINESS & 9 & CASSETTE & 3 & CHATTERING \\
\hline $\begin{array}{r}14 \\
1652\end{array}$ & $\begin{array}{l}\text { BUSY } \\
\text { BUT }\end{array}$ & $\begin{array}{l}3 \\
4\end{array}$ & $\begin{array}{l}\text { CAST } \\
\text { CASTLE }\end{array}$ & 21 & $\begin{array}{l}\text { CHATT ING } \\
\text { CHEAP }\end{array}$ \\
\hline 2 & BUTTON & 3 & CASUAL & 8 & CHEAPER \\
\hline $\begin{array}{r}2 \\
38\end{array}$ & $\begin{array}{l}\text { BUTTONS } \\
\text { BUY }\end{array}$ & $\begin{array}{l}2 \\
3\end{array}$ & $\begin{array}{l}\text { CASUALLY } \\
\text { CATCH }\end{array}$ & $\begin{array}{l}2 \\
2\end{array}$ & $\begin{array}{l}\text { CHEAPEST } \\
\text { CHEATING }\end{array}$ \\
\hline $\begin{array}{r}10 \\
338 \\
10\end{array}$ & $\begin{array}{l}\text { BUYING } \\
\text { BY } \\
\text { BYE }\end{array}$ & $\begin{array}{l}4 \\
3 \\
7\end{array}$ & $\begin{array}{l}\text { CATEGORIES } \\
\text { CATEGORIZE } \\
\text { CATEGORY }\end{array}$ & $\begin{array}{l}3 \\
3 \\
2\end{array}$ & $\begin{array}{l}\text { CHECK } \\
\text { CHECKER } \\
\text { CHECKING }\end{array}$ \\
\hline 5 & BYPASS & 4 & CATHEDRAL & 2 & CHEERIO \\
\hline $\begin{array}{r}11 \\
5\end{array}$ & $\begin{array}{l}\text { CABLE } \\
\text { CA }\end{array}$ & $\begin{array}{r}13 \\
9\end{array}$ & $\begin{array}{l}\text { CATHOLIC } \\
\text { CATHOLICS }\end{array}$ & $\begin{array}{l}3 \\
7\end{array}$ & $\begin{array}{l}\text { CHEERS } \\
\text { CHEMICAL }\end{array}$ \\
\hline 2 & CAHOOTS & 6 & CAUCASIAN & 6 & CHEMISTRY \\
\hline $\begin{array}{l}2 \\
3\end{array}$ & $\begin{array}{l}\text { CAKE } \\
\text { CALENDAR }\end{array}$ & $\begin{array}{r}12 \\
5\end{array}$ & $\begin{array}{l}\text { CAUGHT } \\
\text { CAUSE }\end{array}$ & $\begin{array}{l}12 \\
15\end{array}$ & $\begin{array}{l}\text { CHERRIES } \\
\text { CHERRY }\end{array}$ \\
\hline 48 & CALL & 6 & CAUSED & 9 & CHIEF \\
\hline $\begin{array}{r}82 \\
4\end{array}$ & $\begin{array}{l}\text { CALLED } \\
\text { CALLING }\end{array}$ & $\begin{array}{l}2 \\
5\end{array}$ & $\begin{array}{l}\text { CAUSEWAY } \\
\text { CAUSING }\end{array}$ & $\begin{array}{l}22 \\
52\end{array}$ & $\begin{array}{l}\text { CHILD } \\
\text { CHILDREN }\end{array}$ \\
\hline 3 & CALLS & 2 & CEILING & 3 & CHILDREN`S \\
\hline $\begin{array}{r}131 \\
2\end{array}$ & $\begin{array}{l}\text { CAME } \\
\text { CAMERA }\end{array}$ & $\begin{array}{r}20 \\
2\end{array}$ & $\begin{array}{l}\text { CENT } \\
\text { CENTRAL }\end{array}$ & $\begin{array}{l}2 \\
2\end{array}$ & $\begin{array}{l}\text { CHILLY } \\
\text { CHIMNEY }\end{array}$ \\
\hline $\begin{array}{l}2 \\
3 \\
3\end{array}$ & $\begin{array}{l}\text { CAMERAS } \\
\text { CAMP } \\
\text { CAMPAIGNING }\end{array}$ & $\begin{array}{r}17 \\
5 \\
3\end{array}$ & $\begin{array}{l}\text { CENTRAL } \\
\text { CENTRE } \\
\text { CENTRES }\end{array}$ & $\begin{array}{r}2 \\
4 \\
13\end{array}$ & $\begin{array}{l}\text { CHINA } \\
\text { CHIVALRY } \\
\text { CHOICE }\end{array}$ \\
\hline $\begin{array}{l}3 \\
2 \\
3\end{array}$ & $\begin{array}{l}\text { CAMP ING } \\
\text { CAMP SITE } \\
\text { CAMPUS }\end{array}$ & $\begin{array}{r}3 \\
36 \\
70\end{array}$ & $\begin{array}{l}\text { CENTURIES } \\
\text { CENTURY } \\
\text { CERTAIN }\end{array}$ & $\begin{array}{l}4 \\
8 \\
2\end{array}$ & $\begin{array}{l}\text { CHOIR } \\
\text { CHOOSE } \\
\text { CHOOSING }\end{array}$ \\
\hline
\end{tabular}


Appendix A (Continued)

\begin{tabular}{|c|c|c|c|c|c|}
\hline $\begin{array}{l}2 \\
2\end{array}$ & $\begin{array}{l}\text { CHOP } \\
\text { CHORES }\end{array}$ & $\begin{array}{r}3 \\
10\end{array}$ & $\begin{array}{l}\text { COAL } \\
\text { COAST }\end{array}$ & $\begin{array}{l}5 \\
4\end{array}$ & $\begin{array}{l}\text { COMP ARED } \\
\text { COMP ARI SON }\end{array}$ \\
\hline $\begin{array}{l}4 \\
6 \\
8\end{array}$ & $\begin{array}{l}\text { CHOSE } \\
\text { CHOSEN } \\
\text { CHRIST }\end{array}$ & $\begin{array}{l}5 \\
4 \\
3\end{array}$ & $\begin{array}{l}\text { COAT } \\
\text { COBBLES } \\
\text { COCK }\end{array}$ & $\begin{array}{l}2 \\
8 \\
6\end{array}$ & $\begin{array}{l}\text { COMPENSATION } \\
\text { COMPETENT } \\
\text { COMP ETITION }\end{array}$ \\
\hline $\begin{array}{r}32 \\
3 \\
2\end{array}$ & $\begin{array}{l}\text { CHRISTMAS } \\
\text { CHUCKED } \\
\text { CHUNK }\end{array}$ & $\begin{array}{r}2 \\
44 \\
32\end{array}$ & $\begin{array}{l}\text { COCKTAIL } \\
\text { COFFEE } \\
\text { COLD }\end{array}$ & $\begin{array}{l}2 \\
2 \\
2\end{array}$ & $\begin{array}{l}\text { COMPET IT IVE } \\
\text { COMPETITORS } \\
\text { COMP I LE }\end{array}$ \\
\hline 10 & CHURCH & 6 & COLLABORATION & 6 & COMP LAIN \\
\hline $\begin{array}{l}2 \\
4\end{array}$ & $\begin{array}{l}\text { CIG } \\
\text { CIGARETTE }\end{array}$ & $\begin{array}{l}2 \\
2\end{array}$ & $\begin{array}{l}\text { COLLAPSE } \\
\text { COLLAPSING }\end{array}$ & $\begin{array}{l}5 \\
5\end{array}$ & $\begin{array}{l}\text { COMP LAINED } \\
\text { COMPLAINING }\end{array}$ \\
\hline 2 & CIGARLETTE & 2 & COLLEAGUE & 7 & COMPLAINS \\
\hline 2 & CIGGIE & 7 & COLLEAGUES & 19 & COMPLETE \\
\hline 2 & CINEMA & 3 & COLLECT & 35 & COMP LETELY \\
\hline 4 & CINEMAS & 3 & COLLECTED & 13 & COMPLICATED \\
\hline $\begin{array}{l}5 \\
4\end{array}$ & $\begin{array}{l}\text { CIRCLE } \\
\text { CIRCLES }\end{array}$ & $\begin{array}{l}2 \\
2\end{array}$ & $\begin{array}{l}\text { COLLECTION } \\
\text { COLLECTIVE }\end{array}$ & $\begin{array}{l}2 \\
3\end{array}$ & $\begin{array}{l}\text { COMPLICATION } \\
\text { COMPLICATIONS }\end{array}$ \\
\hline 2 & CIRCUITS & 79 & COLLEGE & 11 & COMPREHENSION \\
\hline $\begin{array}{r}8 \\
12\end{array}$ & $\begin{array}{l}\text { CIRCULAR } \\
\text { CIRCUMSTANCE }\end{array}$ & $\begin{array}{l}9 \\
2\end{array}$ & $\begin{array}{l}\text { COLLEGES } \\
\text { COLLEGIATE }\end{array}$ & $\begin{array}{l}2 \\
2\end{array}$ & $\begin{array}{l}\text { COMPREHENSIVE } \\
\text { COMPROMISE }\end{array}$ \\
\hline 8 & CIRCUMSTANCES & 16 & COLOUR & 2 & COMPULSORY \\
\hline 2 & CITIZEN & 2 & COLOURED & 14 & COMP UTER \\
\hline 6 & CITY & 6 & COLOURS & 2 & COMPUTERIZED \\
\hline $\begin{array}{l}8 \\
2\end{array}$ & $\begin{array}{l}\text { CIVIL } \\
\text { CIVILIAN }\end{array}$ & $\begin{array}{l}3 \\
8\end{array}$ & $\begin{array}{l}\text { COLUMN } \\
\text { COMBINATION }\end{array}$ & $\begin{array}{l}7 \\
2\end{array}$ & $\begin{array}{l}\text { COMPUTERS } \\
\text { COMPUT ING }\end{array}$ \\
\hline 2 & CIVILIANS & 2 & COMBINE & 2 & CONCEIVABLE \\
\hline $\begin{array}{l}3 \\
4 \\
3\end{array}$ & $\begin{array}{l}\text { CIVILIZATION } \\
\text { CLAIM } \\
\text { CLAIMS }\end{array}$ & $\begin{array}{r}2 \\
261 \\
50\end{array}$ & $\begin{array}{l}\text { COMBINED } \\
\text { COME } \\
\text { COMES }\end{array}$ & $\begin{array}{l}3 \\
2 \\
5\end{array}$ & $\begin{array}{l}\text { CONCEIVED } \\
\text { CONCENTRATED } \\
\text { CONCERN }\end{array}$ \\
\hline 2 & CLASH & 5 & COMFORT & 22 & CONCERNED \\
\hline $\begin{array}{l}13 \\
20\end{array}$ & $\begin{array}{l}\text { CLASS } \\
\text { CLASSES }\end{array}$ & $\begin{array}{l}6 \\
2\end{array}$ & $\begin{array}{l}\text { COMFORTABLE } \\
\text { COMFORTABLY }\end{array}$ & $\begin{array}{l}4 \\
4\end{array}$ & $\begin{array}{l}\text { CONCERNS } \\
\text { CONCERT }\end{array}$ \\
\hline 2 & CLASSIC & 74 & COMING & 9 & CONCLUSION \\
\hline $\begin{array}{l}4 \\
7\end{array}$ & $\begin{array}{l}\text { CLASSICAL } \\
\text { CLASSICS }\end{array}$ & $\begin{array}{l}5 \\
3\end{array}$ & $\begin{array}{l}\text { COMMAND } \\
\text { COMMANDERINCHIEE }\end{array}$ & $\begin{array}{l}2 \\
2\end{array}$ & $\begin{array}{l}\text { CONCLUSIONS } \\
\text { CONCURRED }\end{array}$ \\
\hline 3 & CLAUSE & 2 & COMMANDING & 4 & CONDEMN \\
\hline 2 & CLEAN & 14 & COMMENT & 6 & CONDITION \\
\hline 25 & CLEAR & 2 & COMMENTED & 6 & CONDITIONS \\
\hline 5 & CLEARED & 5 & COMMENTS & 5 & CONDUCT \\
\hline 3 & CLEARER & 7 & COMMISSION & 2 & CONDUCTED \\
\hline 12 & CLEARLY & 2 & COMMIT & 3 & CONDUCTOR \\
\hline 11 & CLEVER & 3 & COMMITMENT & 8 & CONF ERENCE \\
\hline 2 & CLEVERLY & 5 & COMMITTED & $\begin{array}{l}5 \\
2\end{array}$ & $\begin{array}{l}\text { CONF ERENCES } \\
\text { CONF IDENCE }\end{array}$ \\
\hline 2 & CLICK & 18 & $\begin{array}{l}\text { COMMITTEE } \\
\text { COMMON }\end{array}$ & 2 & \\
\hline 4 & CLIENT & 13 & COMMON & 3 & \\
\hline 6 & CLIENTS & 17 & COMMONROOM & 2 & CONFUSED \\
\hline 2 & CLIENT S & 2 & COMMUNAL & 2 & CONFUSING \\
\hline 7 & CLIMATE & 2 & COMMUNICATE & 3 & CONFUSION \\
\hline $\begin{array}{r}3 \\
18\end{array}$ & $\begin{array}{l}\text { CLINICAL } \\
\text { CLOSE }\end{array}$ & $\begin{array}{r}2 \\
12\end{array}$ & $\begin{array}{l}\text { COMMUNICATING } \\
\text { COMMUNICATION }\end{array}$ & $\begin{array}{l}5 \\
2\end{array}$ & $\begin{array}{l}\text { CONGRESS } \\
\text { CONNECT }\end{array}$ \\
\hline 3 & CLOSED & 5 & COMMUNICATIONS & 6 & CONNECTED \\
\hline $\begin{array}{l}5 \\
4\end{array}$ & $\begin{array}{l}\text { CLOSELY } \\
\text { CLOSER }\end{array}$ & $\begin{array}{l}6 \\
3\end{array}$ & $\begin{array}{l}\text { COMMUNIST } \\
\text { COMMUNITY }\end{array}$ & $\begin{array}{l}8 \\
4\end{array}$ & $\begin{array}{l}\text { CONNECTION } \\
\text { CONNECTIONS }\end{array}$ \\
\hline 3 & CLOSET & 3 & COMMUTE & 2 & CONNEXION \\
\hline $\begin{array}{l}2 \\
3\end{array}$ & $\begin{array}{l}\text { CLOTHES } \\
\text { CLOUDS }\end{array}$ & $\begin{array}{l}2 \\
5\end{array}$ & $\begin{array}{l}\text { COMMUTING } \\
\text { COMP ANIES }\end{array}$ & $\begin{array}{l}4 \\
3\end{array}$ & $\begin{array}{l}\text { CONSCIOUS } \\
\text { CONSEQUENTLY }\end{array}$ \\
\hline 4 & CLUB & 29 & COMPANY & 4 & CONSERVATIVE \\
\hline 2 & CLUE & 2 & COMP ANY'S & 12 & CONSIDER \\
\hline 3 & CLUSTER & 3 & COMP ARAT IVE & 2 & CONSIDERABLE \\
\hline 2 & COACHING & 4 & COMPARE & 2 & CONSIDERATION \\
\hline
\end{tabular}


Appendix A (Continued)

\begin{tabular}{|c|c|c|c|c|c|}
\hline 4 & CONSIDERATIONS & 2 & CORRELATIONS & 3 & CROWDED \\
\hline 10 & CONSIDERED & 4 & CORRELATIVE & 5 & CROWN \\
\hline 3 & CONSIDERING & 5 & CORRESPONDENCE & 3 & CRUNCH \\
\hline 4 & CONSISTENTLY & 2 & CORRESPONDING & 4 & $\csc$ \\
\hline 6 & CONSTABULARY & 11 & CORRIDOR & 2 & CUBE \\
\hline 3 & CONSTANTLY & 200 & $\cos$ & 3 & CUDDLY \\
\hline 2 & CONSTRAINTS & 14 & $\operatorname{cosT}$ & 2 & CULTURAL \\
\hline 2 & CONSTRUCTION & 7 & COSTS & 2 & CULTURE \\
\hline 2 & CONSULT & 2 & COTTAGE & 2 & CUM \\
\hline 2 & CONSULTANCY & 322 & COULD & 2 & CUMULAT IVE \\
\hline 4 & CONSULTANT & 120 & COULDN`T & 9 & CUP \\
\hline $\begin{array}{l}2 \\
4\end{array}$ & $\begin{array}{l}\text { CONSULTANTS } \\
\text { CONSULTED }\end{array}$ & $\begin{array}{r}25 \\
9\end{array}$ & $\begin{array}{l}\text { COUNCIL } \\
\text { COUNT }\end{array}$ & $\begin{array}{l}5 \\
9\end{array}$ & $\begin{array}{l}\text { CUPBOARD } \\
\text { CUPS }\end{array}$ \\
\hline 11 & CONTACT & 5 & COUNTER & 3 & CURE \\
\hline 2 & CONTACTED & 2 & COUNTING & 10 & CURIOUS \\
\hline 5 & CONTACTS & 5 & COUNTRIES & 3 & CURIOUSLY \\
\hline 2 & CONTAIN & 50 & COUNTRY & 2 & CURRENT \\
\hline 4 & CONTAINS & 2 & COUNTS & 3 & CURR ICULUM \\
\hline 2 & CONTEMPORARIES & 14 & COUNTY & 2 & CURRY \\
\hline 5 & CONTENT & 61 & COUPLE & 2 & CURTAINS \\
\hline 2 & CONTENTS & 2 & COUPLED & 2 & CURVE \\
\hline 4 & CONTEXT & 295 & COURSE & 2 & CUSTOMER \\
\hline 2 & CONT INUALLY & 3 & COURSEBOOK & 34 & CUT \\
\hline 3 & CONT INUATION & 15 & COURSES & 6 & CUTT ING \\
\hline 4 & CONT INUE & 14 & COURT & 2 & CYCLE \\
\hline 5 & CONT INUOUS & 5 & COUSIN & 3 & CYNICAL \\
\hline 2 & CONTRACEPTIVE & 2 & COVENANT & 2 & CYPHERS \\
\hline 3 & CONTRACT & 9 & COVER & 9 & CYST \\
\hline 6 & CONTRAST & 2 & COVERAGE & 4 & CYSTS \\
\hline 3 & CONTRIBUTE & 13 & COVERED & 13 & D \\
\hline 11 & CONTRIBUTION & 2 & COWS & 3 & DADDY \\
\hline 11 & CONTROL & 2 & CRACK & 2 & DAGGER \\
\hline 2 & CONTROLLER & 2. & CRANE & 5 & DAGGERS \\
\hline 7 & CONVENIENT & 2 & CRAZE & 4 & DAILY \\
\hline 3 & CONVENT IONAL & 4 & CRAZY & 2 & DAMAGE \\
\hline 3 & CONVENTIONS & 2 & CREAM & 4 & DAMMIT \\
\hline 18 & CONVERSATION & 5 & CREAMY & 11 & DAMN \\
\hline 2 & CONVERSION & 5 & CREATE & 5 & DAMNED \\
\hline 2 & CONVERTED & 4 & CREATED & 4 & DANCE \\
\hline 2 & CONVEX & 3 & CREATION & 3 & DANCING \\
\hline 3 & CONVINCE & 2 & CREATURE & 3 & DANGER \\
\hline 7 & CONVINCED & 2 & CREDIBLE & 2 & DARE \\
\hline 2 & CONVINCING & 2 & CREDIT & 2 & DAREN`T \\
\hline 6 & COOKING & 2 & CRICKET & 13 & DARK \\
\hline 3 & COOPERATE & 4 & CRIKEY & 26 & DARL ING \\
\hline 2 & COOPERATION & 4 & CRIME & 11 & DATE \\
\hline 2 & COORDINATE & 2 & CRITERIA & 7 & DAUGHTER \\
\hline 2 & COORDINATES & 5 & CRITERION & 113 & DAY \\
\hline 4 & COORDINATING & 4 & CRITIC & 62 & DAYS \\
\hline 9 & COPE & 6 & CRITICAL & 2 & DAZZLING \\
\hline 3 & COPIED & 3 & CRITICALLY & 8 & DEAD \\
\hline 9 & COPIES & 15 & CRITICISM & 2 & DEADLY \\
\hline 26 & COPY & 2 & CRITICIZE & 29 & DEAL \\
\hline $\begin{array}{r}10 \\
4\end{array}$ & $\begin{array}{l}\text { COR } \\
\text { CORE }\end{array}$ & $\begin{array}{l}4 \\
2\end{array}$ & $\begin{array}{l}\text { CRITICS } \\
\text { CROPS }\end{array}$ & $\begin{array}{r}14 \\
3\end{array}$ & $\begin{array}{l}\text { DEAL ING } \\
\text { DEALT }\end{array}$ \\
\hline 20 & CORNER & 23 & CROSS & 51 & DEAR \\
\hline 3 & CORPORAL & 3 & CROSSES & 15 & DEATH \\
\hline 4 & CORRECT & 3 & CROSSING & 2 & DEBATABLE \\
\hline 4 & CORRECTLY & 2 & CROSSINGS & 2 & DEBT \\
\hline 2 & CORRELATION & 3 & CROWD & 2 & DECADES \\
\hline
\end{tabular}


Appendix A (Continued)

\begin{tabular}{|c|c|c|c|c|c|}
\hline $\begin{array}{r}5 \\
3 \\
19\end{array}$ & $\begin{array}{l}\text { DECEMBER } \\
\text { DECENT } \\
\text { DECIDE }\end{array}$ & $\begin{array}{l}2 \\
2 \\
8\end{array}$ & $\begin{array}{l}\text { DESCR IBES } \\
\text { DESCRIBING } \\
\text { DESCRIPT ION }\end{array}$ & $\begin{array}{r}6 \\
8 \\
10\end{array}$ & $\begin{array}{l}\text { DIRT } \\
\text { DIRTY } \\
\text { DISABLED }\end{array}$ \\
\hline 29 & DECIDED & 2 & DESERVED & 3 & DISADVANTAGES \\
\hline $\begin{array}{r}2 \\
10\end{array}$ & $\begin{array}{l}\text { DECIDING } \\
\text { DECISION }\end{array}$ & $\begin{array}{l}2 \\
2\end{array}$ & $\begin{array}{l}\text { DESERVES } \\
\text { DESIGN }\end{array}$ & $\begin{array}{l}3 \\
6\end{array}$ & $\begin{array}{l}\text { DI SAPPEAR } \\
\text { DISAPPEARED }\end{array}$ \\
\hline $\begin{array}{l}2 \\
3 \\
2\end{array}$ & $\begin{array}{l}\text { DECISIVELY } \\
\text { DECLARED } \\
\text { DECLINE }\end{array}$ & $\begin{array}{l}9 \\
2 \\
7\end{array}$ & $\begin{array}{l}\text { DESIGNED } \\
\text { DESIRABLE } \\
\text { DESK }\end{array}$ & $\begin{array}{l}2 \\
5 \\
3\end{array}$ & $\begin{array}{l}\text { DISAPPEARING } \\
\text { DISAPPOINTED } \\
\text { DISAPPOINTMENT }\end{array}$ \\
\hline $\begin{array}{l}2 \\
2 \\
2\end{array}$ & $\begin{array}{l}\text { DECODE } \\
\text { DECORATED } \\
\text { DECORATING }\end{array}$ & $\begin{array}{l}2 \\
2 \\
7\end{array}$ & $\begin{array}{l}\text { DESPAIR } \\
\text { DESPERATE } \\
\text { DESPERATELY }\end{array}$ & $\begin{array}{l}5 \\
5 \\
3\end{array}$ & $\begin{array}{l}\text { DISAPPROVE } \\
\text { DISASTER } \\
\text { DISASTROUS }\end{array}$ \\
\hline $\begin{array}{l}2 \\
2 \\
4\end{array}$ & $\begin{array}{l}\text { DECORATION } \\
\text { DECORATIONS } \\
\text { DEDICATED }\end{array}$ & $\begin{array}{r}2 \\
2 \\
14\end{array}$ & $\begin{array}{l}\text { DESPITE } \\
\text { DESTROYED } \\
\text { DETAIL }\end{array}$ & $\begin{array}{l}2 \\
2 \\
2\end{array}$ & $\begin{array}{l}\text { DISC } \\
\text { DISCIPIINE } \\
\text { DISCIPLINES }\end{array}$ \\
\hline $\begin{array}{l}2 \\
2 \\
6\end{array}$ & $\begin{array}{l}\text { DEDUCING } \\
\text { DEEMED } \\
\text { DEEP }\end{array}$ & $\begin{array}{l}5 \\
3 \\
3\end{array}$ & $\begin{array}{l}\text { DETAILED } \\
\text { DETAILS } \\
\text { DETECT }\end{array}$ & $\begin{array}{r}14 \\
2 \\
9\end{array}$ & $\begin{array}{l}\text { DISCOVERED } \\
\text { DISCS } \\
\text { DISCUSS }\end{array}$ \\
\hline 7 & DEEPER & 5 & DETERM INED & 4 & DISCUSSED \\
\hline $\begin{array}{l}2 \\
4\end{array}$ & $\begin{array}{l}\text { DEEPLY } \\
\text { DEFEATED }\end{array}$ & $\begin{array}{l}2 \\
4\end{array}$ & $\begin{array}{l}\text { DEVALUED } \\
\text { DEVELOP }\end{array}$ & $\begin{array}{l}2 \\
8\end{array}$ & $\begin{array}{l}\text { DI SCUSSING } \\
\text { DISCUSSION }\end{array}$ \\
\hline 2 & DEFEATING & 6 & DEVELOPED & 2 & DISCUSSIONS \\
\hline 3 & DEFEND & 2 & DEVELOPING & 13 & DISEASE \\
\hline 2 & DEFENSIVE & 8 & DEVELOPMENT & 2 & DISHEVELLED \\
\hline $\begin{array}{r}2 \\
6 \\
23\end{array}$ & $\begin{array}{l}\text { DEF INE } \\
\text { DEF INITE } \\
\text { DEF INITELY }\end{array}$ & $\begin{array}{l}2 \\
2 \\
6\end{array}$ & $\begin{array}{l}\text { DEVELOPS } \\
\text { DEVICE } \\
\text { DEVIL }\end{array}$ & $\begin{array}{l}6 \\
5 \\
4\end{array}$ & $\begin{array}{l}\text { DISLIKE } \\
\text { DISLIKED } \\
\text { DISPARATES }\end{array}$ \\
\hline $\begin{array}{r}2 \\
34 \\
10\end{array}$ & $\begin{array}{l}\text { DEFINITION } \\
\text { DEGREE } \\
\text { DEGREES }\end{array}$ & $\begin{array}{l}2 \\
2 \\
4\end{array}$ & $\begin{array}{l}\text { DEVOTED } \\
\text { DEVOTING } \\
\text { DHOBI }\end{array}$ & $\begin{array}{l}2 \\
2 \\
2\end{array}$ & $\begin{array}{l}\text { DISPARITY } \\
\text { DISPENSE } \\
\text { DISPOSAL }\end{array}$ \\
\hline $\begin{array}{l}3 \\
2 \\
4\end{array}$ & $\begin{array}{l}\text { DELAY } \\
\text { DELAYED } \\
\text { DELIBERATELY }\end{array}$ & $\begin{array}{l}5 \\
2 \\
2\end{array}$ & $\begin{array}{l}\text { DIAGNOSIS } \\
\text { DIALECTS } \\
\text { DIARISTS }\end{array}$ & $\begin{array}{l}2 \\
3 \\
2\end{array}$ & $\begin{array}{l}\text { DISPOSED } \\
\text { DISSERTATION } \\
\text { DISSOLVE }\end{array}$ \\
\hline $\begin{array}{l}2 \\
2 \\
2\end{array}$ & $\begin{array}{l}\text { DELIGHTED } \\
\text { DELIGHTFUL } \\
\text { DELIVER }\end{array}$ & $\begin{array}{r}7 \\
20 \\
14\end{array}$ & $\begin{array}{l}\text { DIARRHOEA } \\
\text { DIARY } \\
\text { DICTIONARY }\end{array}$ & $\begin{array}{l}8 \\
4 \\
8\end{array}$ & $\begin{array}{l}\text { DISTANCE } \\
\text { DISTINCT } \\
\text { DISTINCTION }\end{array}$ \\
\hline 4 & DELIVERED & 412 & DID & 4 & DISTINCTIVE \\
\hline $\begin{array}{l}2 \\
4\end{array}$ & $\begin{array}{l}\text { DELIVERY } \\
\text { DEMAND }\end{array}$ & $\begin{array}{r}304 \\
3\end{array}$ & $\begin{array}{l}\text { DIDN'T }^{\prime} \\
\text { DIE }\end{array}$ & $\begin{array}{l}6 \\
3\end{array}$ & $\begin{array}{l}\text { DI ST INGUISH } \\
\text { DI ST INGUISHED }\end{array}$ \\
\hline $\begin{array}{l}3 \\
2 \\
3\end{array}$ & $\begin{array}{l}\text { DEMANDS } \\
\text { DEMOLISHED } \\
\text { DEMONSTRATED }\end{array}$ & $\begin{array}{l}15 \\
24 \\
90\end{array}$ & $\begin{array}{l}\text { DIED } \\
\text { DIFFERENCE } \\
\text { DIFFERENT }\end{array}$ & $\begin{array}{l}2 \\
7 \\
3\end{array}$ & $\begin{array}{l}\text { DI STORTION } \\
\text { DISTRICT } \\
\text { DISTURBING }\end{array}$ \\
\hline $\begin{array}{r}10 \\
9 \\
5\end{array}$ & $\begin{array}{l}\text { DENTAL } \\
\text { DENT IST } \\
\text { DENTISTS }\end{array}$ & $\begin{array}{r}4 \\
63 \\
8\end{array}$ & $\begin{array}{l}\text { DIFFERENTLY } \\
\text { DIFF ICULT } \\
\text { DIFF ICULTIES }\end{array}$ & $\begin{array}{l}2 \\
2 \\
3\end{array}$ & $\begin{array}{l}\text { DIVE } \\
\text { DIVERTED } \\
\text { DIVIDE }\end{array}$ \\
\hline 2 & DENY & 19 & DIFF ICULTY & 6 & DIVISION \\
\hline $\begin{array}{r}2 \\
105\end{array}$ & $\begin{array}{l}\text { DEPART } \\
\text { DEPARTMENT }\end{array}$ & $\begin{array}{l}2 \\
6\end{array}$ & $\begin{array}{l}\text { DIGESTED } \\
\text { DIGGING }\end{array}$ & $\begin{array}{r}6 \\
1060\end{array}$ & $\begin{array}{l}\text { DIVISIONS } \\
\text { DO }\end{array}$ \\
\hline 15 & DEPARTMENTAL & 27 & DINNER & 32 & DOCTOR \\
\hline $\begin{array}{r}15 \\
2\end{array}$ & $\begin{array}{l}\text { DEPARTMENTS } \\
\text { DEPEND }\end{array}$ & $\begin{array}{l}2 \\
5\end{array}$ & $\begin{array}{l}\text { DI PLOMA } \\
\text { DIPLOMATIC }\end{array}$ & $\begin{array}{l}2 \\
4\end{array}$ & $\begin{array}{l}\text { DOCTRINE } \\
\text { DOCUMENT }\end{array}$ \\
\hline $\begin{array}{l}9 \\
4 \\
2\end{array}$ & $\begin{array}{l}\text { DEPENDS } \\
\text { DEPLORABLE } \\
\text { DEPRESSED }\end{array}$ & $\begin{array}{l}3 \\
5 \\
3\end{array}$ & $\begin{array}{l}\text { DIRE } \\
\text { DIRECT } \\
\text { DIRECTED }\end{array}$ & $\begin{array}{r}2 \\
3 \\
220\end{array}$ & $\begin{array}{l}\text { DOCUMENTATION } \\
\text { DOCUMENTS } \\
\text { DOES }\end{array}$ \\
\hline $\begin{array}{l}3 \\
3 \\
2\end{array}$ & $\begin{array}{l}\text { DEPTH } \\
\text { DEPTHS } \\
\text { DER }\end{array}$ & $\begin{array}{l}2 \\
8 \\
4\end{array}$ & $\begin{array}{l}\text { DIRECTING } \\
\text { DIRECTION } \\
\text { DIRECTIONS }\end{array}$ & $\begin{array}{r}152 \\
8 \\
4\end{array}$ & $\begin{array}{l}\text { DOESN'T } \\
\text { DOG } \\
\text { DOGS }\end{array}$ \\
\hline 2 & DESCENDING & 3 & DIRECTLY & 218 & DOING \\
\hline $\begin{array}{l}5 \\
4\end{array}$ & $\begin{array}{l}\text { DESCRIBE } \\
\text { DESCRIBED }\end{array}$ & $\begin{array}{r}16 \\
4\end{array}$ & $\begin{array}{l}\text { DIRECTOR } \\
\text { DIRECTORS }\end{array}$ & $\begin{array}{l}4 \\
4\end{array}$ & $\begin{array}{l}\text { DOLE } \\
\text { DOLLARS }\end{array}$ \\
\hline
\end{tabular}


Appendix A (Continued)

\begin{tabular}{|c|c|c|c|c|c|}
\hline $\begin{array}{l}2 \\
6\end{array}$ & $\begin{array}{l}\text { DOME } \\
\text { DOMEST IC }\end{array}$ & $\begin{array}{l}8 \\
7\end{array}$ & $\begin{array}{l}\text { DRUGS } \\
\text { DRUNK }\end{array}$ & $\begin{array}{r}12 \\
6\end{array}$ & $\begin{array}{l}\text { EIGHTEENTH } \\
\text { EIGHTH }\end{array}$ \\
\hline $\begin{array}{r}2 \\
2 \\
182\end{array}$ & $\begin{array}{l}\text { DOMINATE } \\
\text { DON } \\
\text { DONE }\end{array}$ & $\begin{array}{l}2 \\
3 \\
5\end{array}$ & $\begin{array}{l}\text { DRUNKEN } \\
\text { DRY } \\
\text { DU }\end{array}$ & $\begin{array}{r}3 \\
2 \\
14\end{array}$ & $\begin{array}{l}\text { EIGHTHS } \\
\text { EIGHTIES } \\
\text { EIGHTY }\end{array}$ \\
\hline $\begin{array}{r}2 \\
3 \\
906\end{array}$ & $\begin{array}{l}\text { DONKEY S } \\
\text { DONNE } \\
\text { DON`T }\end{array}$ & $\begin{array}{l}4 \\
2 \\
8\end{array}$ & $\begin{array}{l}\text { DUBIOUS } \\
\text { DUD } \\
\text { DUE }\end{array}$ & $\begin{array}{r}3 \\
5 \frac{1}{6}\end{array}$ & $\begin{array}{l}\text { EIGHTYEIGHT } \\
\text { EITHER } \\
\text { ELABORATE }\end{array}$ \\
\hline $\begin{array}{r}25 \\
4 \\
2\end{array}$ & $\begin{array}{l}\text { DOOR } \\
\text { DOORS } \\
\text { DOSSIER }\end{array}$ & $\begin{array}{l}2 \\
5 \\
3\end{array}$ & $\begin{array}{l}\text { DUG } \\
\text { DULL } \\
\text { DUPLICATE }\end{array}$ & $\begin{array}{r}3 \\
10 \\
4\end{array}$ & $\begin{array}{l}\text { ELECTION } \\
\text { ELECTRIC } \\
\text { ELECTRICAL }\end{array}$ \\
\hline $\begin{array}{r}3 \\
2 \\
16\end{array}$ & $\begin{array}{l}\text { DOT } \\
\text { DOTTED } \\
\text { DOUBLE }\end{array}$ & $\begin{array}{r}38 \\
5 \\
8\end{array}$ & $\begin{array}{l}\text { DURING } \\
\text { DUST } \\
\text { DUTIES }\end{array}$ & $\begin{array}{l}2 \\
2 \\
9\end{array}$ & $\begin{array}{l}\text { ELEMENT } \\
\text { ELEMENTARY } \\
\text { ELEVEN }\end{array}$ \\
\hline $\begin{array}{r}18 \\
2 \\
229\end{array}$ & $\begin{array}{l}\text { DOUBT } \\
\text { DOUBTFUL } \\
\text { DOWN }\end{array}$ & $\begin{array}{l}2 \\
3 \\
8\end{array}$ & $\begin{array}{l}\text { DUTY } \\
\text { DYING } \\
\text { D'YOU }\end{array}$ & $\begin{array}{l}3 \\
2 \\
5\end{array}$ & $\begin{array}{l}\text { ELICITED } \\
\text { ELICITS } \\
\text { ELITE }\end{array}$ \\
\hline $\begin{array}{l}2 \\
3 \\
4\end{array}$ & $\begin{array}{l}\text { DOWNFALL } \\
\text { DOWN STAIRS } \\
\text { DOZEN }\end{array}$ & $\begin{array}{r}12 \\
65 \\
3\end{array}$ & $\begin{array}{l}\text { E } \\
\text { EACH } \\
\text { EARL }\end{array}$ & $\begin{array}{r}104 \\
4 \\
2\end{array}$ & $\begin{array}{l}\text { ELSE } \\
\text { ELSEWHERE } \\
\text { EMBARK }\end{array}$ \\
\hline 4 & DRAFT & 18 & EARLIER & 4 & EMBARRASSED \\
\hline 2 & DRAFTED & 34 & EARLY & $\begin{array}{l}8 \\
5\end{array}$ & EMBARRASSING \\
\hline $\begin{array}{l}4 \\
6\end{array}$ & $\begin{array}{l}\text { DRAFT ING } \\
\text { DRAIN }\end{array}$ & 6 & $\begin{array}{l}\text { EARN } \\
\text { EAPNFCTIY }\end{array}$ & $\begin{array}{l}5 \\
6\end{array}$ & EMERGENCIES \\
\hline $\begin{array}{r}6 \\
3 \\
25\end{array}$ & $\begin{array}{l}\text { DRAIN } \\
\text { DRAINAGE } \\
\text { DRAMA }\end{array}$ & $\begin{array}{l}2 \\
4 \\
2\end{array}$ & $\begin{array}{l}\text { EARNESTLY } \\
\text { EARNING } \\
\text { EARS }\end{array}$ & $\begin{array}{l}6 \\
2 \\
3\end{array}$ & $\begin{array}{l}\text { EMERGENCY } \\
\text { EMINENTLY } \\
\text { EMOT ION }\end{array}$ \\
\hline $\begin{array}{l}8 \\
2 \\
2\end{array}$ & $\begin{array}{l}\text { DRAMATIC } \\
\text { DRAMAT IST } \\
\text { DRAUGHT }\end{array}$ & $\begin{array}{r}14 \\
2 \\
18\end{array}$ & $\begin{array}{l}\text { EARTH } \\
\text { EARTHY } \\
\text { EASIER }\end{array}$ & $\begin{array}{l}2 \\
6 \\
3\end{array}$ & $\begin{array}{l}\text { EMOT IONAL } \\
\text { EMP HASIS } \\
\text { EMP IRE }\end{array}$ \\
\hline $\begin{array}{r}10 \\
2 \\
2\end{array}$ & $\begin{array}{l}\text { DRAW } \\
\text { DRAWBACK } \\
\text { DRAWER }\end{array}$ & $\begin{array}{r}14 \\
17 \\
3\end{array}$ & $\begin{array}{l}\text { EASILY } \\
\text { EAST } \\
\text { EASTER }\end{array}$ & $\begin{array}{l}2 \\
2 \\
6\end{array}$ & $\begin{array}{l}\text { EMP LOY } \\
\text { EMP LOYED } \\
\text { EMPTY }\end{array}$ \\
\hline $\begin{array}{l}3 \\
2 \\
2\end{array}$ & $\begin{array}{l}\text { DRAWING } \\
\text { DRAWINGS } \\
\text { DRAWN }\end{array}$ & $\begin{array}{r}3 \\
21 \\
9\end{array}$ & $\begin{array}{l}\text { EASTERN } \\
\text { EASY } \\
\text { EAT }\end{array}$ & $\begin{array}{l}2 \\
3 \\
2\end{array}$ & $\begin{array}{l}\text { EN } \\
\text { ENABLE } \\
\text { ENCLOSED }\end{array}$ \\
\hline $\begin{array}{l}2 \\
8 \\
2\end{array}$ & $\begin{array}{l}\text { DRAWS } \\
\text { DREADFUL } \\
\text { DREADFULLY }\end{array}$ & $\begin{array}{l}7 \\
6 \\
4\end{array}$ & $\begin{array}{l}\text { EATING } \\
\text { ECONOMIC } \\
\text { ECONOMICS }\end{array}$ & $\begin{array}{l}2 \\
5 \\
2\end{array}$ & $\begin{array}{l}\text { ENCOUNTER } \\
\text { ENCOURAGE } \\
\text { ENCOURAGEMENT }\end{array}$ \\
\hline 3 & DREAM & 2 & ECONOMY & 116 & END \\
\hline $\begin{array}{l}2 \\
3\end{array}$ & $\begin{array}{l}\text { DREAMS } \\
\text { DREARY }\end{array}$ & $\begin{array}{l}5 \\
2\end{array}$ & $\begin{array}{l}\text { EDGE } \\
\text { EDITED }\end{array}$ & $1 \frac{1}{3}$ & $\begin{array}{l}\text { ENDED } \\
\text { ENDI NG }\end{array}$ \\
\hline 3 & DREGS & 3 & EDITING & 3 & ENDLESS \\
\hline $\begin{array}{l}2 \\
4\end{array}$ & $\begin{array}{l}\text { DREW } \\
\text { DRIFT }\end{array}$ & $\begin{array}{l}3 \\
4\end{array}$ & $\begin{array}{l}\text { EDITION } \\
\text { EDITIONS }\end{array}$ & $\begin{array}{l}7 \\
2\end{array}$ & $\begin{array}{l}\text { ENDS } \\
\text { ENEMA }\end{array}$ \\
\hline $\begin{array}{r}2 \\
3 \\
25\end{array}$ & $\begin{array}{l}\text { DRILL } \\
\text { DRILLS } \\
\text { DRINK }\end{array}$ & $\begin{array}{l}3 \\
2 \\
3\end{array}$ & $\begin{array}{l}\text { EDITOR } \\
\text { EDITORIAL } \\
\text { EDUCATED }\end{array}$ & $\begin{array}{l}2 \\
4 \\
6\end{array}$ & $\begin{array}{l}\text { ENEMAS } \\
\text { ENERGY } \\
\text { ENGAGED }\end{array}$ \\
\hline $\begin{array}{l}2 \\
6 \\
2\end{array}$ & $\begin{array}{l}\text { DRINKING } \\
\text { DRINKS } \\
\text { DRIP }\end{array}$ & $\begin{array}{r}18 \\
6 \\
11\end{array}$ & $\begin{array}{l}\text { EDUCATION } \\
\text { EDUCAT IONAL } \\
\text { EFFECT }\end{array}$ & $\begin{array}{l}6 \\
5 \\
2\end{array}$ & $\begin{array}{l}\text { ENGI NE } \\
\text { ENGI NEER ING } \\
\text { ENG I NEERS }\end{array}$ \\
\hline 20 & DRIVE & 3 & EFFECTIVE & 2 & ENGRAVED \\
\hline $\begin{array}{l}7 \\
2\end{array}$ & $\begin{array}{l}\text { DRIVEN } \\
\text { DRIVER }\end{array}$ & $\begin{array}{l}3 \\
4\end{array}$ & $\begin{array}{l}\text { EFFECTIVELY } \\
\text { EFFICIENT }\end{array}$ & $\begin{array}{l}9 \\
8\end{array}$ & $\begin{array}{l}\text { ENJOY } \\
\text { ENJOYED }\end{array}$ \\
\hline 4 & DRIVES & 3 & EFFICIENTLY & 2 & ENJOYING \\
\hline $\begin{array}{r}16 \\
9\end{array}$ & $\begin{array}{l}\text { DRIVING } \\
\text { DROP }\end{array}$ & $\begin{array}{l}6 \\
6\end{array}$ & $\begin{array}{l}\text { EFFORT } \\
\text { EGG }\end{array}$ & $\begin{array}{r}2 \\
17\end{array}$ & $\begin{array}{l}\text { ENLARGER } \\
\text { ENORMOUS }\end{array}$ \\
\hline 9 & DROPPED & 7 & EGGS & 3 & ENORMOUSLY \\
\hline 2 & $\begin{array}{l}\text { DROPPING } \\
\text { DROVE }\end{array}$ & 6 & EH & 106 & ENOUGH \\
\hline 5 & $\begin{array}{l}\text { DRUVE } \\
\text { DRUG }\end{array}$ & $\begin{array}{l}38 \\
21\end{array}$ & $\begin{array}{l}\text { EIGHT } \\
\text { EIGHTEEN }\end{array}$ & $\begin{array}{l}4 \\
3\end{array}$ & $\begin{array}{l}\text { ENTER } \\
\text { ENTERTAINED }\end{array}$ \\
\hline
\end{tabular}


Appendix A (Continued)

\begin{tabular}{|c|c|c|c|c|c|}
\hline 2 & ENTERTAINING & 4 & EXAMP LES & 2 & EXTENSIVE \\
\hline $\begin{array}{l}2 \\
5 \\
5\end{array}$ & $\begin{array}{l}\text { ENTERTAINMENT } \\
\text { ENTHUSIASTIC } \\
\text { ENTIRE }\end{array}$ & $\begin{array}{l}6 \\
2 \\
4\end{array}$ & $\begin{array}{l}\text { EXAMS } \\
\text { EXASPERATING } \\
\text { EXCEEDINGLY }\end{array}$ & $\begin{array}{r}20 \\
9 \\
11\end{array}$ & $\begin{array}{l}\text { EXTENT } \\
\text { EXTERNAL } \\
\text { EXTRA }\end{array}$ \\
\hline $\begin{array}{r}29 \\
2\end{array}$ & $\begin{array}{l}\text { ENT IRELY } \\
\text { ENURETIC }\end{array}$ & $\begin{array}{r}9 \\
45\end{array}$ & $\begin{array}{l}\text { EXCELLENT } \\
\text { EXCEPT }\end{array}$ & $\begin{array}{r}2 \\
12\end{array}$ & $\begin{array}{l}\text { EXTRAMURAL } \\
\text { EXTRAORDINARY }\end{array}$ \\
\hline 3 & ENVI RONMENT & 2 & EXCEPTION & 28 & EXTREMELY \\
\hline 2 & ENVISAGED & 2 & EXCEPTIONAL & 12 & EYE \\
\hline 2 & EPISODE & 4 & EXCESSIVE & 19 & EYES \\
\hline 3 & EQUAL & 5 & EXCHANGE & 7 & \\
\hline $\begin{array}{r}10 \\
3\end{array}$ & $\begin{array}{l}\text { EQUALLY } \\
\text { EQUALS }\end{array}$ & $\begin{array}{l}2 \\
4\end{array}$ & $\begin{array}{l}\text { EXCITED } \\
\text { EXCITING }\end{array}$ & $\begin{array}{r}4 \\
25\end{array}$ & $\begin{array}{l}\text { FABULOUS } \\
\text { FACE }\end{array}$ \\
\hline 6 & EQUIPMENT & 2 & EXCLUDED & 2 & FACED \\
\hline 4 & EQU IPPED & 3 & EXCLUSIVELY & 3 & FACILITIES \\
\hline 5 & EQUITY & 2 & EXCRUCIAT ING & 193 & FACT \\
\hline 11 & EQUIVALENT & 9 & EXCUSE & 4 & FACTOR \\
\hline 2 & ERODED & 4 & EXECUT IVE & 2 & FACTORS \\
\hline $\begin{array}{l}2 \\
4\end{array}$ & $\begin{array}{l}\text { ERROR } \\
\text { ESCORT }\end{array}$ & $\begin{array}{l}7 \\
2\end{array}$ & $\begin{array}{l}\text { EXERCISE } \\
\text { EXERCISED }\end{array}$ & $\begin{array}{l}4 \\
2\end{array}$ & $\begin{array}{l}\text { FACTS } \\
\text { FACTUAL }\end{array}$ \\
\hline 13 & ESPECIALLY & 2 & EXHAUSTING & 21 & FACULTY \\
\hline 2 & ESPIONAGE & 2 & EXHAUSTION & 4 & FAIL \\
\hline 10 & ESSAY & 2 & EXHIBITS & 8 & FAILED \\
\hline 7 & ESSAYS & 5 & EXIST & 5 & FAILURE \\
\hline 2 & ESSENTIAL & 3 & EXISTED & 4 & FAINT \\
\hline 4 & ESSENT IALLY & 6 & EXISTENCE & 25 & FAIR \\
\hline 7 & ESTABLISHED & 17 & EXISTING & 2 & FAIRIES \\
\hline 4 & ESTABLISHMENT & 2 & EXISTS & 41 & FAIRLY \\
\hline 4 & ESTATE & 3 & EXPANSION & 7 & FAITH \\
\hline 3 & $\mathrm{ET}$ & 32 & EXPECT & 7 & FALL \\
\hline $\begin{array}{r}15 \\
2\end{array}$ & $\begin{array}{l}\text { ETCETERA } \\
\text { ETIOLOGY }\end{array}$ & $\begin{array}{l}2 \\
8\end{array}$ & $\begin{array}{l}\text { EXPECTANT } \\
\text { EXPECTED }\end{array}$ & $\begin{array}{l}2 \\
4\end{array}$ & $\begin{array}{l}\text { FALSE } \\
\text { FAME }\end{array}$ \\
\hline 2 & EUPHEMISM & 3 & EXPECTING & 6 & FAMILIES \\
\hline 135 & EVEN & 5 & EXPECTS & 31 & FAMILY \\
\hline 32 & EVENING & 2 & EXPEDITION & 2 & FAMOUS \\
\hline 9 & EVENINGS & 2 & EXPENDITURE & 2 & FAN \\
\hline 7 & EVENT & 5 & EXPENSE & 8 & FANCY \\
\hline 4 & EVENTS & 3 & EXPENSES & 16 & FANTASTIC \\
\hline 10 & EVENTUALLY & 34 & EXPENSIVE & 2 & FANTASY \\
\hline 99 & EVER & 19 & EXPERIENCE & 101 & FAR \\
\hline 80 & EVERY & 2 & EXPERIENCED & 5 & FARE \\
\hline 68 & EVERYBODY & 2 & EXPERIMENT & 2 & FARES \\
\hline 7 & $\begin{array}{l}\text { EVERYBODY S } \\
\text { EVERYONE }\end{array}$ & $\begin{array}{l}6 \\
2\end{array}$ & $\begin{array}{l}\text { EXPERIMENTAL } \\
\text { EXPERIMENTS }\end{array}$ & $\begin{array}{l}5 \\
5\end{array}$ & $\begin{array}{l}\text { FARM } \\
\text { FARMER }\end{array}$ \\
\hline $\begin{array}{l}18 \\
74\end{array}$ & $\begin{array}{l}\text { EVEKYUNE } \\
\text { EVERYTHING }\end{array}$ & 10 & EXPERT & 2 & $\begin{array}{l}\text { FARMER } \\
\text { FASCICLE }\end{array}$ \\
\hline $\begin{array}{r}74 \\
5\end{array}$ & EVERYWHERE & 4 & EXPERTS & 2 & FASCINATED \\
\hline 4 & EVIDENCE & 13 & EXPLAIN & 10 & FASCINATING \\
\hline 3 & EVIDENT & 5 & EXPLAINED & 3 & FASHION \\
\hline 4 & EXACT & 2 & EXPLAINING & 3 & FASHIONABLE \\
\hline 62 & EXACTLY & 2 & EXPLANATORY & 12 & FAST \\
\hline 3 & EXAGGERATED & 3 & EXPLODE & $\begin{array}{l}3 \\
5\end{array}$ & FASTER \\
\hline 2 & EXAGGERATION & 2 & EXPLORE & 5 & FAT \\
\hline 11 & EXAM & 2 & EXPORT & 4 & FATAL \\
\hline 12 & EXAMINATION & 5 & EXPOSED & 2 & FATE \\
\hline 4 & EXAMINATIONS & 4 & EXPRESS & 46 & F ATHER \\
\hline 5 & EXAMINE & 2 & EXPRESSES & 13 & FATHER'S \\
\hline 2 & EXAMINED & 2 & EXPRESSING & 8 & FAULT \\
\hline 3 & EXAMINER & 6 & EXPRESSION & 2 & FAULTS \\
\hline 5 & EXAMINERS & 4 & EXPRESSIONIST & 4 & FAVOUR \\
\hline 9 & EXAMINING & 2 & EXTEND & 7 & FAVOURITE \\
\hline 42 & EXAMP LE & 2 & EXTENSION & 7 & FEAR \\
\hline
\end{tabular}


Appendix A (Continued)

\begin{tabular}{|c|c|c|c|c|c|}
\hline 2 & FEATURE & 49 & FINE & 2 & FOOLE்D \\
\hline 6 & FEATURES & 6 & FINGER & 2 & FOOLISH \\
\hline 3 & FEBRUARY & 3 & F INGERS & 3 & FOOLS \\
\hline 8 & FED & 19 & FINISH & 10 & FOOT \\
\hline 4 & FEED & 35 & FINISHED & 2 & FOOT ING \\
\hline 100 & FEEL & 6 & FINISHING & 1157 & \\
\hline 30 & FEEL ING & 15 & FIRE & 4 & FORCE \\
\hline 7 & FEEL INGS & 2 & FIREBREAK & 4 & FORCED \\
\hline 12 & FEELS & 2 & FIREF IGHTING & 2 & FORCES \\
\hline 23 & FEET & 7 & FIRES & 9 & FOREIGN \\
\hline 3 & FELL & 19 & FIRM & 2 & FOREST \\
\hline 15 & FELLOW & 3 & FIRMLY & 2 & FORESTRY \\
\hline 3 & FELLOWS & 7 & FIRMS & 3 & FOREVER \\
\hline 7 & FELLOWSHIP & 183 & FIRST & 17 & FORGET \\
\hline 3 & FELLOWSHIPS & 3 & FIRSTLY & 4 & FORGETTING \\
\hline 36 & FELT & 2 & FISHING & 2 & FORGIVEN \\
\hline 4 & FEMALE & 16 & FIT & 5 & FORGOT \\
\hline 4 & FERRET & 2 & FITS & 13 & FORGOTTEN \\
\hline 4 & FERRY & 2 & FITTED & 25 & FORM \\
\hline 8 & FESTIVAL & 106 & FIVE & 3 & FORMAL \\
\hline 73 & FEW & 2 & FIVEPENCE & 2 & FORMALLY \\
\hline 4 & FICTION & 10 & FIXED & 3 & FORMED \\
\hline 2 & FIDDLE & 2 & FLAKE & 5 & FORMS \\
\hline 2 & FIDDLED & 41 & FLAT & 3 & FORMULA \\
\hline 7 & FIDDLING & 2 & FLATMATE & 10 & FORTH \\
\hline 28 & FIELD & 5 & FLATS & 10 & FORTNIGHT \\
\hline 6 & FIELDS & 2 & FLAUT IST & 6 & FORTUNATELY \\
\hline 2 & FIENDS & 2 & FLESH & 3 & FORTUNE \\
\hline 31 & FIFTEEN & 3 & ELEW & 16 & FORTY \\
\hline 7 & FIFTEENTH & 2 & FLEXIBILITY & 3 & FORTYFIVE \\
\hline 6 & FIFTH & 3 & FLICK & 2 & FORTYNINE \\
\hline 3 & FIFT IES & 2 & FLICKED & 2 & FORTYTWO \\
\hline 41 & FIFTY & 2 & FLICKKNIEE & 17 & FORWARD \\
\hline 5 & FIFTYFIVE & 6 & FLIES & 2 & FOSSILIZED \\
\hline 4 & F IFTYFOUR & 6 & FLOAT ING & 4 & FOUGHT \\
\hline 5 & FIFTYONE & 2 & FLOGGED & 2 & FOUL \\
\hline 2 & FIFTYSEVEN & 3 & FLOOD & 2 & FOULES \\
\hline 8 & FIGHT & 6 & F LOOR & 70 & FOUND \\
\hline 5 & FIGHTING & 2 & FLOP & 13 & FOUNDATION \\
\hline 9 & F IGURE' & 2 & FLOPPY & 2 & FOUNDED \\
\hline 4 & FIGURES & 2 & FLOUR & 108 & FOUR \\
\hline 4 & FILE & 3 & FLOWERS & 7 & FOURTEEN \\
\hline 4 & FILING & 2 & FLUCTUATING & 7 & FOURTEENTH \\
\hline 7 & FILL & 2 & FLUENCY & 10 & FOURTH \\
\hline 4 & FILLED & 2 & FLUENT & 3 & FOX \\
\hline 3 & FILLING & 2 & $F\llcorner Y$ & 4 & FRANCS \\
\hline 45 & F ILM & 2 & FLYING & 4 & FRANK \\
\hline 8 & FILMS & 7 & FOCUS & 5 & FRANKLY \\
\hline 3 & FILTHY & 2 & FOLD & 2 & FRAUD \\
\hline 2 & FINAL & 3 & FOLDED & 26 & FREE \\
\hline 6 & FINALISTS & 2 & FOLK & 2 & FREED \\
\hline 11 & FINALLY & 8 & FOLKLORE & 3 & FREELANCE \\
\hline 10 & FINALS & 15 & FOLLOW & 2 & FREQUENT \\
\hline 2 & FINANCED & 10 & FOLLOWED & 6 & FREQUENTLY \\
\hline 2 & FINANCES & 15 & FOLLOWING & 4 & FRESH \\
\hline 7 & FINANCIAL & 4 & FOLLOWS & 11 & FRIDAY \\
\hline 3 & FINANCIALLY & 3 & FOLLY & 23 & FRIEND \\
\hline 145 & FIND & 5 & FOND & 7 & FRIENDLY \\
\hline 8 & FINDING & 12 & FOOD & 29 & FRIENDS \\
\hline 3 & FINDS & 7 & FOOL & 2 & FRIGHTENING \\
\hline
\end{tabular}


Appendix A (Continued)

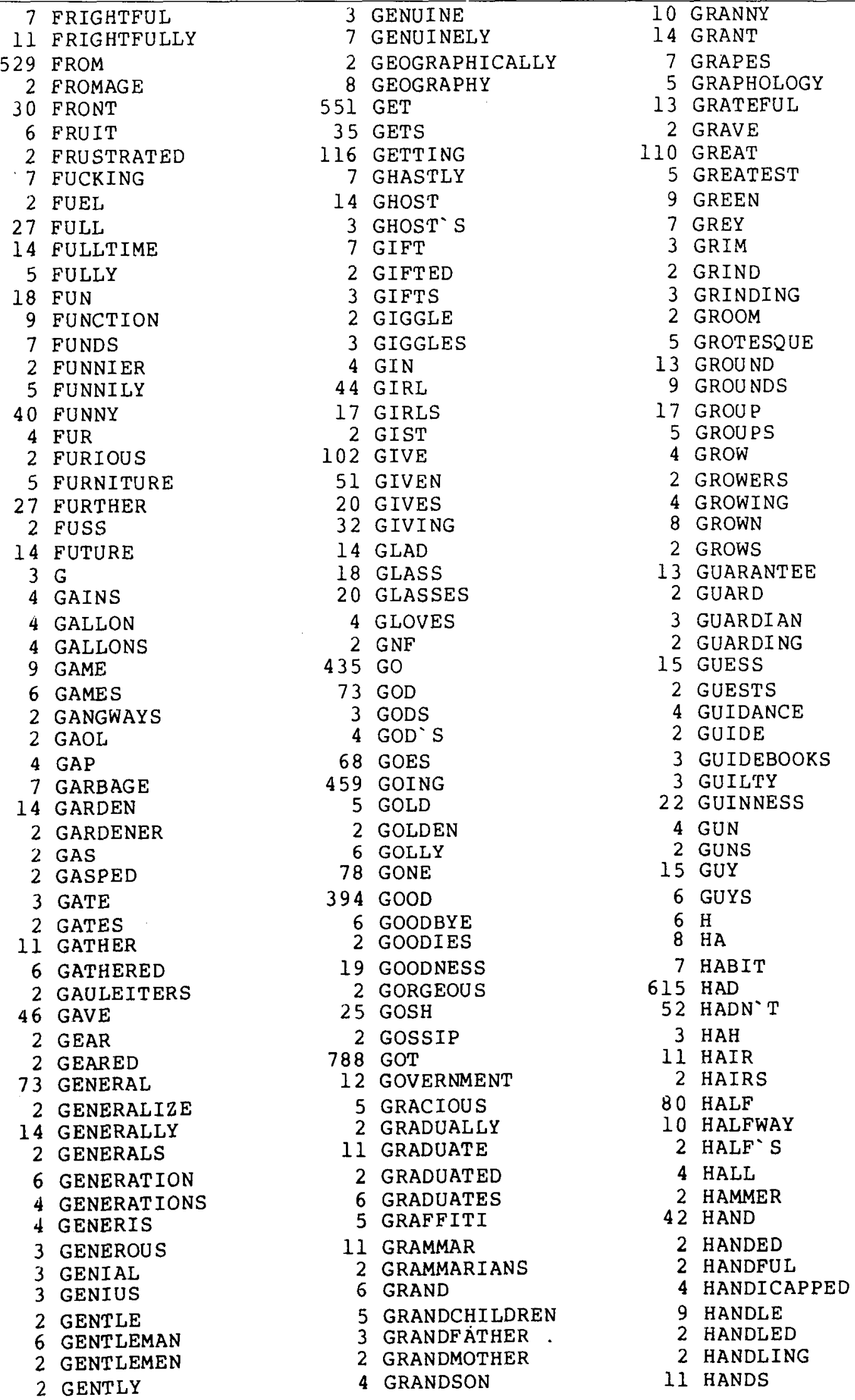


Appendix A (Continued)

\begin{tabular}{|c|c|c|c|c|c|}
\hline 3 & HANDWRITING & 3 & HESITATION & 3 & HOU SEMOTHER \\
\hline 4 & HANDY & 2 & HEY & 5 & HOUSES \\
\hline $\begin{array}{l}8 \\
4\end{array}$ & $\begin{array}{l}\text { HANG } \\
\text { HANGING }\end{array}$ & $\begin{array}{r}2 \\
59\end{array}$ & $\begin{array}{l}\text { HEYDAY } \\
\text { HE'D }\end{array}$ & $\begin{array}{r}329 \\
15\end{array}$ & $\begin{array}{l}\text { HOW } \\
\text { HOWEVER }\end{array}$ \\
\hline $\begin{array}{l}41 \\
56 \\
10\end{array}$ & $\begin{array}{l}\text { HAPPEN } \\
\text { HAPPENED } \\
\text { HAPPENING }\end{array}$ & $\begin{array}{r}20 \\
393 \\
2\end{array}$ & $\begin{array}{l}\text { HE'LL } \\
\text { HE S } \\
\text { HIDDEN }\end{array}$ & $\begin{array}{r}7 \\
12 \\
5\end{array}$ & $\begin{array}{l}\text { HOW S } \\
\text { HUGE } \\
\text { HUH }\end{array}$ \\
\hline 22 & HAPPENS & 2 & HIDE & 11 & HUMAN \\
\hline $\begin{array}{l}3 \\
4\end{array}$ & $\begin{array}{l}\text { HAPPIER } \\
\text { HAPPILY }\end{array}$ & $\begin{array}{l}4 \\
6\end{array}$ & $\begin{array}{l}\text { HIDEOUS } \\
\text { HIERARCHY }\end{array}$ & $\begin{array}{r}4 \\
84\end{array}$ & $\begin{array}{l}\text { HUMOUR } \\
\text { HUNDRED }\end{array}$ \\
\hline $\begin{array}{r}32 \\
2 \\
2\end{array}$ & $\begin{array}{l}\text { HAPPY } \\
\text { HARANGUE } \\
\text { HARBOUR }\end{array}$ & $\begin{array}{l}29 \\
13 \\
17\end{array}$ & $\begin{array}{l}\text { HIGH } \\
\text { HIGHER } \\
\text { HIGHLY }\end{array}$ & $\begin{array}{l}4 \\
2 \\
4\end{array}$ & $\begin{array}{l}\text { HUNDREDS } \\
\text { HUNG } \\
\text { HURRY }\end{array}$ \\
\hline $\begin{array}{r}32 \\
6 \\
9\end{array}$ & $\begin{array}{l}\text { HARD } \\
\text { HARDER } \\
\text { HARDLY }\end{array}$ & $\begin{array}{l}3 \\
5 \\
2\end{array}$ & $\begin{array}{l}\text { HIGHPOWERED } \\
\text { HILLS } \\
\text { HILL'S }\end{array}$ & $\begin{array}{r}4 \\
14 \\
3\end{array}$ & $\begin{array}{l}\text { HURT } \\
\text { HUSBAND } \\
\text { HYDRAHEADED }\end{array}$ \\
\hline $\begin{array}{r}3 \\
6 \\
313\end{array}$ & $\begin{array}{l}\text { HARM } \\
\text { HARMFUL } \\
\text { HAS }\end{array}$ & $\begin{array}{r}362 \\
35 \\
3\end{array}$ & $\begin{array}{l}\text { HIM } \\
\text { HIMSELF } \\
\text { HINT }\end{array}$ & $\begin{array}{r}3 \\
4 \\
6797\end{array}$ & $\begin{array}{l}\text { HYDROLOGY } \\
\text { HYSTERICS } \\
\text { I }\end{array}$ \\
\hline 50 & HASN $T$ & 2 & HINTS & 57 & IDEA \\
\hline $\begin{array}{l}3 \\
9\end{array}$ & $\begin{array}{l}\text { HAT } \\
\text { HATE }\end{array}$ & $\begin{array}{l}4 \\
2\end{array}$ & $\begin{array}{l}\text { HIRE } \\
\text { HIRED }\end{array}$ & $\begin{array}{l}5 \\
2\end{array}$ & $\begin{array}{l}\text { IDEAL } \\
\text { IDEALISM }\end{array}$ \\
\hline $\begin{array}{r}3 \\
2 \\
1365\end{array}$ & $\begin{array}{l}\text { HATES } \\
\text { HATS } \\
\text { HAVE }\end{array}$ & $\begin{array}{r}354 \\
6 \\
2\end{array}$ & $\begin{array}{l}\text { HIS } \\
\text { HISTORICAL } \\
\text { HISTORIES }\end{array}$ & $\begin{array}{r}2 \\
15 \\
2\end{array}$ & $\begin{array}{l}\text { IDEALLY } \\
\text { IDEAS } \\
\text { IDENTIFY }\end{array}$ \\
\hline $\begin{array}{r}187 \\
119 \\
1464\end{array}$ & $\begin{array}{l}\text { HAVEN }{ }^{\circ} \\
\text { HAVING } \\
\text { HE }\end{array}$ & $\begin{array}{r}51 \\
10 \\
3\end{array}$ & $\begin{array}{l}\text { HISTORY } \\
\text { HIT } \\
\text { HO }\end{array}$ & $\begin{array}{l}2 \\
2 \\
2\end{array}$ & $\begin{array}{l}\text { IDIOM } \\
\text { IDIOMS } \\
\text { IDIOSYNCRATIC }\end{array}$ \\
\hline 45 & HEAD & 18 & HOLD & 2 & IDIOTS \\
\hline $\begin{array}{l}3 \\
2\end{array}$ & $\begin{array}{l}\text { HEADACHE } \\
\text { HEADLAND }\end{array}$ & $\begin{array}{r}7 \\
19\end{array}$ & $\begin{array}{l}\text { HOLDING } \\
\text { HOLIDAY }\end{array}$ & $\begin{array}{r}4 \\
757\end{array}$ & $\begin{array}{l}\text { IE } \\
\text { IF }\end{array}$ \\
\hline 3 & HEADMASTER & 9 & HOLIDAYS & 4 & IGNORANCE \\
\hline 9 & HEADS & 3 & HOLY & 2 & IGNORANT \\
\hline 10 & HEALTH & 81 & HOME & 12 & ILL \\
\hline $\begin{array}{r}34 \\
82 \\
2\end{array}$ & $\begin{array}{l}\text { HEAR } \\
\text { HEARD } \\
\text { HEARING }\end{array}$ & $\begin{array}{r}2 \\
5 \\
11\end{array}$ & $\begin{array}{l}\text { HOMES } \\
\text { HONEST } \\
\text { HONESTLY }\end{array}$ & $\begin{array}{l}3 \\
2 \\
3\end{array}$ & $\begin{array}{l}\text { ILLEGAL } \\
\text { ILLEGIBLE } \\
\text { ILLOGICAL }\end{array}$ \\
\hline 14 & HEART & 3 & HONEYMOON & 2 & ILLTREATED \\
\hline 2 & HEAT & 24 & HONOURS & 2 & ILLUMINATED \\
\hline 8 & HEAT ING & 2 & HOOK & 10 & IMAGE \\
\hline 2 & HEAVEN & 34 & HOPE & 2 & IMAGERY \\
\hline 13 & HEAVENS & 2 & HOPED & 6 & IMAG INATION \\
\hline 11 & HEAVILY & 4 & HOPELESS & 29 & IMAG INE \\
\hline 7 & HEAVY & 4 & HOPES & 3 & IMAG INED \\
\hline $\begin{array}{r}4 \\
10\end{array}$ & $\begin{array}{l}\text { HECTIC } \\
\text { HELD }\end{array}$ & $\begin{array}{l}7 \\
2\end{array}$ & $\begin{array}{l}\text { HOPING } \\
\text { HOPS }\end{array}$ & $\begin{array}{l}2 \\
5\end{array}$ & $\begin{array}{l}\text { IMMATURE } \\
\text { IMMEDIATE }\end{array}$ \\
\hline 29 & HELL & 2 & HORIZON & 25 & IMMEDI ATELY \\
\hline 16 & HELLO & 4 & HORNY & 2 & IMMENSE \\
\hline 43 & HELP & 20 & HORR IB LE & 3 & IMPACT \\
\hline 5 & HELPED & 2 & HORRIBLY & 2 & IMP ERF ECT \\
\hline 9 & HELPFUL & 4 & HORRID & 3 & IMPERIAL \\
\hline 5 & HELP ING & 4 & HORR IF IED & 2 & IMPORT \\
\hline $\begin{array}{l}2 \\
3\end{array}$ & $\begin{array}{l}\text { HELPLESS } \\
\text { HELPS }\end{array}$ & $\begin{array}{r}4 \\
12\end{array}$ & $\begin{array}{l}\text { HORRORS } \\
\text { HOSPITAL }\end{array}$ & $\begin{array}{r}3 \\
35\end{array}$ & $\begin{array}{l}\text { IMPORTANCE } \\
\text { IMPORTANT }\end{array}$ \\
\hline 8 & HENCE & 7 & HOT & 3 & IMPOSED \\
\hline 255 & HER & 9 & HOTEL & 2 & IMPOSING \\
\hline 216 & HERE & 27 & HOUR & 14 & IMPOSSIBLE \\
\hline 5 & HERE` $S$ & 27 & HOURS & 2 & IMPOSSIBLY \\
\hline 2 & HERS & 57 & HOUSE & 2 & IMP RACT ICAB LE \\
\hline 8 & HERSELF & 3 & HOUSEHOLD & 2 & IMPRESS \\
\hline
\end{tabular}


Appendix A (Continued)

\begin{tabular}{|c|c|c|c|c|c|}
\hline $\begin{array}{r}3 \\
18 \\
2\end{array}$ & $\begin{array}{l}\text { IMPRESSED } \\
\text { IMPRESSION } \\
\text { IMPRESSIONIST }\end{array}$ & $\begin{array}{l}2 \\
6 \\
5\end{array}$ & $\begin{array}{l}\text { INSIST } \\
\text { INSISTED } \\
\text { INSPECTOR }\end{array}$ & $\begin{array}{r}17 \\
2100 \\
3\end{array}$ & $\begin{array}{l}\text { IRA } \\
\text { IS } \\
\text { ISLAND }\end{array}$ \\
\hline $\begin{array}{l}8 \\
5 \\
4\end{array}$ & $\begin{array}{l}\text { IMPRESSIONS } \\
\text { IMPRESSIVE } \\
\text { IMPROVE }\end{array}$ & $\begin{array}{r}2 \\
2 \\
41\end{array}$ & $\begin{array}{l}\text { INSP IRED } \\
\text { INSTALMENTS } \\
\text { INS'TANCE }\end{array}$ & $\begin{array}{r}257 \\
2 \\
2\end{array}$ & $\begin{array}{l}\text { ISN`T } \\
\text { ISOLATED } \\
\text { ISOLATION }\end{array}$ \\
\hline $\begin{array}{l}2 \\
2\end{array}$ & $\begin{array}{l}\text { IMPROVED } \\
\text { IMP ROVEMENT }\end{array}$ & $\begin{array}{r}2 \\
23\end{array}$ & $\begin{array}{l}\text { INSTANT } \\
\text { INSTEAD }\end{array}$ & $\begin{array}{l}5 \\
3\end{array}$ & $\begin{array}{l}\text { ISSUE } \\
\text { ISSUED }\end{array}$ \\
\hline 2 & IMPROVES & 2 & INST INCT & 3653 & IT \\
\hline $\begin{array}{r}2 \\
3123 \\
2\end{array}$ & $\begin{array}{l}\text { IMPROVISED } \\
\text { IN } \\
\text { INBREEDING }\end{array}$ & $\begin{array}{r}8 \\
5 \\
10\end{array}$ & $\begin{array}{l}\text { INST ITUTE } \\
\text { INST ITUTION } \\
\text { INSTITUTIONS }\end{array}$ & $\begin{array}{l}2 \\
2 \\
5\end{array}$ & $\begin{array}{l}\text { ITA } \\
\text { ITEM } \\
\text { ITEMS }\end{array}$ \\
\hline $\begin{array}{l}2 \\
2 \\
8\end{array}$ & $\begin{array}{l}\text { INCAPAB LE } \\
\text { INCENT IVE } \\
\text { INCIDENTALLY }\end{array}$ & $\begin{array}{l}4 \\
4 \\
3\end{array}$ & $\begin{array}{l}\text { INSTRUCTION } \\
\text { INSTRUCTIONS } \\
\text { INST RUMENT }\end{array}$ & $\begin{array}{l}70 \\
20 \\
10\end{array}$ & $\begin{array}{l}\text { ITS } \\
\text { ITSELF } \\
\text { IT'D }\end{array}$ \\
\hline $\begin{array}{l}2 \\
8 \\
3\end{array}$ & $\begin{array}{l}\text { INCL INED } \\
\text { INCLUDE } \\
\text { INCLUDED }\end{array}$ & $\begin{array}{l}4 \\
2 \\
2\end{array}$ & $\begin{array}{l}\text { INSURANCE } \\
\text { INSURRECTION } \\
\text { INTEGRAL }\end{array}$ & $\begin{array}{r}29 \\
1371 \\
187\end{array}$ & $\begin{array}{l}I T ` L L \\
I T \cdot S \\
I ` D\end{array}$ \\
\hline $\begin{array}{r}4 \\
13 \\
14\end{array}$ & $\begin{array}{l}\text { INCLUDES } \\
\text { INCLUDING } \\
\text { INCOME }\end{array}$ & $\begin{array}{l}8 \\
2 \\
4\end{array}$ & $\begin{array}{l}\text { INTELLECTUAL } \\
\text { INTELLECTUALLY } \\
\text { INTELLIGENCE }\end{array}$ & $\begin{array}{l}137 \\
619 \\
422\end{array}$ & $\begin{array}{l}I \cdot L L \\
I: M \\
I \cdot V E\end{array}$ \\
\hline $\begin{array}{l}4 \\
2 \\
2\end{array}$ & $\begin{array}{l}\text { INCOMP REHENSIBLE } \\
\text { INCREASE } \\
\text { INCREASED }\end{array}$ & $\begin{array}{l}6 \\
8 \\
3\end{array}$ & $\begin{array}{l}\text { INTELLIGENT } \\
\text { INTENDED } \\
\text { INTENSE }\end{array}$ & $\begin{array}{l}3 \\
2 \\
6\end{array}$ & $\begin{array}{l}\text { J } \\
\text { JAMBOREE } \\
\text { JANUARY }\end{array}$ \\
\hline $\begin{array}{r}2 \\
15 \\
7\end{array}$ & $\begin{array}{l}\text { INCREASING } \\
\text { I NCREDIBLE } \\
\text { I NCREDIBLY }\end{array}$ & $\begin{array}{l}2 \\
3 \\
3\end{array}$ & $\begin{array}{l}\text { INTENSITY } \\
\text { INTENSIVE } \\
\text { INTENTION }\end{array}$ & $\begin{array}{l}2 \\
2 \\
5\end{array}$ & $\begin{array}{l}\text { JARGON } \\
\text { JAW } \\
\text { JESUS }\end{array}$ \\
\hline $\begin{array}{r}58 \\
3 \\
3\end{array}$ & $\begin{array}{l}\text { INDEED } \\
\text { INDEPENDENT } \\
\text { INDEPENDENT LY }\end{array}$ & $\begin{array}{r}2 \\
36 \\
70\end{array}$ & $\begin{array}{l}\text { INTERCHANGE } \\
\text { INTEREST } \\
\text { INTERESTED }\end{array}$ & $\begin{array}{r}119 \\
15 \\
2\end{array}$ & $\begin{array}{l}\text { JOB } \\
\text { JOBS } \\
\text { JOB'S }\end{array}$ \\
\hline $\begin{array}{r}2 \\
16 \\
3\end{array}$ & $\begin{array}{l}\text { INDICATION } \\
\text { INDIVIDUAL } \\
\text { INDIVIDUALS }\end{array}$ & $\begin{array}{r}96 \\
3 \\
11\end{array}$ & $\begin{array}{l}\text { INTERESTING } \\
\text { INTERESTINGLY } \\
\text { INTERESTS }\end{array}$ & $\begin{array}{l}9 \\
4 \\
7\end{array}$ & $\begin{array}{l}\text { JOIN } \\
\text { JOINED } \\
\text { JOINT }\end{array}$ \\
\hline 3 & INDUSTRIAL & 6 & INTERNAL & 6 & JOKE \\
\hline $\begin{array}{r}3 \\
10\end{array}$ & $\begin{array}{l}\text { INDUSTR IES } \\
\text { I NDUSTRY }\end{array}$ & $\begin{array}{r}14 \\
2\end{array}$ & $\begin{array}{l}\text { INTERNATIONAL } \\
\text { INTERPRETATION }\end{array}$ & $\begin{array}{r}2 \\
32\end{array}$ & $\begin{array}{l}\text { JOKES } \\
\text { JOLLY }\end{array}$ \\
\hline $\begin{array}{l}2 \\
3 \\
2\end{array}$ & $\begin{array}{l}\text { INEFF ICIENT } \\
\text { INEVITABLY } \\
\text { INEXCUSABLE }\end{array}$ & $\begin{array}{l}3 \\
2 \\
4\end{array}$ & $\begin{array}{l}\text { INTERPRET ING } \\
\text { INTERRUPT } \\
\text { INTERVAL }\end{array}$ & $\begin{array}{l}6 \\
2 \\
2\end{array}$ & $\begin{array}{l}\text { JUDGE } \\
\text { JUDGES } \\
\text { JUDGING }\end{array}$ \\
\hline $\begin{array}{l}2 \\
3 \\
2\end{array}$ & $\begin{array}{l}\text { INEXPERIENCE } \\
\text { INFANTS } \\
\text { INFERIOR }\end{array}$ & $\begin{array}{r}37 \\
9 \\
4\end{array}$ & $\begin{array}{l}\text { INTERVI EW } \\
\text { INTERVI EWED } \\
\text { INTERVI EWING }\end{array}$ & $\begin{array}{r}18 \\
2 \\
2\end{array}$ & $\begin{array}{l}\text { JULY } \\
\text { JUMBLE } \\
\text { JUMPS }\end{array}$ \\
\hline $\begin{array}{l}4 \\
4 \\
4\end{array}$ & $\begin{array}{l}\text { INF INIT IES } \\
\text { INFINITY } \\
\text { INFLECTED }\end{array}$ & $\begin{array}{r}217 \\
4 \\
3\end{array}$ & $\begin{array}{l}\text { INTO } \\
\text { INTOLERABLE } \\
\text { INTR IGUED }\end{array}$ & $\begin{array}{r}3 \\
14 \\
8\end{array}$ & $\begin{array}{l}\text { JUNCTION } \\
\text { JUNE } \\
\text { JUNIOR }\end{array}$ \\
\hline $\begin{array}{l}2 \\
2 \\
3\end{array}$ & $\begin{array}{l}\text { INFLUENCE } \\
\text { INFLUENCED } \\
\text { INFORM }\end{array}$ & $\begin{array}{r}4 \\
11 \\
3\end{array}$ & $\begin{array}{l}\text { INTRODUCE } \\
\text { INTRODUCED } \\
\text { INTRODUCING }\end{array}$ & $\begin{array}{r}670 \\
2 \\
2\end{array}$ & $\begin{array}{l}\text { JUST } \\
\text { JUST IFIED } \\
\text { JUSTIFY }\end{array}$ \\
\hline 2 & INFORMAL & 2 & INVADED & 7 & $\mathrm{~K}$ \\
\hline $\begin{array}{l}8 \\
2\end{array}$ & $\begin{array}{l}\text { INFORMATION } \\
\text { I NFORMED }\end{array}$ & $\begin{array}{l}2 \\
3\end{array}$ & $\begin{array}{l}\text { INVALIDED } \\
\text { INVENTED }\end{array}$ & $\begin{array}{r}4 \\
13\end{array}$ & $\begin{array}{l}\text { KANGAROO } \\
\text { KEEN }\end{array}$ \\
\hline $\begin{array}{l}2 \\
2 \\
3\end{array}$ & $\begin{array}{l}\text { INFUR IATED } \\
\text { INHERITANCE } \\
\text { I NHERITED }\end{array}$ & $\begin{array}{l}4 \\
2 \\
3\end{array}$ & $\begin{array}{l}\text { INVEST } \\
\text { INVESTED } \\
\text { INVESTIGATION }\end{array}$ & $\begin{array}{r}56 \\
6 \\
9\end{array}$ & $\begin{array}{l}\text { KEEP } \\
\text { KEEP ING } \\
\text { KEEPS }\end{array}$ \\
\hline $\begin{array}{l}7 \\
4 \\
3\end{array}$ & $\begin{array}{l}\text { INITIAL } \\
\text { INITIALLY } \\
\text { INNOCENT }\end{array}$ & $\begin{array}{r}5 \\
3 \\
10\end{array}$ & $\begin{array}{l}\text { INVESTMENT } \\
\text { INVITATION } \\
\text { INVITED }\end{array}$ & $\begin{array}{r}33 \\
7 \\
19\end{array}$ & $\begin{array}{l}\text { KEPT } \\
\text { KETTLE } \\
\text { KEY }\end{array}$ \\
\hline 2 & INNOCUOUS & 2 & INVOLVE & 5 & KEYS \\
\hline $\begin{array}{r}2 \\
16\end{array}$ & $\begin{array}{l}\text { INSERTING } \\
\text { INSIDE }\end{array}$ & $\begin{array}{r}16 \\
2\end{array}$ & $\begin{array}{l}\text { INVOLVED } \\
\text { INVOLVES }\end{array}$ & $\begin{array}{l}3 \\
2\end{array}$ & $\begin{array}{l}\text { KICK } \\
\text { KICKED }\end{array}$ \\
\hline
\end{tabular}


Appendix A (Continued)

\begin{tabular}{|c|c|c|c|c|c|}
\hline $\begin{array}{r}15 \\
2\end{array}$ & $\begin{array}{l}\text { KID } \\
\text { KIDDY }\end{array}$ & $\begin{array}{l}6 \\
4\end{array}$ & $\begin{array}{l}\text { LAUGH } \\
\text { LAUGHED }\end{array}$ & $\begin{array}{r}12 \\
2\end{array}$ & $\begin{array}{l}\text { LIGHT } \\
\text { LIGHTER }\end{array}$ \\
\hline $\begin{array}{r}21 \\
4 \\
9\end{array}$ & $\begin{array}{l}\text { KIDS } \\
\text { KILL } \\
\text { KILLED }\end{array}$ & $\begin{array}{l}6 \\
2 \\
4\end{array}$ & $\begin{array}{l}\text { LAUGHING } \\
\text { LAVATORIES } \\
\text { LAVATORY }\end{array}$ & $\begin{array}{r}2 \\
2 \\
600\end{array}$ & $\begin{array}{l}\text { LIGHTING } \\
\text { LIGHTS } \\
\text { LIKE }\end{array}$ \\
\hline $\begin{array}{r}5 \\
150 \\
4\end{array}$ & $\begin{array}{l}\text { KILLING } \\
\text { KIND } \\
\text { KINDLY }\end{array}$ & $\begin{array}{r}23 \\
3 \\
2\end{array}$ & $\begin{array}{l}\text { LAW } \\
\text { LAWYERS } \\
\text { LAWYER'S }\end{array}$ & $\begin{array}{l}14 \\
13 \\
11\end{array}$ & $\begin{array}{l}\text { LIKED } \\
\text { LIKELY } \\
\text { LIKES }\end{array}$ \\
\hline 2 & KINDNESS & 4 & LAYMAN & 5 & LIKING \\
\hline 14 & KINDS & 6 & LAYOUT & 4 & LIMIT \\
\hline 4 & KING & 3 & LAZY & 5 & LIMITED \\
\hline 2 & KINGDOM & 3 & LEAD & 36 & LINE \\
\hline 3 & KINGF ISHER & 11 & $\begin{array}{l}\text { LEAD ING } \\
\text { LFARN }\end{array}$ & 2 & LINEAR \\
\hline 2 & KINGS & 18 & LEARN & 4 & LINED \\
\hline $\begin{array}{r}3 \\
10\end{array}$ & $\begin{array}{l}\text { KING`S } \\
\text { KIT }\end{array}$ & $\begin{array}{l}4 \\
9\end{array}$ & $\begin{array}{l}\text { LEARNING } \\
\text { LEARNT }\end{array}$ & $\begin{array}{r}2 \\
20\end{array}$ & $\begin{array}{l}\text { LINEN } \\
\text { LINES }\end{array}$ \\
\hline 9 & KITCHEN & 49 & LEAST & 5 & LINGUAPHONE \\
\hline 3 & KITS & 54 & LEAVE & 5 & LINGUIST \\
\hline 4 & KNEES & 4 & LEAVES & $\begin{array}{l}6 \\
3\end{array}$ & $\begin{array}{l}\text { L INGUISTIC } \\
\text { LINGUIST ICALLY }\end{array}$ \\
\hline 86 & KNEW & 9 & LEAV I NG & 3 & LINGUIST ICALLY \\
\hline $\begin{array}{l}4 \\
3\end{array}$ & KNIGHT & 41 & LECTURE & 33 & LINGUISTICS \\
\hline $\begin{array}{r}3 \\
11\end{array}$ & $\begin{array}{l}\text { KNITTED } \\
\text { KNITTING }\end{array}$ & $\begin{array}{r}37 \\
7\end{array}$ & $\begin{array}{l}\text { LECTURER } \\
\text { LECTURERS }\end{array}$ & $\begin{array}{l}2 \\
2\end{array}$ & $\begin{array}{l}\text { LINGUISTS } \\
\text { LINK }\end{array}$ \\
\hline 2 & KNOCK & 37 & LECTURES & 3 & L INKS \\
\hline 6 & KNOCKED & 12 & LECTURING & 4 & LIQUID \\
\hline 4 & KNOCKING & 16 & LED & 27 & LIS $\mathrm{T}$ \\
\hline 1621 & KNOW & 82 & LEFT & 3 & LISTED \\
\hline 15 & KNOW ING & 3 & LEFTHAND & 13 & LISTEN \\
\hline 17 & KNOWLEDGE & 2 & LEGACY & 4 & LISTENED \\
\hline 37 & KNOWN & 2 & LEGAL & 2 & LISTENERS \\
\hline $\begin{array}{r}30 \\
3\end{array}$ & KNOWS & 3 & $\begin{array}{l}\text { LEGALLY } \\
\text { LEGS }\end{array}$ & 10 & $\begin{array}{l}\text { LISTENING } \\
\text { LIT }\end{array}$ \\
\hline $\begin{array}{r}3 \\
10\end{array}$ & $\begin{array}{l}\text { L } \\
\text { LABEL }\end{array}$ & $\begin{array}{l}5 \\
5\end{array}$ & $\begin{array}{l}\text { LEGS } \\
\text { LEMON }\end{array}$ & $\begin{array}{r}5 \\
19\end{array}$ & $\begin{array}{l}\text { LIT } \\
\text { LITERALLY }\end{array}$ \\
\hline 5 & LABOUR & 7 & LEND & 19 & LITERARY \\
\hline 4 & LACK & 5 & LENGTH & 70 & LITERATURE \\
\hline 3 & LACK I NG & 3 & LENS & 193 & LITTLE \\
\hline 3 & LAD & 2 & LENSES & 43 & LIVE \\
\hline 2 & LADDER & 2 & LENT & 20 & LIVED \\
\hline 5 & LADIES & 56 & LESS & 2 & LIVELY \\
\hline $\begin{array}{l}3 \\
2\end{array}$ & $\begin{array}{l}\text { LADIES } \\
\text { LADS }\end{array}$ & $\begin{array}{r}3 \\
64\end{array}$ & $\begin{array}{l}\text { LESSONS } \\
\text { LET }\end{array}$ & $\begin{array}{l}16 \\
53\end{array}$ & $\begin{array}{l}\text { LIVES } \\
\text { LIVING }\end{array}$ \\
\hline 9 & LADY & 2 & LETHAL & 4 & LOAD \\
\hline $\begin{array}{l}6 \\
3\end{array}$ & $\begin{array}{l}\text { LAGER } \\
\text { LAGERS }\end{array}$ & $\begin{array}{r}3 \\
46\end{array}$ & $\begin{array}{l}\text { LETS } \\
\text { LETTER }\end{array}$ & $\begin{array}{r}5 \\
16\end{array}$ & $\begin{array}{l}\text { LOAN } \\
\text { LOCAL }\end{array}$ \\
\hline 3 & LAID & 26 & LETTERS & 4 & LOCK \\
\hline 6 & LAND & 3 & LETT ING & 2 & LOCKER \\
\hline 3 & LANDED & 39 & LET'S & 2 & LOG \\
\hline $\begin{array}{r}6 \\
68\end{array}$ & $\begin{array}{l}\text { LANDSCAPE } \\
\text { LANGUAGE }\end{array}$ & $\begin{array}{r}20 \\
2\end{array}$ & $\begin{array}{l}\text { LEVEL } \\
\text { LEXICAL }\end{array}$ & $\begin{array}{l}2 \\
4\end{array}$ & $\begin{array}{l}\text { LOGGER } \\
\text { LOGICAL }\end{array}$ \\
\hline 17 & LANGUAGES & 3 & LEX I COGRAPHY & 6 & LONELY \\
\hline 28 & LARGE & 2 & LEXICOLOGY & 135 & LONG \\
\hline $\begin{array}{l}7 \\
2\end{array}$ & $\begin{array}{l}\text { LARGELY } \\
\text { LARGEST }\end{array}$ & $\begin{array}{l}4 \\
2\end{array}$ & $\begin{array}{l}\text { LIBRAR IAN } \\
\text { LIBRAR IES }\end{array}$ & $\begin{array}{r}20 \\
3\end{array}$ & $\begin{array}{l}\text { LONGER } \\
\text { LONGING }\end{array}$ \\
\hline 2 & LARK & 55 & LIBRARY & 2 & LONGSCALE \\
\hline 2 & LARKING & 2 & LICENCES & 2 & LOO \\
\hline 184 & LAST & 2 & LIES & 156 & LOOK \\
\hline 36 & LATE & 43 & LIFE & 45 & LOOKED \\
\hline 7 & LATELY & 2 & LIFETIME & 51 & $\begin{array}{l}\text { LOOKING } \\
\text { LOOKS }\end{array}$ \\
\hline 33 & $\begin{array}{l}\text { LATER } \\
\text { LATTER }\end{array}$ & 7 & $\begin{array}{l}\text { LIFT } \\
\text { LIFTED }\end{array}$ & 39 & $\begin{array}{l}\text { LOOKS } \\
\text { LOOPHOLE }\end{array}$ \\
\hline 4 & LATTER & 2 & LIFTED & 2 & \\
\hline
\end{tabular}


Appendix A (Continued)

\begin{tabular}{|c|c|c|c|c|c|}
\hline 12 & LOOPHOLES & 138 & MAN & 2 & MEAT \\
\hline $\begin{array}{l}2 \\
3 \\
2\end{array}$ & $\begin{array}{l}\text { LOOS } \\
\text { LOOSE } \\
\text { LORDS }\end{array}$ & $\begin{array}{r}5 \\
7 \\
16\end{array}$ & $\begin{array}{l}\text { MANAGE } \\
\text { MANAGED } \\
\text { MANAGEMENT }\end{array}$ & $\begin{array}{r}2 \\
12 \\
5\end{array}$ & $\begin{array}{l}\text { MECHANISM } \\
\text { MEDIA } \\
\text { MEDICAL }\end{array}$ \\
\hline 2 & LORRIES & 5 & MANAGER & 21 & MEDICINE \\
\hline 17 & LOSE & 4 & MANAGER' $S$ & 20 & MEDI EVAL \\
\hline 3 & LOSS & 2 & MANIFEST & 2 & MEDI EVALIST \\
\hline 24 & LOST & 5 & MANNER & 2 & MEDI OCRE \\
\hline 218 & LOT & 2 & MANSE & 4 & MEDIUM \\
\hline 36 & LOTS & 2 & MANUF ACTUR ING & 30 & MEET \\
\hline 3 & LOUD & 5 & MANUSCRIPT & 42 & MEET ING \\
\hline $\begin{array}{r}3 \\
26\end{array}$ & $\begin{array}{l}\text { LOUSY } \\
\text { LOVE }\end{array}$ & $\begin{array}{r}2 \\
95\end{array}$ & $\begin{array}{l}\text { MANUSCRIPTS } \\
\text { MANY }\end{array}$ & $\begin{array}{l}2 \\
2\end{array}$ & $\begin{array}{l}\text { MEETINGS } \\
\text { MEETS }\end{array}$ \\
\hline 5 & LOVED & 5 & MAN'S & 2 & MELODIE \\
\hline 70 & LOVELY & 20 & MAP & 2 & MELT \\
\hline 7 & LOVERS & 6 & MAPS & 16 & MEMBER \\
\hline $\begin{array}{l}4 \\
7 \\
3\end{array}$ & $\begin{array}{l}\text { LOVES } \\
\text { LOW } \\
\text { LOWER }\end{array}$ & $\begin{array}{l}6 \\
4 \\
2\end{array}$ & $\begin{array}{l}\text { MARCH } \\
\text { MARGINAL } \\
\text { MARG INALLY }\end{array}$ & $\begin{array}{r}14 \\
6 \\
29\end{array}$ & $\begin{array}{l}\text { MEMBERS } \\
\text { MEMORY } \\
\text { MEN }\end{array}$ \\
\hline 3 & LPO & 17 & MARK & 4 & MENTAL \\
\hline $\begin{array}{l}2 \\
4\end{array}$ & $\begin{array}{l}\text { LSE } \\
\text { LUCK }\end{array}$ & $\begin{array}{l}6 \\
9\end{array}$ & $\begin{array}{l}\text { MARKED } \\
\text { MARKET }\end{array}$ & $\begin{array}{l}2 \\
2\end{array}$ & $\begin{array}{l}\text { MENTALITY } \\
\text { MENTHOL }\end{array}$ \\
\hline 2 & LUCKILY & 3 & MARKING & 18 & MENTION \\
\hline $\begin{array}{l}6 \\
3\end{array}$ & $\begin{array}{l}\text { LUCKY } \\
\text { LUMBERED }\end{array}$ & $\begin{array}{r}13 \\
3\end{array}$ & $\begin{array}{l}\text { MARKS } \\
\text { MARRIAGE }\end{array}$ & $\begin{array}{r}25 \\
2\end{array}$ & $\begin{array}{l}\text { MENT IONED } \\
\text { MENT IONING }\end{array}$ \\
\hline 28 & I.UNCH & 38 & MARRIED & 4 & $M E N^{\prime} S$ \\
\hline 2 & LUNCHED & 3 & MARRY & 23 & MERELY \\
\hline 5 & LUNCHTIME & 17 & MARVELLOUS & 2 & MERGE \\
\hline 10 & LUNG & 4 & MASKED & 9 & MESS \\
\hline 2 & LYING & 5 & MASS & 5 & MESSAGE \\
\hline 22 & M & 3 & MASSES & 2 & MESSAGES \\
\hline 9 & MA & 5 & MASTER & 46 & MET \\
\hline 23 & MACHINE & 6 & MATCH & 2 & METAL \\
\hline 3 & MACH INES & 2 & MATE & 5 & METHOD \\
\hline 6 & MACROBIOTICS & 25 & MATERIAL & 5 & METHODS \\
\hline 2 & MACROSCOPIC & 3 & MATERIALS & 5 & METRE \\
\hline 16 & MAD & 3 & MATHEMAT ICI AN & 2 & METRES \\
\hline 2 & MADDENING & 2 & MATHEMATICIANS & 4 & MI \\
\hline 100 & MADE & 13 & MATHEMATICS & 3 & MICE \\
\hline 2 & MADLY & 6 & MATHS & 2 & M ICROF I LMS \\
\hline 3 & MADNESS & 39 & MATTER & 2 & MICROPHONES \\
\hline $\begin{array}{l}7 \\
3\end{array}$ & $\begin{array}{l}\text { MAGAZINE } \\
\text { MAGAZ INES }\end{array}$ & $\begin{array}{l}8 \\
3\end{array}$ & $\begin{array}{l}\text { MATTERS } \\
\text { MATTRESS }\end{array}$ & $\begin{array}{r}3 \\
44\end{array}$ & $\begin{array}{l}\text { MID } \\
\text { MIDDLE }\end{array}$ \\
\hline 3 & MAGIC & 3 & MATURED & 2 & MIDDLEAGED \\
\hline $\begin{array}{r}3 \\
32\end{array}$ & $\begin{array}{l}\text { MAGNIFICENT } \\
\text { MAIN }\end{array}$ & $\begin{array}{r}3 \\
157\end{array}$ & $\begin{array}{l}\text { MAXIMUM } \\
\text { MAY }\end{array}$ & $\begin{array}{r}2 \\
143\end{array}$ & $\begin{array}{l}\text { MIDGET } \\
\text { MIGHT }\end{array}$ \\
\hline 5 & MAINLY & 24 & MAYBE & 3 & MIGHTN`T \\
\hline 7 & MAINTAIN & 528 & ME & 4 & MILD \\
\hline 13 & MAJOR & 5 & MEAR & 9 & MILES \\
\hline $\begin{array}{r}6 \\
147 \\
4\end{array}$ & $\begin{array}{l}\text { MAJORITY } \\
\text { MAKE } \\
\text { MAKEN }\end{array}$ & $\begin{array}{r}8 \\
3 \\
842\end{array}$ & $\begin{array}{l}\text { MEAL } \\
\text { MEALS } \\
\text { MEAN }\end{array}$ & $\begin{array}{r}2 \\
9 \\
12\end{array}$ & $\begin{array}{l}\text { MILITARISTIC } \\
\text { MILITARY } \\
\text { MILK }\end{array}$ \\
\hline 2 & MAKER & 13 & MEANING & 11 & MILLION \\
\hline $\begin{array}{l}37 \\
26\end{array}$ & $\begin{array}{l}\text { MAKES } \\
\text { MAKING }\end{array}$ & $\begin{array}{r}2 \\
53\end{array}$ & $\begin{array}{l}\text { MEANINGLESS } \\
\text { MEANS }\end{array}$ & $\begin{array}{l}2 \\
5\end{array}$ & $\begin{array}{l}\text { MILLIONA IRES } \\
\text { MILIIONS }\end{array}$ \\
\hline 3 & MALARIA & 40 & MEANT & 99 & MIND \\
\hline 2 & MALICIOUS & 2 & MEANT IME & 3 & MINDS \\
\hline 2 & MALIGNANT & 4 & MEANWHILE & 30 & MINE \\
\hline $\begin{array}{l}2 \\
2\end{array}$ & $\begin{array}{l}\text { MALT } \\
\text { MAM }\end{array}$ & $\begin{array}{l}5 \\
3\end{array}$ & $\begin{array}{l}\text { MEASURE } \\
\text { MEASUREMENTS }\end{array}$ & $\begin{array}{l}3 \\
2\end{array}$ & $\begin{array}{l}\text { MINERS } \\
\text { MINESTRA }\end{array}$ \\
\hline
\end{tabular}


Appendix A (Continued)

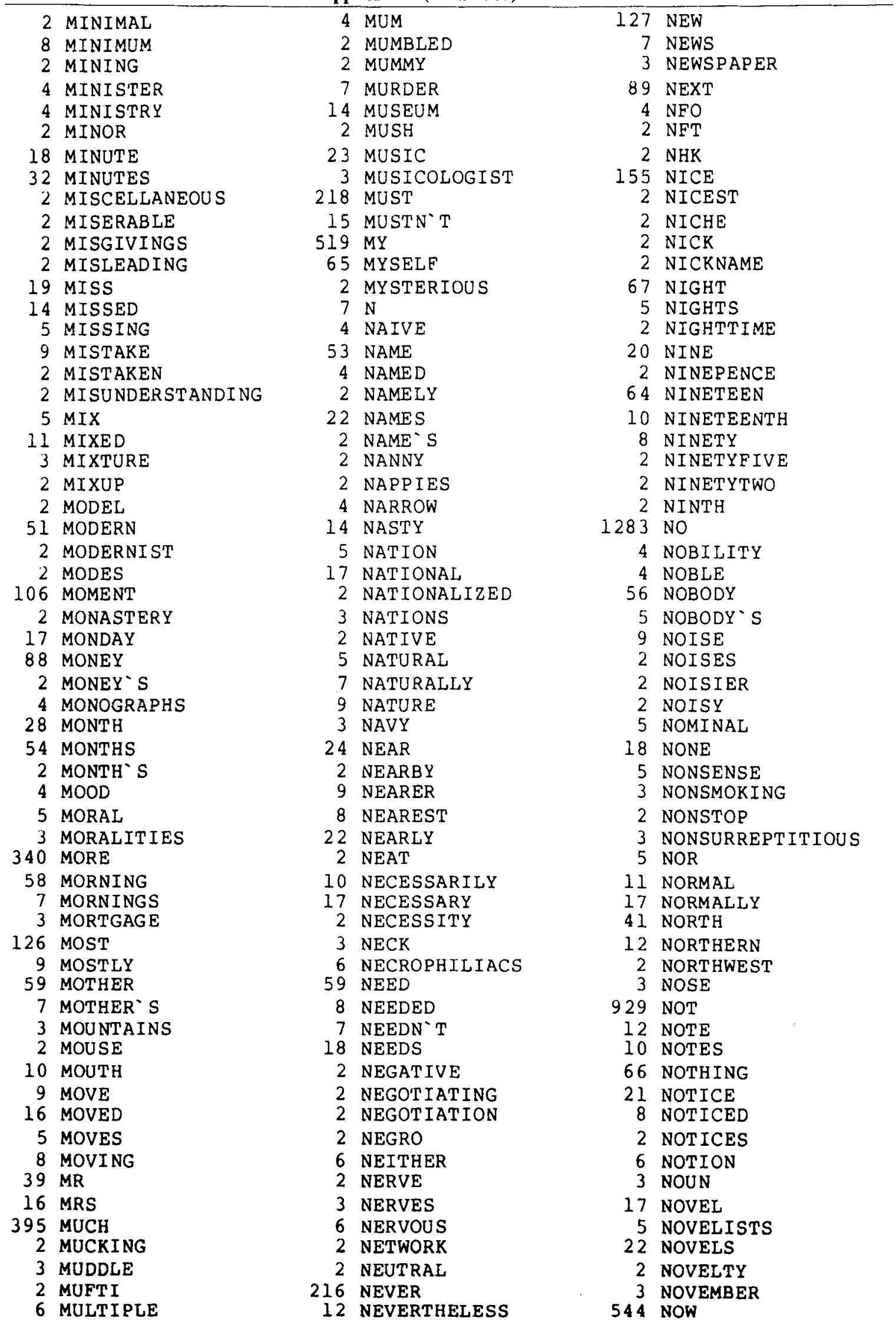


Appendix A (Continued)

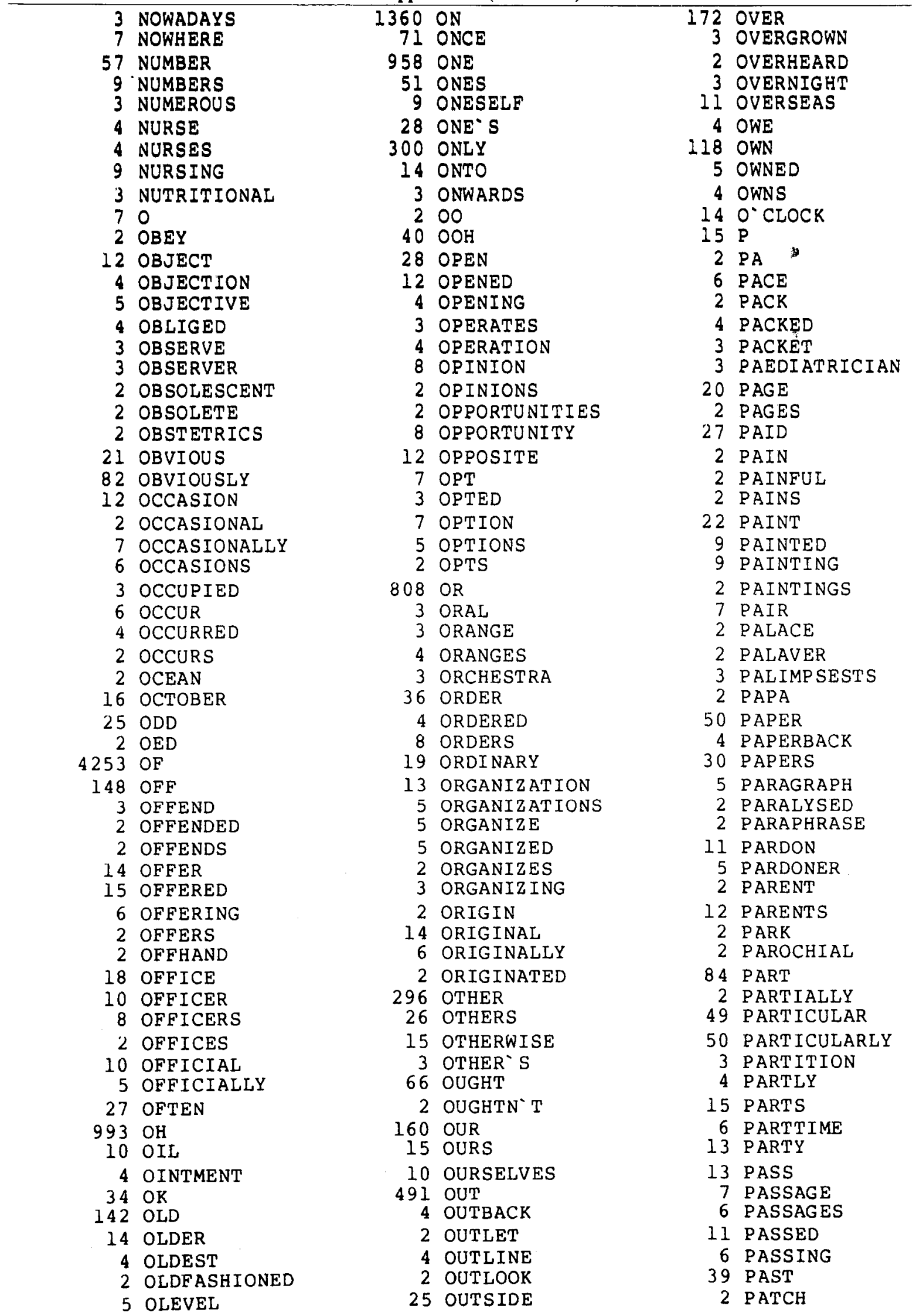


Appendix A (Continued)

\begin{tabular}{|c|c|c|c|c|c|}
\hline 5 & PATHETIC & 3 & PHONETIC & 36 & POETRY \\
\hline 2 & PATHOLOGICAL & 2 & PHOTOCOPYING & 6 & POETS \\
\hline 21 & $\begin{array}{l}\text { PATIENT } \\
\text { PATIENTS }\end{array}$ & $\begin{array}{l}3 \\
2\end{array}$ & $\begin{array}{l}\text { PHOTOGRAPH } \\
\text { PHOTOGRAPHIC }\end{array}$ & $14 \frac{1}{5}$ & $\begin{array}{l}\text { POINT } \\
\text { POINTED }\end{array}$ \\
\hline $\begin{array}{r}2 \\
8 \\
34\end{array}$ & $\begin{array}{l}\text { PATRISTICS } \\
\text { PATTERN } \\
\text { PAY }\end{array}$ & $\begin{array}{l}2 \\
4 \\
2\end{array}$ & $\begin{array}{l}\text { PHO'OOGRAPHS } \\
\text { PHRASE } \\
\text { PHRASES }\end{array}$ & $\begin{array}{r}3 \\
12 \\
3\end{array}$ & $\begin{array}{l}\text { POINTLESS } \\
\text { POINTS } \\
\text { POISONOUS }\end{array}$ \\
\hline $\begin{array}{r}10 \\
4 \\
7\end{array}$ & $\begin{array}{l}\text { PAYING } \\
\text { PAYS } \\
\text { PEACE }\end{array}$ & $\begin{array}{l}3 \\
4 \\
2\end{array}$ & $\begin{array}{l}\text { PHYSICAL } \\
\text { PHYSICIAN } \\
\text { PHYSICS }\end{array}$ & $\begin{array}{r}3 \\
9 \\
18\end{array}$ & $\begin{array}{l}\text { POKING } \\
\text { POLICE } \\
\text { POLICY }\end{array}$ \\
\hline $\begin{array}{l}7 \\
8\end{array}$ & $\begin{array}{l}\text { PEACET IME } \\
\text { PEAK }\end{array}$ & $\begin{array}{r}3 \\
18\end{array}$ & $\begin{array}{l}\text { PHYSIOLOGICAL } \\
\text { PICK }\end{array}$ & $\begin{array}{l}2 \\
9\end{array}$ & $\begin{array}{l}\text { POLISH } \\
\text { POLITE }\end{array}$ \\
\hline $\begin{array}{l}8 \\
2\end{array}$ & $\begin{array}{l}\text { PEAK } \\
\text { PEARS }\end{array}$ & $\begin{array}{r}18 \\
8\end{array}$ & $\begin{array}{l}\text { PICK } \\
\text { PICKED }\end{array}$ & 11 & POLITICAE \\
\hline 10 & PECULIAR & 5 & PICKING & 2 & POLITICALLY \\
\hline $\begin{array}{l}2 \\
2\end{array}$ & $\begin{array}{l}\text { PECULIARS } \\
\text { PEDESTRIAN }\end{array}$ & $\begin{array}{r}2 \\
24\end{array}$ & $\begin{array}{l}\text { PICKLED } \\
\text { PICTURE }\end{array}$ & $\begin{array}{l}7 \\
3\end{array}$ & $\begin{array}{l}\text { POLITICS } \\
\text { POLYTECHNIC }\end{array}$ \\
\hline 2 & PEN & 15 & PICTURES & 2 & POOL \\
\hline 5 & $\begin{array}{l}\text { PENCE } \\
\text { PENINSULA }\end{array}$ & $\begin{array}{r}12 \\
5\end{array}$ & $\begin{array}{l}\text { PIECE } \\
\text { PIECES }\end{array}$ & $\begin{array}{r}19 \\
8\end{array}$ & $\begin{array}{l}\text { POOR } \\
\text { POP }\end{array}$ \\
\hline $\begin{array}{r}2 \\
5 \\
533\end{array}$ & $\begin{array}{l}\text { PENNI LESS } \\
\text { PENNY } \\
\text { PEOPLE }\end{array}$ & $\begin{array}{l}2 \\
3 \\
2\end{array}$ & $\begin{array}{l}\text { PIGEONHOLE } \\
\text { PIGSTY } \\
\text { PILED }\end{array}$ & $\begin{array}{l}5 \\
2 \\
3\end{array}$ & $\begin{array}{l}\text { POPE } \\
\text { POPERY } \\
\text { POPPET }\end{array}$ \\
\hline 12 & PEOPLE'S & 3 & PINK & 2 & POPULACE \\
\hline 26 & PER & 3 & PINT & 9 & POPULAR \\
\hline 2 & PERCENTAGE & 2 & PINTS & 3 & POPULATION \\
\hline 2 & PERCUSSING & 21 & PIP & 9 & PORT \\
\hline $\begin{array}{r}4 \\
30\end{array}$ & $\begin{array}{l}\text { PERFECT } \\
\text { PERFECTLY }\end{array}$ & $\begin{array}{l}5 \\
2\end{array}$ & $\begin{array}{l}\text { PIPE } \\
\text { PIPELINE }\end{array}$ & $\begin{array}{l}4 \\
2\end{array}$ & $\begin{array}{l}\text { PORTER } \\
\text { PORTERS }\end{array}$ \\
\hline 40 & PERFORMANCE & 2 & PIT & 7 & PORTRAIT \\
\hline $\begin{array}{r}2 \\
122\end{array}$ & $\begin{array}{l}\text { PERFORMANCES } \\
\text { PERHAQS }\end{array}$ & $\begin{array}{r}14 \\
123\end{array}$ & $\begin{array}{l}\text { PITY } \\
\text { PLACE }\end{array}$ & $\begin{array}{r}13 \\
6\end{array}$ & $\begin{array}{l}\text { PORTRAITS } \\
\text { POSH }\end{array}$ \\
\hline 2 & PERILOUS & 28 & PLACES & 25 & POSITION \\
\hline 31 & PERIOD & 4 & PLAIN & 3 & POSITIVE \\
\hline 2 & PERIODS & 5 & PLAN & 2 & POSSIBILITIES \\
\hline 11 & PERMANENT & 4 & PLANE & 14 & POSSIBILITY \\
\hline 4 & PERMANENTLY & 2 & PLANNED & 38 & POSSIBLE \\
\hline 4 & PERMISSION & 7 & PLANNING & 28 & POSSIBLY \\
\hline 5 & PERMIT & 4 & PLANS & 21 & POST \\
\hline 64 & PERSON & 7 & PLASTIC & 2 & POSTCARD \\
\hline 14 & PERSONAL & 4 & PLASTICS & 2 & POSTED \\
\hline 17 & PERSONALLY & 2 & PLATE & 2 & POSTERITY \\
\hline 3 & PERSONS & 3 & PLATES & 10 & POSTGRADUATE \\
\hline 5 & PERSPECTIVE & 3 & PLATFORM & 4 & POSTGRADUATES \\
\hline 4 & PERSUADE & 64 & PLAY & 2 & POSTING \\
\hline 4 & PERSUADED & 6 & PLAYED & 4 & POSTMEDI EVAL \\
\hline 2 & PERSUASIVE & 10 & PLAY ING & 5 & POSTS \\
\hline 3 & PETROL & 20 & PLAYS & 3 & POTENTIAL \\
\hline 3 & PHASE & 11 & PLEASANT & 2 & POTS \\
\hline 19 & PHD & 25 & PLEASE & 3 & POTTERY \\
\hline 2 & PHENOMENAL & 5 & PLEASED & 8 & POUND \\
\hline 2 & PHEW & 11 & PLEASURE & 59 & POUNDS \\
\hline 3 & PHILISTINE & 13 & PLENTY & 2 & POUR \\
\hline 2 & PHILOLOGIST & 2 & PLUG & 4 & POURED \\
\hline 5 & PH ILOLOGY & 3 & PLURAL & 3 & POUR ING \\
\hline 2 & PHILOSOPHICAL & 14 & PLUS & 6 & POWER \\
\hline 4 & PHILOSOPHY & 2 & POCKET & 3 & POWERFUL \\
\hline $2 I$ & PHONE & 7 & POEM & 2 & $\mathrm{PP}$ \\
\hline 2 & PHONEBOX & 3 & POEMS & 10 & PRACTICAL \\
\hline 5 & PHONED & 2 & POET & 16 & PRACT I CALLY \\
\hline 2 & PHONEMIC & 2 & POETIC & 7 & PRACTICE \\
\hline
\end{tabular}


Appendix A (Continued)

\begin{tabular}{|c|c|c|c|c|c|}
\hline $\begin{array}{l}2 \\
2 \\
2\end{array}$ & $\begin{array}{l}\text { PRACTISE } \\
\text { PRACTITIONER } \\
\text { PRAISE }\end{array}$ & $\begin{array}{l}2 \\
5 \\
7\end{array}$ & $\begin{array}{l}\text { PRINTER } \\
\text { PRINTING } \\
\text { PRIORITY }\end{array}$ & $\begin{array}{r}15 \\
6 \\
2\end{array}$ & $\begin{array}{l}\text { PROVIDE } \\
\text { PROVIDED } \\
\text { PROVIDENCE }\end{array}$ \\
\hline $\begin{array}{l}2 \\
2 \\
2\end{array}$ & $\begin{array}{l}\text { PRAISES } \\
\text { PRECEDENTS } \\
\text { PRECIS }\end{array}$ & $\begin{array}{r}3 \\
3 \\
33\end{array}$ & $\begin{array}{l}\text { PRISON } \\
\text { PRIVACY } \\
\text { PRIVATE }\end{array}$ & $\begin{array}{l}2 \\
4 \\
2\end{array}$ & $\begin{array}{l}\text { PROVIDING } \\
\text { PROVISION } \\
\text { PROVOST }\end{array}$ \\
\hline $\begin{array}{l}4 \\
7 \\
2\end{array}$ & $\begin{array}{l}\text { PRECISE } \\
\text { PRECISELY } \\
\text { PRECISION }\end{array}$ & $\begin{array}{r}5 \\
170 \\
4\end{array}$ & $\begin{array}{l}\text { PRO } \\
\text { PROBABLY } \\
\text { PROBATION }\end{array}$ & $\begin{array}{l}2 \\
3 \\
3\end{array}$ & $\begin{array}{l}\text { PS } \\
\text { PSALTER } \\
\text { PSYCHOLOGISTS }\end{array}$ \\
\hline $\begin{array}{l}3 \\
2 \\
9\end{array}$ & $\begin{array}{l}\text { PREDICTED } \\
\text { PREDICT ING } \\
\text { PREFER }\end{array}$ & $\begin{array}{r}42 \\
19 \\
3\end{array}$ & $\begin{array}{l}\text { PROBLEM } \\
\text { PROBLEMS } \\
\text { PROCEDURE }\end{array}$ & $\begin{array}{r}2 \\
9 \\
22\end{array}$ & $\begin{array}{l}\text { PSYCHOLOGY } \\
\text { PUB } \\
\text { PUBLIC }\end{array}$ \\
\hline $\begin{array}{l}2 \\
2 \\
2\end{array}$ & $\begin{array}{l}\text { PREFERENCE } \\
\text { PREGNANT } \\
\text { PREH ISTORIC }\end{array}$ & $\begin{array}{l}2 \\
2 \\
5\end{array}$ & $\begin{array}{l}\text { PROCEEDED } \\
\text { PROCEEDINGS } \\
\text { PROCEEDS }\end{array}$ & $\begin{array}{l}5 \\
4 \\
3\end{array}$ & $\begin{array}{l}\text { PUBLICATIONS } \\
\text { PUBLICITY } \\
\text { PUBLISH }\end{array}$ \\
\hline $\begin{array}{l}2 \\
5 \\
2\end{array}$ & $\begin{array}{l}\text { PREJUDICED } \\
\text { PREMISES } \\
\text { PREPARATION }\end{array}$ & $\begin{array}{r}9 \\
2 \\
18\end{array}$ & $\begin{array}{l}\text { PROCESS } \\
\text { PROCESSES } \\
\text { PRODUCE }\end{array}$ & $\begin{array}{l}5 \\
5 \\
2\end{array}$ & $\begin{array}{l}\text { PUBLISHED } \\
\text { PUBLISHER } \\
\text { PUBLISHERS }\end{array}$ \\
\hline $\begin{array}{r}4 \\
22 \\
11\end{array}$ & $\begin{array}{l}\text { PREPARE } \\
\text { PREPARED } \\
\text { PREPARING }\end{array}$ & $\begin{array}{r}13 \\
6 \\
4\end{array}$ & $\begin{array}{l}\text { PRODUCED } \\
\text { PRODUCING } \\
\text { PRODUCT }\end{array}$ & $\begin{array}{r}5 \\
5 \\
18\end{array}$ & $\begin{array}{l}\text { PUBLISHING } \\
\text { PUBS } \\
\text { PULL }\end{array}$ \\
\hline $\begin{array}{l}2 \\
2 \\
2\end{array}$ & $\begin{array}{l}\text { PREPOSITIONAL } \\
\text { PREPOSITIONS } \\
\text { PREREQUISITE }\end{array}$ & $\begin{array}{l}6 \\
2 \\
7\end{array}$ & $\begin{array}{l}\text { PRODUCTION } \\
\text { PRODUCTS } \\
\text { PROFESSION }\end{array}$ & $\begin{array}{l}7 \\
3 \\
2\end{array}$ & $\begin{array}{l}\text { PULLED } \\
\text { PULLING } \\
\text { PULLS }\end{array}$ \\
\hline $\begin{array}{r}2 \\
2 \\
34\end{array}$ & $\begin{array}{l}\text { PRESCRIBE } \\
\text { PRESENCE } \\
\text { PRESENT }\end{array}$ & $\begin{array}{r}5 \\
44 \\
5\end{array}$ & $\begin{array}{l}\text { PROFESSIONAL } \\
\text { PROFESSOR } \\
\text { PROFESSORS }\end{array}$ & $\begin{array}{l}2 \\
7 \\
3\end{array}$ & $\begin{array}{l}\text { PUMPED } \\
\text { PUNCTUATION } \\
\text { PURCHASE }\end{array}$ \\
\hline $\begin{array}{l}3 \\
7 \\
2\end{array}$ & $\begin{array}{l}\text { PRESENTATION } \\
\text { PRESENTDAY } \\
\text { PRESENTED }\end{array}$ & $\begin{array}{r}2 \\
19 \\
8\end{array}$ & $\begin{array}{l}\text { PROF ITABLE } \\
\text { PROGRAMME } \\
\text { PROGRAMMES }\end{array}$ & $\begin{array}{l}2 \\
9 \\
2\end{array}$ & $\begin{array}{l}\text { PURCHASING } \\
\text { PURELY } \\
\text { PURPORT }\end{array}$ \\
\hline $\begin{array}{r}2 \\
\dot{5} \\
17\end{array}$ & $\begin{array}{l}\text { PRESENTING } \\
\text { PRESENTS } \\
\text { PRESIDENT }\end{array}$ & $\begin{array}{r}3 \\
16 \\
3\end{array}$ & $\begin{array}{l}\text { PROGRESSIVE } \\
\text { PROJECT } \\
\text { PROJECTOR }\end{array}$ & $\begin{array}{l}7 \\
3 \\
3\end{array}$ & $\begin{array}{l}\text { PURPOSE } \\
\text { PURPOSES } \\
\text { PURSE }\end{array}$ \\
\hline $\begin{array}{r}26 \\
3 \\
2\end{array}$ & $\begin{array}{l}\text { PRESS } \\
\text { PRESSES } \\
\text { PRESSING }\end{array}$ & $\begin{array}{l}4 \\
3 \\
8\end{array}$ & $\begin{array}{l}\text { PROJECTORS } \\
\text { PROJECTS } \\
\text { PROLOGUE }\end{array}$ & $\begin{array}{l}3 \\
3 \\
2\end{array}$ & $\begin{array}{l}\text { PUSH } \\
\text { PUSHED } \\
\text { PUSHING }\end{array}$ \\
\hline 9 & PRESSURE & 2 & PROM & 158 & PUT \\
\hline $\begin{array}{r}29 \\
4\end{array}$ & $\begin{array}{l}\text { PRESUMABLY } \\
\text { PRESUME }\end{array}$ & $\begin{array}{l}3 \\
2\end{array}$ & $\begin{array}{l}\text { PROMISED } \\
\text { PROMISES }\end{array}$ & $\begin{array}{r}8 \\
31\end{array}$ & $\begin{array}{l}\text { PUTS } \\
\text { PUTTING }\end{array}$ \\
\hline $\begin{array}{r}5 \\
4 \\
71\end{array}$ & $\begin{array}{l}\text { PRETEND } \\
\text { PRETENDING } \\
\text { PRETTY }\end{array}$ & $\begin{array}{l}3 \\
5 \\
2\end{array}$ & $\begin{array}{l}\text { PROMOTION } \\
\text { PROMPT } \\
\text { PROMPTS }\end{array}$ & $\begin{array}{l}2 \\
9 \\
2\end{array}$ & $\begin{array}{l}\text { PUZZLED } \\
\text { QUALIFIED } \\
\text { QUALIFY }\end{array}$ \\
\hline $\begin{array}{l}2 \\
7 \\
2\end{array}$ & $\begin{array}{l}\text { PREVENT } \\
\text { PREVIOUS } \\
\text { PREVIOUSLY }\end{array}$ & $\begin{array}{l}3 \\
2 \\
9\end{array}$ & $\begin{array}{l}\text { PRONUNCIATION } \\
\text { PROPAGANDA } \\
\text { PROPER }\end{array}$ & $\begin{array}{r}2 \\
10 \\
2\end{array}$ & $\begin{array}{l}\text { QUALITIES } \\
\text { QUALITY } \\
\text { QUARRY }\end{array}$ \\
\hline $\begin{array}{r}19 \\
3 \\
7\end{array}$ & $\begin{array}{l}\text { PRICE } \\
\text { PRICED } \\
\text { PRICES }\end{array}$ & $\begin{array}{l}5 \\
2 \\
2\end{array}$ & $\begin{array}{l}\text { PROPERLY } \\
\text { PROPHECY } \\
\text { PROPORTION }\end{array}$ & $\begin{array}{r}2 \\
14 \\
4\end{array}$ & $\begin{array}{l}\text { QUART } \\
\text { QUARTER } \\
\text { QUARTETS }\end{array}$ \\
\hline 2 & PRIDE & 3 & PROPORTIONS & 2 & QUEEN \\
\hline $\begin{array}{l}2 \\
6\end{array}$ & $\begin{array}{l}\text { PRIEST } \\
\text { PRIMARILY }\end{array}$ & $\begin{array}{l}5 \\
2\end{array}$ & $\begin{array}{l}\text { PROPOSAL } \\
\text { PROPOSALS }\end{array}$ & $\begin{array}{l}3 \\
2\end{array}$ & $\begin{array}{l}\text { QUEEN`S } \\
\text { QUERY }\end{array}$ \\
\hline $\begin{array}{l}3 \\
3 \\
2\end{array}$ & $\begin{array}{l}\text { PRIMARY } \\
\text { PRIME } \\
\text { PRIMITIVE }\end{array}$ & $\begin{array}{l}7 \\
2 \\
3\end{array}$ & $\begin{array}{l}\text { PROPOSE } \\
\text { PROPOSED } \\
\text { PROSE }\end{array}$ & $\begin{array}{r}71 \\
37 \\
6\end{array}$ & $\begin{array}{l}\text { QUEST ION } \\
\text { QUEST IONS } \\
\text { QUEUE }\end{array}$ \\
\hline $\begin{array}{l}2 \\
6 \\
3\end{array}$ & $\begin{array}{l}\text { PRINCESS } \\
\text { PRINCIPAL } \\
\text { PRINCIPALLY }\end{array}$ & $\begin{array}{l}3 \\
4 \\
7\end{array}$ & $\begin{array}{l}\text { PROSPEROUS } \\
\text { PROTECT } \\
\text { PROTESTANT }\end{array}$ & $\begin{array}{r}8 \\
3 \\
12\end{array}$ & $\begin{array}{l}\text { QUICK } \\
\text { QUICKER } \\
\text { QUICKLY }\end{array}$ \\
\hline $\begin{array}{l}5 \\
16 \\
10\end{array}$ & $\begin{array}{l}\text { PRINCIPLE } \\
\text { PRINT } \\
\text { PRINTED }\end{array}$ & $\begin{array}{l}5 \\
3 \\
7\end{array}$ & $\begin{array}{l}\text { PROTESTANTS } \\
\text { PROUD } \\
\text { PROVE }\end{array}$ & $\begin{array}{r}13 \\
18 \\
6\end{array}$ & $\begin{array}{l}\text { QUID } \\
\text { QUIET } \\
\text { QUIETLY }\end{array}$ \\
\hline
\end{tabular}


Appendix A (Continued)

\begin{tabular}{|c|c|c|c|c|c|}
\hline $\begin{array}{l}4 \\
2\end{array}$ & $\begin{array}{l}\text { QUIRKS } \\
\text { QUIRKY }\end{array}$ & $\begin{array}{r}11 \\
6\end{array}$ & $\begin{array}{l}\text { RECOGNIZE } \\
\text { RECOGNIZED }\end{array}$ & $\begin{array}{l}2 \\
5\end{array}$ & $\begin{array}{l}\text { REMAIN ING } \\
\text { REMARK }\end{array}$ \\
\hline $\begin{array}{r}464 \\
2 \\
5\end{array}$ & $\begin{array}{l}\text { QUITE } \\
\text { R } \\
\text { RACE }\end{array}$ & $\begin{array}{l}2 \\
4 \\
4\end{array}$ & $\begin{array}{l}\text { RECOMMEND } \\
\text { RECOMMENDAT ION } \\
\text { RECOMMENDED }\end{array}$ & $\begin{array}{r}2 \\
6 \\
139\end{array}$ & $\begin{array}{l}\text { REMARKABLE } \\
\text { REMARKS } \\
\text { REMEMBER }\end{array}$ \\
\hline 2 & RADICALLY & 2 & RECONCILED & 7 & REMEMBERED \\
\hline $\begin{array}{l}15 \\
13\end{array}$ & $\begin{array}{l}\text { RADIO } \\
\text { RAILWAY }\end{array}$ & $\begin{array}{l}15 \\
10\end{array}$ & $\begin{array}{l}\text { RECORD } \\
\text { RECORDED }\end{array}$ & $\begin{array}{l}3 \\
3\end{array}$ & $\begin{array}{l}\text { REMEMBERING } \\
\text { REMIND }\end{array}$ \\
\hline $\begin{array}{l}4 \\
5 \\
7\end{array}$ & $\begin{array}{l}\text { RAILWAYS } \\
\text { RAIN } \\
\text { RAINBOW }\end{array}$ & $\begin{array}{l}5 \\
3 \\
4\end{array}$ & $\begin{array}{l}\text { RECORDER } \\
\text { RECORDING } \\
\text { RECORDS }\end{array}$ & $\begin{array}{l}2 \\
4 \\
2\end{array}$ & $\begin{array}{l}\text { REMINDED } \\
\text { REMINDS } \\
\text { REMINISCING }\end{array}$ \\
\hline $\begin{array}{l}6 \\
4 \\
5\end{array}$ & $\begin{array}{l}\text { RAINFALL } \\
\text { RAINING } \\
\text { RAISE }\end{array}$ & $\begin{array}{l}4 \\
9 \\
2\end{array}$ & $\begin{array}{l}\text { RECOVER } \\
\text { RED } \\
\text { REDI STRIBUTE }\end{array}$ & $\begin{array}{l}2 \\
6 \\
6\end{array}$ & $\begin{array}{l}\text { REMOTE } \\
\text { REMOVE } \\
\text { REMOVED }\end{array}$ \\
\hline 8 & RAISED & 2 & REEL & 15 & RENAISSANCE \\
\hline 2 & RAMPS & 3 & REELS & 6 & RENAL \\
\hline 6 & RAN & 3 & REFECTORY & 2 & RENEWAL \\
\hline 26 & RANG & 3 & REFER & 4 & RENT \\
\hline 13 & RANGE & 2 & REFEREE & 3 & REPERCUSSIONS \\
\hline 4 & RANKS & 2 & REFEREES & 2 & REPLACE \\
\hline $\begin{array}{l}3 \\
9 \\
2\end{array}$ & $\begin{array}{l}\text { RAPIDLY } \\
\text { RARE } \\
\text { RAT }\end{array}$ & $\begin{array}{l}4 \\
6 \\
3\end{array}$ & $\begin{array}{l}\text { REFERENCE } \\
\text { REFERENCES } \\
\text { REFERENDUM }\end{array}$ & $\begin{array}{l}2 \\
4 \\
2\end{array}$ & $\begin{array}{l}\text { REPLACEMENT } \\
\text { REPLIED } \\
\text { REPLY }\end{array}$ \\
\hline 3 & RATA & 3 & REFERRED & 12 & REPORT \\
\hline $\begin{array}{r}17 \\
219\end{array}$ & $\begin{array}{l}\text { RATE } \\
\text { RATHER }\end{array}$ & $\begin{array}{l}3 \\
4\end{array}$ & $\begin{array}{l}\text { REFERRING } \\
\text { REFLECTION }\end{array}$ & $\begin{array}{l}2 \\
2\end{array}$ & $\begin{array}{l}\text { REPORTED } \\
\text { REPORTS }\end{array}$ \\
\hline 2 & RATIO & 2 & REFLECTS & 2 & REPRESENT \\
\hline $\begin{array}{l}4 \\
6\end{array}$ & $\begin{array}{l}\text { RAYON } \\
\text { REACH }\end{array}$ & $\begin{array}{l}2 \\
2\end{array}$ & $\begin{array}{l}\text { REFORMED } \\
\text { REFORMS }\end{array}$ & $\begin{array}{l}3 \\
2\end{array}$ & $\begin{array}{l}\text { REPRESENTATIVE } \\
\text { REPRESENTATIVES }\end{array}$ \\
\hline $\begin{array}{r}4 \\
7 \\
238\end{array}$ & $\begin{array}{l}\text { REACHED } \\
\text { REACTION } \\
\text { READ }\end{array}$ & $\begin{array}{l}7 \\
7 \\
3\end{array}$ & $\begin{array}{l}\text { REFUSED } \\
\text { REGARD } \\
\text { REGARDS }\end{array}$ & $\begin{array}{l}2 \\
4 \\
3\end{array}$ & $\begin{array}{l}\text { REPRESENTED } \\
\text { REPRINT } \\
\text { REPRINTS }\end{array}$ \\
\hline 8 & READER & 3 & REGIME & 3 & REPRODUCE \\
\hline $\begin{array}{r}2 \\
71\end{array}$ & $\begin{array}{l}\text { READERSH IP } \\
\text { READING }\end{array}$ & $\begin{array}{l}2 \\
3\end{array}$ & $\begin{array}{l}\text { REGIMENT } \\
\text { REGISTRAR }\end{array}$ & $\begin{array}{l}2 \\
5\end{array}$ & $\begin{array}{l}\text { REPUBLIC } \\
\text { REPUTATION }\end{array}$ \\
\hline 16 & READY & 2 & REGULAR & 3 & REQUIRE \\
\hline 27 & REAL & 7 & REGULARLY & 7 & REQUIRED \\
\hline 2 & REALIST & 3 & REGULAT ION & 3 & REQUIRES \\
\hline 4 & REALISTIC & 5 & REGULATIONS & 2 & REQUIRING \\
\hline $\begin{array}{l}2 \\
6\end{array}$ & $\begin{array}{l}\text { REALIST ICALLY } \\
\text { REALITY }\end{array}$ & $\begin{array}{l}2 \\
2\end{array}$ & $\begin{array}{l}\text { REIGN } \\
\text { REINFORCEMENTS }\end{array}$ & $\begin{array}{r}70 \\
3\end{array}$ & $\begin{array}{l}\text { RESEARCH } \\
\text { RESERVATIONS }\end{array}$ \\
\hline 29 & REALIZE & 2 & RELATE & 2 & RESERVE \\
\hline 18 & REALIZED & 8 & RELATED & 4 & RESERVED \\
\hline 3 & REALIZING & 2 & RELATING & 2 & RESIDENCE \\
\hline 633 & REALLY & 7 & RELATION & 2 & RESIDENTIAL \\
\hline 2 & REAMS & 8 & RELATIONS & 3 & RESIGN \\
\hline 35 & REASON & 8 & RELAT IONSHIP & 2 & RESISTANCE \\
\hline $\begin{array}{r}11 \\
8 \\
10\end{array}$ & $\begin{array}{l}\text { REASONABLE } \\
\text { REASONABLY } \\
\text { REASONS }\end{array}$ & $\begin{array}{l}2 \\
5 \\
3\end{array}$ & $\begin{array}{l}\text { RELATIVE } \\
\text { RELATIVELY } \\
\text { RELAX }\end{array}$ & $\begin{array}{l}2 \\
4 \\
6\end{array}$ & $\begin{array}{l}\text { RESISTED } \\
\text { RESIT } \\
\text { RESOURCES }\end{array}$ \\
\hline 2 & REBUILT & 10 & RELAXED & 9 & RESPECT \\
\hline $\begin{array}{l}2 \\
2\end{array}$ & $\begin{array}{l}\text { RECALL } \\
\text { RECEIVED }\end{array}$ & $\begin{array}{l}2 \\
2\end{array}$ & $\begin{array}{l}\text { RELEASE } \\
\text { RELEVANT }\end{array}$ & $\begin{array}{l}4 \\
5\end{array}$ & $\begin{array}{l}\text { RESPECTABLE } \\
\text { RESPECTS }\end{array}$ \\
\hline $\begin{array}{r}15 \\
26 \\
2\end{array}$ & $\begin{array}{l}\text { RECENT } \\
\text { RECENTLY } \\
\text { RECESS }\end{array}$ & $\begin{array}{l}2 \\
2 \\
3\end{array}$ & $\begin{array}{l}\text { RELIGION } \\
\text { RELIGIONS } \\
\text { RELUCTANT }\end{array}$ & $\begin{array}{r}4 \\
3 \\
10\end{array}$ & $\begin{array}{l}\text { RESPOND } \\
\text { RESPONSE } \\
\text { RESPONSIBILITY }\end{array}$ \\
\hline 9 & RECKON & 5 & RELY & 7 & RESPONSIBLE \\
\hline $\begin{array}{l}3 \\
2\end{array}$ & $\begin{array}{l}\text { RECKONED } \\
\text { RECKONS }\end{array}$ & $\begin{array}{l}2 \\
2\end{array}$ & $\begin{array}{l}\text { REMA IN } \\
\text { REMA I NDER }\end{array}$ & $\begin{array}{r}42 \\
3\end{array}$ & $\begin{array}{l}\text { REST } \\
\text { RESTAURANTS }\end{array}$ \\
\hline 6 & RECOGNITION & 4 & REMAINED & 4 & RESTORATION \\
\hline
\end{tabular}


Appendix A (Continued)

\begin{tabular}{|c|c|c|c|c|c|}
\hline 3 & RESTRICTED & 2 & ROUBLES & 2 & SCARED \\
\hline $\begin{array}{r}11 \\
6 \\
4\end{array}$ & $\begin{array}{l}\text { RESULT } \\
\text { RESULTS } \\
\text { RETIRE }\end{array}$ & $\begin{array}{r}2 \\
6 \\
139\end{array}$ & $\begin{array}{l}\text { ROUGH } \\
\text { ROUGHLY } \\
\text { ROUND }\end{array}$ & $\begin{array}{r}2 \\
2 \frac{1}{2}\end{array}$ & $\begin{array}{l}\text { SCATTERED } \\
\text { SCENE } \\
\text { SCENES }\end{array}$ \\
\hline 4 & RETIRED & 2 & ROUNDABOUT & 2 & SCEPTICAL \\
\hline $\begin{array}{l}2 \\
5\end{array}$ & $\begin{array}{l}\text { RETIRES } \\
\text { RETURN }\end{array}$ & $\begin{array}{l}2 \\
5\end{array}$ & $\begin{array}{l}\text { ROUTE } \\
\text { ROUTINE }\end{array}$ & $\begin{array}{r}10 \\
2\end{array}$ & $\begin{array}{l}\text { SCHEME } \\
\text { SCHEMES }\end{array}$ \\
\hline $\begin{array}{l}2 \\
2 \\
2\end{array}$ & $\begin{array}{l}\text { REVEALING } \\
\text { REVELATION } \\
\text { REVEREND }\end{array}$ & $\begin{array}{l}7 \\
8 \\
2\end{array}$ & $\begin{array}{l}\text { ROW } \\
\text { ROYAL } \\
\text { RP }\end{array}$ & $\begin{array}{l}2 \\
5 \\
6\end{array}$ & $\begin{array}{l}\text { SCHOLAR } \\
\text { SCHOLARLY } \\
\text { SCHOLARSHIP }\end{array}$ \\
\hline $\begin{array}{l}2 \\
2 \\
3\end{array}$ & $\begin{array}{l}\text { REVERSE } \\
\text { REVERT } \\
\text { REVIEW }\end{array}$ & $\begin{array}{l}2 \\
2 \\
2\end{array}$ & $\begin{array}{l}\text { RS } \\
\text { RUB } \\
\text { RUBBING }\end{array}$ & $\begin{array}{l}87 \\
10 \\
15\end{array}$ & $\begin{array}{l}\text { SCHOOL } \\
\text { SCHOOLS } \\
\text { SCIENCE }\end{array}$ \\
\hline $\begin{array}{l}4 \\
5 \\
3\end{array}$ & $\begin{array}{l}\text { REVI EWS } \\
\text { REVISED } \\
\text { REVISING }\end{array}$ & $\begin{array}{r}10 \\
4 \\
8\end{array}$ & $\begin{array}{l}\text { RUBBISH } \\
\text { RUDE } \\
\text { RULE }\end{array}$ & $\begin{array}{l}4 \\
3 \\
2\end{array}$ & $\begin{array}{l}\text { SCIENTIFIC } \\
\text { SCIENTIST } \\
\text { SCIENTISTS }\end{array}$ \\
\hline $\begin{array}{l}2 \\
2 \\
5\end{array}$ & $\begin{array}{l}\text { REVISION } \\
\text { REVOLUTION } \\
\text { RHEINGOLD }\end{array}$ & $\begin{array}{r}8 \\
22 \\
5\end{array}$ & $\begin{array}{l}\text { RULES } \\
R U N \\
R U N G\end{array}$ & $\begin{array}{l}2 \\
3 \\
4\end{array}$ & $\begin{array}{l}\text { SCOPE } \\
\text { SCOTCH } \\
\text { SCRATCH }\end{array}$ \\
\hline $\begin{array}{l}2 \\
2 \\
7\end{array}$ & $\begin{array}{l}\text { RHYMING } \\
\text { RHYTHM } \\
\text { RICH }\end{array}$ & $\begin{array}{r}19 \\
2 \\
6\end{array}$ & $\begin{array}{l}\text { RUNNING } \\
\text { RUNS } \\
\text { RUSH }\end{array}$ & $\begin{array}{l}3 \\
4 \\
3\end{array}$ & $\begin{array}{l}\text { SCRATCHED } \\
\text { SCREEN } \\
\text { SCREENS }\end{array}$ \\
\hline $\begin{array}{r}17 \\
11 \\
7\end{array}$ & $\begin{array}{l}\text { RICKETS } \\
\text { RID } \\
\text { RIDICULOUS }\end{array}$ & $\begin{array}{r}2 \\
4 \\
17\end{array}$ & $\begin{array}{l}\text { RUSHING } \\
\text { RUSTICS } \\
\text { S }\end{array}$ & $\begin{array}{l}4 \\
7 \\
4\end{array}$ & $\begin{array}{l}\text { SCRIPT } \\
\text { SCRIPTS } \\
\text { SCULPTURE }\end{array}$ \\
\hline $\begin{array}{r}2 \\
372 \\
3\end{array}$ & $\begin{array}{l}\text { RIDING } \\
\text { RIGHT } \\
\text { RIGHTHAND }\end{array}$ & $\begin{array}{l}6 \\
5 \\
3\end{array}$ & $\begin{array}{l}\text { SABBATICAL } \\
\text { SACK } \\
\text { SACKING }\end{array}$ & $\begin{array}{r}3 \\
13 \\
3\end{array}$ & $\begin{array}{l}\text { SCURVY } \\
\text { SEA } \\
\text { SEASON }\end{array}$ \\
\hline $\begin{array}{l}3 \\
3 \\
2\end{array}$ & $\begin{array}{l}\text { RIGHTLY } \\
\text { RIGID } \\
\text { RINDAN }\end{array}$ & $\begin{array}{l}7 \\
2 \\
2\end{array}$ & $\begin{array}{l}\text { SAD } \\
\text { SAFARI } \\
\text { SAFE }\end{array}$ & $\begin{array}{r}3 \\
10 \\
5\end{array}$ & $\begin{array}{l}\text { SEAT } \\
\text { SEATS } \\
\text { SEBACEOUS }\end{array}$ \\
\hline $\begin{array}{r}21 \\
2 \\
2\end{array}$ & $\begin{array}{l}\text { RING } \\
\text { RINGED } \\
\text { RINGING }\end{array}$ & $\begin{array}{r}4 \\
568 \\
2\end{array}$ & $\begin{array}{l}\text { SAFER } \\
\text { SAID } \\
\text { SAILED }\end{array}$ & $\begin{array}{r}59 \\
3 \\
5\end{array}$ & $\begin{array}{l}\text { SECOND } \\
\text { SECONDLY } \\
\text { SECONDS }\end{array}$ \\
\hline $\begin{array}{l}8 \\
3 \\
2\end{array}$ & $\begin{array}{l}\text { RINGS } \\
\text { RIP } \\
\text { RIPE }\end{array}$ & $\begin{array}{l}2 \\
4 \\
6\end{array}$ & $\begin{array}{l}\text { SAILORS } \\
\text { SAKE } \\
\text { SALARY }\end{array}$ & $\begin{array}{r}3 \\
3 \\
10\end{array}$ & $\begin{array}{l}\text { SECRET } \\
\text { SECRETARIAL } \\
\text { SECRETARIES }\end{array}$ \\
\hline $\begin{array}{r}11 \\
6 \\
14\end{array}$ & $\begin{array}{l}\text { RISE } \\
\text { RISES } \\
\text { RISK }\end{array}$ & $\begin{array}{r}2 \\
136 \\
2\end{array}$ & $\begin{array}{l}\text { SALES } \\
\text { SAME } \\
\text { SAND }\end{array}$ & $\begin{array}{r}12 \\
2 \\
18\end{array}$ & $\begin{array}{l}\text { SECRETARY } \\
\text { SECRETLY } \\
\text { SECTION }\end{array}$ \\
\hline $\begin{array}{l}3 \\
4 \\
3\end{array}$ & $\begin{array}{l}\text { RISKS } \\
\text { RIVER } \\
\text { RIVERS }\end{array}$ & $\begin{array}{r}2 \\
15 \\
5\end{array}$ & $\begin{array}{l}\text { SARCASM } \\
\text { SAT } \\
\text { SATIRE }\end{array}$ & $\begin{array}{r}2 \\
2 \\
2\end{array}$ & $\begin{array}{l}\text { SECTIONS } \\
\text { SECURE } \\
\text { SECURITIES }\end{array}$ \\
\hline $\begin{array}{r}34 \\
5 \\
2\end{array}$ & $\begin{array}{l}\text { ROAD } \\
\text { ROADS } \\
\text { ROCK }\end{array}$ & $\begin{array}{l}2 \\
8 \\
2\end{array}$ & $\begin{array}{l}\text { SATIRICAL } \\
\text { SATISFACTORY } \\
\text { SAT I SF IED }\end{array}$ & $\begin{array}{r}796 \\
3 \\
2\end{array}$ & $\begin{array}{l}\text { SEE } \\
\text { SEEDS } \\
\text { SEEDY }\end{array}$ \\
\hline $\begin{array}{l}2 \\
2 \\
3\end{array}$ & $\begin{array}{l}\text { ROCKS } \\
\text { RODEOS } \\
\text { ROLLING }\end{array}$ & $\begin{array}{r}11 \\
5 \\
3\end{array}$ & $\begin{array}{l}\text { SATURDAY } \\
\text { SATURDAYS } \\
\text { SAUCEPANS }\end{array}$ & $\begin{array}{l}22 \\
48 \\
28\end{array}$ & $\begin{array}{l}\text { SEEING } \\
\text { SEEM } \\
\text { SEEMED }\end{array}$ \\
\hline $\begin{array}{r}11 \\
2 \\
4\end{array}$ & $\begin{array}{l}\text { ROMAN } \\
\text { ROMANCE } \\
\text { ROMANCES }\end{array}$ & $\begin{array}{l}3 \\
9 \\
2\end{array}$ & $\begin{array}{l}\text { SAUCERS } \\
\text { SAVE } \\
\text { SAVED }\end{array}$ & $\begin{array}{r}60 \\
73 \\
6\end{array}$ & $\begin{array}{l}\text { SEEMS } \\
\text { SEEN } \\
\text { SEES }\end{array}$ \\
\hline $\begin{array}{r}4 \\
2 \\
89\end{array}$ & $\begin{array}{l}\text { ROMANTIC } \\
\text { ROMANTICS } \\
\text { ROOM }\end{array}$ & $\begin{array}{r}2 \\
2 \\
41\end{array}$ & $\begin{array}{l}\text { SAVES } \\
\text { SAVINGS } \\
\text { SAW }\end{array}$ & $\begin{array}{l}2 \\
2 \\
6\end{array}$ & $\begin{array}{l}\text { SEIZE } \\
\text { SELECT } \\
\text { SELECTED }\end{array}$ \\
\hline $\begin{array}{r}19 \\
3 \\
2\end{array}$ & $\begin{array}{l}\text { ROOMS } \\
\text { ROOTS } \\
\text { ROSE }\end{array}$ & $\begin{array}{l}432 \\
112 \\
112\end{array}$ & $\begin{array}{l}\text { SAY } \\
\text { SAYING } \\
\text { SAYS }\end{array}$ & $\begin{array}{l}3 \\
2 \\
2\end{array}$ & $\begin{array}{l}\text { SELECTION } \\
\text { SELECTIONS } \\
\text { SELECTIVE }\end{array}$ \\
\hline $\begin{array}{l}3 \\
4\end{array}$ & $\begin{array}{l}\text { ROTTEN } \\
\text { ROUBLE }\end{array}$ & $\begin{array}{r}10 \\
2\end{array}$ & $\begin{array}{l}\text { SCALE } \\
\text { SCARCELY }\end{array}$ & $\begin{array}{l}3 \\
4\end{array}$ & $\begin{array}{l}\text { SELF } \\
\text { SELFCONTAINED }\end{array}$ \\
\hline
\end{tabular}


Appendix A (Continued)

\begin{tabular}{|c|c|c|c|c|c|}
\hline 3 & SELFEVIDENT & 9 & SHAPE & 11 & SIGNS \\
\hline 2 & SELF PERPETUAT ING & 7 & SHAPED & 2 & SILENT \\
\hline 18 & SELL & 18 & SHARE & 24 & SILLY \\
\hline 3 & SELLING & 4 & SHARED & 18 & SIMILAR \\
\hline 3 & SELLS & 14 & SHARES & 2 & SIMILARITIES \\
\hline 20 & SEMINAR & 11 & SHARP & 13 & \\
\hline 2 & SEMI NARROOM & 5 & SHARPLY & 2 & $\begin{array}{l}\text { S IMP LER } \\
\text { STMP I F ST }\end{array}$ \\
\hline 3 & SEMI NARROOMS & 486 & SHE & 2 & S IMP LEST \\
\hline 18 & SEMINARS & 3 & SHED & 34 & S IMP LY \\
\hline 8 & SENATE & 10 & SHEER & 2 & SIMULTANEOU SLY \\
\hline 25 & SEND & 2 & SHEIKHS & 3 & SIN \\
\hline 8 & SENDING & 5 & SHELF & 86 & SINCE \\
\hline 3 & SENDS & 2 & SHELVES & 2 & SING \\
\hline 11 & SENIOR & 13 & SHERRY & 12 & SINGING \\
\hline 63 & SENSE & 33 & $S H E^{`} D$ & 8 & SINGLE \\
\hline 9 & SENSIBLE & 4 & SHE LL & 2 & SINGULAR \\
\hline 2 & SENSITIVE & 124 & SHE'S & 4 & SINK \\
\hline 20 & SENT & 2 & SHIFT & 37 & SIR \\
\hline 12 & SENTENCE & 2 & SHIFTED & 13 & SISTER \\
\hline 4 & SENTENCES & 2 & SHIETS & 39 & SIT \\
\hline 15 & SEPARATE & 2 & SHILLINGS & 8 & SITE \\
\hline 2 & SEPARATED & 2 & SHINGLES & 4 & SITES \\
\hline 11 & SEPTEMBER & 3 & SHIP & 5 & SITS \\
\hline 2 & SEQUENCE & 5 & SHIRT & 27 & SITTING \\
\hline 2 & SERGEANT & 3 & SHIT & 2 & SITUATED \\
\hline 21 & SERIES & 3 & SHOCK & 26 & SITUATION \\
\hline 17 & SERIOUS & 3 & SHOES & 6 & SITUATIONS \\
\hline 17 & SERIOUSLY & 5 & SHOOT & 71 & SIX \\
\hline 2 & SERVANT & 11 & SHOP & 2 & SIXPENCE \\
\hline 2 & SERVANTS & 8 & SHOPP ING & 20 & S IXTEEN \\
\hline 2 & SERVE & 4 & SHOPS & 8 & SIXTH \\
\hline 18 & SERVICE & 27 & SHOR'T & 2 & SIXTIES \\
\hline 5 & SERVICES & 5 & SHORTAGE & 17 & SIXTY \\
\hline 2 & SESSION & 2 & SHORTER & 3 & SIXTYEIGHT \\
\hline 2 & SESSIONS & 2 & SHORTLY & 6 & SIXTYF IVE \\
\hline 77 & SET & 2 & SHORTSIGHTED & 4 & SIXT YNINE \\
\hline 7 & SETS & 2 & SHORTTERM & 2 & SIXTYONE \\
\hline 10 & SETT ING & 8 & SHOT & 4 & SIXTYSIX \\
\hline 3 & SETTLE & 2 & SHOTGUN & 12 & SIZE \\
\hline 4 & SETTLED & 175 & SHOULD & 2 & SKETCH \\
\hline 9 & SETUP & 31 & SHOU LDN'T & 2 & SKIING \\
\hline 53 & SEVEN & 2 & SHOUT ING & 3 & SKILL \\
\hline 9 & SEVENTEEN & 3 & SHOVE & 3 & SKILLS \\
\hline 4 & SEVENTEENTH & 6 & SHOVED & 3 & SKIN \\
\hline 3 & SEVENTH & 27 & SHOW & 2 & SKY \\
\hline 7 & SEVENTY & 8 & SHOWED & 2 & SLACK \\
\hline 3 & SEVENTYEIGHT & 5 & SHOWING & 7 & SLEEP \\
\hline 2 & SEVENTYFIVE & 6 & SHOWN & 3 & SLEEP ING \\
\hline 4 & SEVENTYSIX & 6 & SHOWS & 2 & SLIDE \\
\hline 3 & SEVENTYTWO & 6 & SHUT & 7 & SLIDES \\
\hline 22 & SEVERAL & 2 & SHY & 5 & SLIGHT \\
\hline 2 & SEW & 4 & SICK & 7 & SLIGHTEST \\
\hline $\begin{array}{l}5 \\
8\end{array}$ & $\begin{array}{l}\text { SEWI NGMACHINE } \\
\text { SEX }\end{array}$ & $\begin{array}{r}68 \\
5\end{array}$ & $\begin{array}{l}\text { SIDE } \\
\text { SIDES }\end{array}$ & $\begin{array}{r}26 \\
2\end{array}$ & $\begin{array}{l}\text { SLIGHTLY } \\
\text { SLIM }\end{array}$ \\
\hline 2 & SHAKEN & 4 & SIDEWAYS & 4 & SLIP \\
\hline 2 & SHAKES & 10 & SIGHT & 3 & SLIPS \\
\hline 2 & SHAK ING & 26 & SIGN & 2 & SLOT \\
\hline 72 & SHALL & 3 & SIGNAL & 4 & SLOW \\
\hline 7 & SHAME & 2 & SIGNALMAN & 3 & SLOWER \\
\hline 4 & SHAN $^{\top} \mathrm{T}$ & 5 & SIGNED & 5 & SLOWLY \\
\hline
\end{tabular}


Appendix A (Continued)

\begin{tabular}{|c|c|c|c|c|c|}
\hline $\begin{array}{l}3 \\
2\end{array}$ & $\begin{array}{l}\text { SMACK } \\
\text { SMALE }\end{array}$ & $\begin{array}{l}10 \\
34\end{array}$ & $\begin{array}{l}\text { SOUNDED } \\
\text { SOUNDS }\end{array}$ & $\begin{array}{l}8 \\
2\end{array}$ & $\begin{array}{l}\text { STAIRS } \\
\text { STALK }\end{array}$ \\
\hline 47 & SMALL & 2 & SOUP & 29 & STAND \\
\hline 5 & SMALLER & 4 & SOURCE & 14 & STANDARD \\
\hline 2 & SMALLEST & 4 & SOURCES & 6 & STANDARDS \\
\hline 2 & SMART & 49 & SOUTH & 7 & STANDING \\
\hline 4 & SMASHED & 2 & SOUTHEAST & 9 & STANDS \\
\hline 2 & SMATTERING & 4 & SOUTHERN & 2 & STAR \\
\hline 4 & SMILE & 2 & $\mathrm{so}^{\circ} \mathrm{S}$ & 2 & STARS \\
\hline 6 & SMOKE & 9 & SPACE & 66 & START \\
\hline 2 & SMOKES & 8 & SPARE & 65 & STARTED \\
\hline 5 & SMOKING & 3 & SPASTIC & 2 & STARTER \\
\hline $\begin{array}{l}3 \\
2\end{array}$ & $\begin{array}{l}\text { SMOOTH } \\
\text { SNIDE }\end{array}$ & $\begin{array}{r}3 \\
35\end{array}$ & $\begin{array}{l}\text { SPASTICS } \\
\text { SPEAK }\end{array}$ & $\begin{array}{l}10 \\
20\end{array}$ & $\begin{array}{l}\text { START ING } \\
\text { STARTS }\end{array}$ \\
\hline 3 & SNOBS & 4 & SPEAKER & 11 & STATE \\
\hline 2 & SNOW & 6 & SPEAKERS & 2 & STATELY \\
\hline 1188 & So & 18 & SPEAKING & 5 & STATEMENT \\
\hline 2 & $\mathrm{SOB}$ & 5 & SPEAKS & 2 & STATEMENTS \\
\hline 3 & SOCALLED & 29 & SPECIAL & 2 & STATESMAN \\
\hline 16 & SOCIAL & 6 & SPECIALIST & 2 & STATIC \\
\hline 10 & SOCIETY & 3 & SPECIALISTS & 6 & STATION \\
\hline $\begin{array}{l}2 \\
3\end{array}$ & $\begin{array}{l}\text { SOCIOLOGY } \\
\text { SOCKS }\end{array}$ & $\begin{array}{l}2 \\
2\end{array}$ & $\begin{array}{l}\text { SPECIALITY } \\
\text { SPECIALLY }\end{array}$ & $\begin{array}{l}4 \\
2\end{array}$ & $\begin{array}{l}\text { STATIONS } \\
\text { STATISTICAL }\end{array}$ \\
\hline 2 & SOD & 3 & SPECIALS & 4 & STATISTICS \\
\hline 2 & SOFA & 7 & SPECIFIC & 2 & STATUES \\
\hline 5 & SOFT & 4 & SPECIFICALLY & 13 & STATUS \\
\hline 11 & SOLD & 14 & SPEECH & 38 & STAY \\
\hline 3 & SOLDIER & 2 & SPEECHES & 13 & STAYED \\
\hline 4 & SOLDIERS & 3 & SPEED & 6 & STAYING \\
\hline 2 & SOLE & 10 & SPELL & 3 & STAYS \\
\hline $\begin{array}{l}3 \\
2\end{array}$ & SOLELY & $\begin{array}{r}3 \\
27\end{array}$ & $\begin{array}{l}\text { SPELLING } \\
\text { SPEND }\end{array}$ & $\begin{array}{l}3 \\
3\end{array}$ & $\begin{array}{l}\text { STC } \\
\text { STEADY }\end{array}$ \\
\hline $\begin{array}{l}2 \\
9\end{array}$ & $\begin{array}{l}\text { SOLICIIURS } \\
\text { SOLID }\end{array}$ & 5 & SPENDING & 2 & STEAK \\
\hline 2 & SOLI LOQUY & 27 & SPENT & 2 & STEAL \\
\hline 419 & SOME & 5 & SPIRIT & 2 & STEALS \\
\hline 87 & SOMEBODY & 2 & SPIT & 2 & STEELRIMMED \\
\hline 10 & SOMEBODY'S & 7 & SPITE & 3 & STEMS \\
\hline 10 & SOMEHOW & 8 & SPLENDID & 4 & STEP \\
\hline 26 & SOMEONE & 4 & SPLIT & 4 & STEPS \\
\hline 2 & SOMEONE $\mathrm{S}$ & 2 & SPIITTING & 17 & STICK \\
\hline 341 & SOMETHING & 2 & SPOIL & 6 & STICKING \\
\hline 5 & SOMETIME & 13 & SPOKE & 130 & STILL \\
\hline 32 & SOMETIMES & 8 & SPOKEN & 2 & STIR \\
\hline 2 & SOMEWHAT & 3 & SPOONS & 2 & STITCHES \\
\hline 36 & SOMEWHERE & 11 & SPOT & 4 & STOCK \\
\hline 7 & SON & II & SPREAD & 13 & STOCKBROKER \\
\hline 3 & SONGS & 2 & SPREADING & 2 & STOCKBROKERS \\
\hline 9 & SONS & 6 & SPRING & 3 & STOLE \\
\hline 20 & SOON & 2 & SPRINKLE & 8 & STONE \\
\hline 6 & SOONER & 3 & SPY & 3 & STONES \\
\hline 3 & SOPHISTICATED & 7 & SQUARE & 6 & STOOD \\
\hline 2 & $\begin{array}{l}\text { SOPRANO } \\
\text { SOPRANOS }\end{array}$ & $\begin{array}{l}9 \\
2\end{array}$ & $\begin{array}{l}\text { ST } \\
\text { STABBED }\end{array}$ & $\begin{array}{r}27 \\
7\end{array}$ & $\begin{array}{l}\text { STOP } \\
\text { STOPPED }\end{array}$ \\
\hline $\begin{array}{l}2 \\
2\end{array}$ & $\begin{array}{l}\text { SOPRANOS } \\
\text { SORE }\end{array}$ & 2 & STABLE & 2 & STOPPING \\
\hline $\begin{array}{r}97 \\
663\end{array}$ & $\begin{array}{l}\text { SORRY } \\
\text { SORT }\end{array}$ & $\begin{array}{r}3 \\
53\end{array}$ & $\begin{array}{l}\text { STACKS } \\
\text { STAFF }\end{array}$ & $\begin{array}{l}3 \\
2\end{array}$ & $\begin{array}{l}\text { STOPS } \\
\text { STORE }\end{array}$ \\
\hline 4 & SORTED & 32 & STAGE & 6 & STORIES \\
\hline 28 & SORTS & 2 & STAGGERED & 3 & STORM \\
\hline 7 & SOUL & 3 & STAG ING & 25 & STORY \\
\hline 16 & SOUND & 2 & STAID & 7 & STOUT \\
\hline
\end{tabular}


Appendix A (Continued)

\begin{tabular}{|c|c|c|c|c|c|}
\hline 31 & S'TRAIGHT & 3 & SUGGESTING & 8 & SYMP TOMS \\
\hline 3 & STRAIGHTEORWARD & 9 & SUGGESTION & 2 & SYNTHESIS \\
\hline 27 & STRANGE & 4 & SUI & 29 & SYSTEM \\
\hline 2 & STRANGERS & 3 & SUIT & 2 & SYSTEMATIC \\
\hline $\begin{array}{r}3 \\
34 \\
2\end{array}$ & $\begin{array}{l}\text { STRANGLE } \\
\text { STREET } \\
\text { STREETS }\end{array}$ & $\begin{array}{l}2 \\
4 \\
4\end{array}$ & $\begin{array}{l}\text { SUITABLE } \\
\text { SUITED } \\
\text { SUITS }\end{array}$ & $\begin{array}{r}4 \\
8 \\
19\end{array}$ & $\begin{array}{l}\text { SYSTEMS } \\
\text { T } \\
\text { TABLE }\end{array}$ \\
\hline $\begin{array}{l}6 \\
2 \\
2\end{array}$ & $\begin{array}{l}\text { STRENGTH } \\
\text { STRENGTHENED } \\
\text { STRETCHES }\end{array}$ & $\begin{array}{r}4 \\
7 \\
46\end{array}$ & $\begin{array}{l}\text { SUM } \\
\text { SUMMARY } \\
\text { SUMMER }\end{array}$ & $\begin{array}{l}6 \\
3 \\
3\end{array}$ & $\begin{array}{l}\text { TABLES } \\
\text { TABLETS } \\
\text { TABOO }\end{array}$ \\
\hline 5 & STRICTLY & 3 & SUMS & 2 & TACK \\
\hline $\begin{array}{r}10 \\
2\end{array}$ & $\begin{array}{l}\text { STRIKE } \\
\text { STRIKERS }\end{array}$ & $\begin{array}{l}13 \\
20\end{array}$ & $\begin{array}{l}\text { SUN } \\
\text { SUNDAY }\end{array}$ & $\begin{array}{l}2 \\
4\end{array}$ & $\begin{array}{l}\text { TACKED } \\
\text { TACKLE }\end{array}$ \\
\hline 7 & STRIKES & 2 & SUNDAYS & 2 & TACTFUL \\
\hline $\begin{array}{l}3 \\
3\end{array}$ & $\begin{array}{l}\text { STRING } \\
\text { STRINGS }\end{array}$ & $\begin{array}{r}4 \\
13\end{array}$ & $\begin{array}{l}\text { SUNNY } \\
\text { SUPER }\end{array}$ & $\begin{array}{l}2 \\
3\end{array}$ & $\begin{array}{l}\text { TAGS } \\
\text { TAIL }\end{array}$ \\
\hline $\begin{array}{r}2 \\
26 \\
2\end{array}$ & $\begin{array}{l}\text { STRIPPED } \\
\text { STRONG } \\
\text { STRONGER }\end{array}$ & $\begin{array}{l}6 \\
2 \\
2\end{array}$ & $\begin{array}{l}\text { SUPERB } \\
\text { SUPERFICIAL } \\
\text { SUPERVISE }\end{array}$ & $\begin{array}{r}2 \\
152 \\
37\end{array}$ & $\begin{array}{l}\text { TAILING } \\
\text { TAKE } \\
\text { TAKEN }\end{array}$ \\
\hline $\begin{array}{l}2 \\
6 \\
8\end{array}$ & $\begin{array}{l}\text { STRONGEST } \\
\text { STRONGLY } \\
\text { STRUCK }\end{array}$ & $\begin{array}{l}2 \\
3 \\
7\end{array}$ & $\begin{array}{l}\text { SUPERVISION } \\
\text { SUPERVISOR } \\
\text { SUPPER }\end{array}$ & $\begin{array}{r}23 \\
41 \\
3\end{array}$ & $\begin{array}{l}\text { TAKES } \\
\text { TAKING } \\
\text { TALENT }\end{array}$ \\
\hline 3 & STRUCTURALISM & 2 & SUPPLIED & 5 & TALENTS \\
\hline 8 & $\begin{array}{l}\text { STRUCTURE } \\
\text { STPUCTURFS }\end{array}$ & 9 & SUPPLY & 73 & TALK \\
\hline 2 & STRUCTURES & 13 & $\begin{array}{l}\text { SUPPORT } \\
\text { SUPPOPTFO }\end{array}$ & 20 & TALKED \\
\hline $\begin{array}{l}13 \\
39 \\
53\end{array}$ & $\begin{array}{l}\text { STUCK } \\
\text { STUDENT } \\
\text { STUDENTS }\end{array}$ & $\begin{array}{l}9 \\
2 \\
6\end{array}$ & $\begin{array}{l}\text { SUPPORTED } \\
\text { SUPPORTERS } \\
\text { SUPPORTING }\end{array}$ & $\begin{array}{r}76 \\
8 \\
3\end{array}$ & $\begin{array}{l}\text { TALK ING } \\
\text { TALKS } \\
\text { TALL }\end{array}$ \\
\hline 7 & STUDIED & 150 & SUPPOSE & 2 & TAP \\
\hline 21 & STUDIES & $\begin{array}{r}35 \\
3\end{array}$ & $\begin{array}{l}\text { SUPPOSED } \\
\text { SUPPOSING }\end{array}$ & $1 \frac{1}{2}$ & $\begin{array}{l}\text { TAPE } \\
\text { TAPFRFCORDFRS }\end{array}$ \\
\hline 16 & STUDY & 164 & SURE & 3 & TAPES \\
\hline $\begin{array}{r}8 \\
77\end{array}$ & $\begin{array}{l}\text { STUDY ING } \\
\text { STUFF }\end{array}$ & $\begin{array}{r}33 \\
3\end{array}$ & $\begin{array}{l}\text { SURELY } \\
\text { SUREACE }\end{array}$ & $\begin{array}{r}3 \\
10\end{array}$ & $\begin{array}{l}\text { TASK } \\
\text { TASTE }\end{array}$ \\
\hline 2 & STUMP & 10 & SURGEON & 3 & TASTED \\
\hline 20 & STUPID & 3 & SURGEON`S & 4 & TASTES \\
\hline 12 & STYLE & 18 & SURGERY & 2 & TATTY \\
\hline 2 & STYLUS & 2 & SURPRISE & 9 & TAUGHT \\
\hline 2 & SUBCOMMITTEE & 22 & SURPRISED & 23 & TAX \\
\hline 51 & SUBJECT & 2 & SURPRISES & 26 & TEA \\
\hline 10 & SUBJECTS & 3 & SURPRISING & 2 & TEABAGS \\
\hline 3 & SUBMIT & 2 & SURPRISINGLY & 35 & TEACH \\
\hline 5 & SUBMITTED & 3 & SURREPTITIOUS & 20 & TEACHER \\
\hline 3 & SUBSTANTIAL & 4 & SURREPTITIOUSLY & 17 & TEACHERS \\
\hline 2 & SUBSTITUTE & 4 & SURROUNDED & 2 & TEACHER $S$ \\
\hline 3 & SUCCEEDED & 18 & SURVEY & 6 & TEACHES \\
\hline 2 & SUCCESS & 7 & SUSPECT & 82 & TEACHING \\
\hline $\begin{array}{r}10 \\
2\end{array}$ & $\begin{array}{l}\text { SUCCESSFUL } \\
\text { SUCCINCT }\end{array}$ & $\begin{array}{l}3 \\
6\end{array}$ & $\begin{array}{l}\text { SUSPECTED } \\
\text { SUSPICIOUS }\end{array}$ & $\begin{array}{l}2 \\
3\end{array}$ & TEACH INGROOMS \\
\hline 72 & SUCH & 2 & SWAPPING & 3 & TEARS \\
\hline 3 & SUCK & 4 & SWEAT & 7 & TECHNICAL \\
\hline 28 & SUDDENLY & 7 & SWEET & 2 & TECHNIQUE \\
\hline 3 & SUFFER & 6 & SWITCH & 3 & TECHNIQUES \\
\hline 2 & SUFFERED & 30 & SYLLABUS & 2 & TECHNOLOGICAL \\
\hline 2 & SUFFERS & 4 & SYMBOL & 2 & TEDI OUS \\
\hline 3 & SUFFICIENT & 2 & SYMBOL ICAL & 2 & TEENAGE \\
\hline 3 & SUFF ICIENTLY & 2 & SYMBOLISM & 11 & TELEPHONE \\
\hline 6 & SUGAR & 3 & SYMPATHY & 19 & TELEVISION \\
\hline 9 & SUGGEST & 4 & SYMP HONY & 119 & TELL \\
\hline 8 & SUGGESTED & 30 & SYMPTOM & 21 & TELLING \\
\hline
\end{tabular}


Appendix A (Continued)

\begin{tabular}{|c|c|c|c|c|c|}
\hline 12 & TELLS & 4 & THERE`RE & 5 & TIGHT \\
\hline $\begin{array}{l}6 \\
3\end{array}$ & $\begin{array}{l}\text { TELLY } \\
\text { TEMP ERATURE }\end{array}$ & $\begin{array}{l}280 \\
268\end{array}$ & $\begin{array}{l}\text { THERE' } S \\
\text { THESE }\end{array}$ & $\begin{array}{r}5 \\
40\end{array}$ & $\begin{array}{l}\text { TILES } \\
\text { TILL }\end{array}$ \\
\hline 3 & TEMPORARILY & 17 & THESIS & 415 & TIME \\
\hline $\begin{array}{l}9 \\
2\end{array}$ & $\begin{array}{l}\text { TEMPORARY } \\
\text { TEMPTATION }\end{array}$ & $\begin{array}{r}1365 \\
49\end{array}$ & $\begin{array}{l}\text { THEY } \\
\text { THEY D }\end{array}$ & $\begin{array}{l}48 \\
14\end{array}$ & $\begin{array}{l}\text { TIMES } \\
\text { TIMETAB LE }\end{array}$ \\
\hline 71 & TEN & 19 & THEY LL & 8 & TIMETABLES \\
\hline $\begin{array}{r}26 \\
2\end{array}$ & $\begin{array}{l}\text { TEND } \\
\text { TENDED }\end{array}$ & $\begin{array}{l}266 \\
120\end{array}$ & $\begin{array}{l}\text { THEY RE } \\
\text { THEY VE }\end{array}$ & $\begin{array}{l}4 \\
2\end{array}$ & $\begin{array}{l}\text { TIMETAB L ING } \\
\text { TIMING }\end{array}$ \\
\hline $\begin{array}{l}2 \\
5 \\
5\end{array}$ & $\begin{array}{l}\text { TENDING } \\
\text { TENDS } \\
\text { T'ENNIS }\end{array}$ & $\begin{array}{r}2 \\
7 \\
470\end{array}$ & $\begin{array}{l}\text { THICK } \\
\text { THIN } \\
\text { THING }\end{array}$ & $\begin{array}{l}9 \\
3 \\
2\end{array}$ & $\begin{array}{l}\text { TIN } \\
\text { TINNED } \\
\text { TINS }\end{array}$ \\
\hline $\begin{array}{l}2 \\
3 \\
3\end{array}$ & $\begin{array}{l}\text { TENSE } \\
\text { TENSION } \\
\text { TENT }\end{array}$ & $\begin{array}{r}322 \\
2 \\
2\end{array}$ & $\begin{array}{l}\text { THINGS } \\
\text { THINGUMMY } \\
\text { THING'S }\end{array}$ & $\begin{array}{l}8 \\
2 \\
7\end{array}$ & $\begin{array}{l}\text { TINY } \\
\text { TIPTOE } \\
\text { TIRED }\end{array}$ \\
\hline $\begin{array}{r}2 \\
3 \\
39\end{array}$ & $\begin{array}{l}\text { TENTATIVELY } \\
\text { TENTH } \\
\text { TERM }\end{array}$ & $\begin{array}{r}1190 \\
64 \\
23\end{array}$ & $\begin{array}{l}\text { THINK } \\
\text { THINKING } \\
\text { THINKS }\end{array}$ & $\begin{array}{r}2 \\
6 \\
4434\end{array}$ & $\begin{array}{l}\text { TIRING } \\
\text { TITLE } \\
\text { TO }\end{array}$ \\
\hline 2 & TERMINAL & 27 & THIRD & 2 & TOAST \\
\hline $\begin{array}{r}2 \\
42\end{array}$ & $\begin{array}{l}\text { PERM INOLOGY } \\
\text { TERMS }\end{array}$ & $\begin{array}{r}2 \\
10\end{array}$ & $\begin{array}{l}\text { THIRDLY } \\
\text { THIRTEEN }\end{array}$ & $3 \frac{1}{2}$ & $\begin{array}{l}\text { TODAY } \\
\text { TODDLED }\end{array}$ \\
\hline $\begin{array}{r}2 \\
34 \\
66\end{array}$ & $\begin{array}{l}\text { TERMT IME } \\
\text { TERRIBLE } \\
\text { TERRIBLY }\end{array}$ & $\begin{array}{r}3 \\
4 \\
32\end{array}$ & $\begin{array}{l}\text { THIRTEENTH } \\
\text { THIRTIES } \\
\text { THIRTY }\end{array}$ & $\begin{array}{r}30 \\
10 \frac{1}{2}\end{array}$ & $\begin{array}{l}\text { TOGETHER } \\
\text { TOLD } \\
\text { TOLLS }\end{array}$ \\
\hline $\begin{array}{l}3 \\
7 \\
6\end{array}$ & $\begin{array}{l}\text { TERRIFIC } \\
\text { TERRIFYING } \\
\text { TEST }\end{array}$ & $\begin{array}{l}5 \\
4 \\
5\end{array}$ & $\begin{array}{l}\text { THIRTYFIVE } \\
\text { THIRTYFOUR } \\
\text { THIRTYNINE }\end{array}$ & $\begin{array}{r}25 \\
3 \\
6\end{array}$ & $\begin{array}{l}\text { TOMORROW } \\
\text { TON } \\
\text { TONE }\end{array}$ \\
\hline 2 & TESTING & 2 & THIRTYONE & 4 & TONGUE \\
\hline 3 & TESTS & 2 & THIRTYSIX & 9 & TONIGHT \\
\hline 9 & TEXT & 8 & THIRTYTHREE & 197 & TOO \\
\hline 4 & TEXTBOOK & 5 & THIRTYTWO & 49 & TOOK \\
\hline $\begin{array}{r}2 \\
24\end{array}$ & $\begin{array}{l}\text { TEXTBOOKS } \\
\text { TEXTS }\end{array}$ & $\begin{array}{r}1582 \\
2\end{array}$ & $\begin{array}{l}\text { THIS } \\
\text { THIS`LL }\end{array}$ & $\begin{array}{l}2 \\
2\end{array}$ & $\begin{array}{l}\text { TOOL } \\
\text { TOOTH }\end{array}$ \\
\hline 233 & THAN & 3 & THOROUGHLY & 27 & TOP \\
\hline 76 & THANK & 153 & THOSE & 3 & TOPIC \\
\hline 2 & THANKED & 110 & THOUGH & 2 & TOPICS \\
\hline 23 & THANKS & 320 & THOUGHT & 3 & TORN \\
\hline $\begin{array}{r}3169 \\
7\end{array}$ & $\begin{array}{l}\text { THAT } \\
\text { THAT D }\end{array}$ & $\begin{array}{r}58 \\
2\end{array}$ & $\begin{array}{l}\text { THOU SAND } \\
\text { THOU SANDS }\end{array}$ & $\begin{array}{l}8 \\
2\end{array}$ & $\begin{array}{l}\text { TOTAL } \\
\text { TOTALITARIAN }\end{array}$ \\
\hline 10 & THAT' LL & 2 & THRASHED & 20 & TOTALLY \\
\hline 757 & THAT $\mathrm{S}$ & 252 & THREE & 17 & TOUCH \\
\hline 6833 & THE & 4 & THRESHOLD & 2 & TOUCHED \\
\hline 17 & THEATRE & 4 & THREW & 2 & TOUCHING \\
\hline 3 & THEATRICAL & 2 & THROAT & 8 & TOUGH \\
\hline 203 & THEIR & 136 & THROUGH & 5 & TOUGHER \\
\hline 4 & THEIRS & 6 & THROUGHOUT & 5 & TOUR \\
\hline $\begin{array}{r}493 \\
11\end{array}$ & $\begin{array}{l}\text { THEM } \\
\text { THEME }\end{array}$ & $\begin{array}{l}5 \\
2\end{array}$ & $\begin{array}{l}\text { THROW } \\
\text { THROWAWAY }\end{array}$ & $\begin{array}{l}18 \\
14\end{array}$ & $\begin{array}{l}\text { TOWARDS } \\
\text { TOWN }\end{array}$ \\
\hline 2 & THEMES & 10 & THROWN & 3 & TOWNS \\
\hline $\begin{array}{r}34 \\
594\end{array}$ & $\begin{array}{l}\text { THEMSELVES } \\
\text { THEN }\end{array}$ & $\begin{array}{l}3 \\
6\end{array}$ & $\begin{array}{l}\text { THROWS } \\
\text { THUMB }\end{array}$ & $\begin{array}{l}5 \\
3\end{array}$ & $\begin{array}{l}\text { TRACK } \\
\text { TRACKS }\end{array}$ \\
\hline $\begin{array}{r}5 \\
11 \\
3\end{array}$ & $\begin{array}{l}\text { THEORIES } \\
\text { THEORY } \\
\text { THERAPEUTICS }\end{array}$ & $\begin{array}{r}2 \\
11 \\
6\end{array}$ & $\begin{array}{l}\text { THUMBNAII } \\
\text { THURSDAY } \\
\text { TICK }\end{array}$ & $\begin{array}{l}6 \\
7 \\
2\end{array}$ & $\begin{array}{l}\text { TRADE } \\
\text { TRADITION } \\
\text { TRADITIONS }\end{array}$ \\
\hline 875 & THERE & 6 & TICKET & 10 & TRAGEDIES \\
\hline 2 & THEREAFTER & 6 & TICKETS & 27 & TRAGEDY \\
\hline 24 & THEREFORE & 2 & TICKS & 3 & TRAG IC \\
\hline 3 & THEREOF & 7 & TIDY & 15 & TRAIN \\
\hline $\begin{array}{l}9 \\
5\end{array}$ & $\begin{array}{l}\text { THERE D } \\
\text { THERE LL }\end{array}$ & $\begin{array}{l}4 \\
6\end{array}$ & $\begin{array}{l}\text { TIE } \\
\text { TIED }\end{array}$ & $\begin{array}{r}4 \\
12\end{array}$ & $\begin{array}{l}\text { TRAINED } \\
\text { TRAINING }\end{array}$ \\
\hline
\end{tabular}


Appendix A (Continued)

\begin{tabular}{|c|c|c|c|c|c|}
\hline 7 & TRAINS & 43 & TWENTY & 108 & UNIVERSITY \\
\hline 3 & TRANSCRIPTION & 9 & TWENTYEIGH'T & 2 & UNJUST \\
\hline 2 & TRANSCRIPTIONS & 2 & TWENTYEIGHTH & 3 & UNKNOWN \\
\hline $\begin{array}{l}3 \\
2\end{array}$ & $\begin{array}{l}\text { TRANSCRIPTS } \\
\text { TRANSFORMATION }\end{array}$ & $\begin{array}{r}3 \\
17\end{array}$ & $\begin{array}{l}\text { TWENTYF IFTH } \\
\text { TWENTYF IVE }\end{array}$ & $\begin{array}{r}27 \\
2\end{array}$ & $\begin{array}{l}\text { UNLESS } \\
\text { UNLIKE }\end{array}$ \\
\hline 3 & TRANSLATE & 4 & TWENTYFOUR & 6 & UNLIKELY \\
\hline 3 & TRANSLATION & 4 & TWENTYFOURTH & 3 & UNNERVING \\
\hline 3 & TRANSLATIONS & 7 & TWENTYNINE & 5 & UNPLEASANT \\
\hline 4 & TRANSPIRED & 6 & TWENTYNINTH & 2 & UNPREDICTABLE \\
\hline $\begin{array}{l}2 \\
4\end{array}$ & $\begin{array}{l}\text { TRANSPORT } \\
\text { TRASH }\end{array}$ & $\begin{array}{l}5 \\
6\end{array}$ & $\begin{array}{l}\text { TWENTYONE } \\
\text { TWENTYSEVEN }\end{array}$ & $\begin{array}{l}2 \\
3\end{array}$ & $\begin{array}{l}\text { UNREFLECTIVE } \\
\text { UNRELIABIE }\end{array}$ \\
\hline 2 & TRAUMAS & 7 & TWENTYSIX & 3 & UNSAT I SFACTORY \\
\hline 10 & TRAVEL & 6 & TWENTYTHREE & 2 & UNSHAKEABLE \\
\hline 2 & TRAVELLED & 8 & TWENTYTWO & 2 & UNTIED \\
\hline 5 & TRAVELLING & 15 & TWICE & $6 !$ & UNTIL \\
\hline $\begin{array}{l}8 \\
3\end{array}$ & $\begin{array}{l}\text { TREAT } \\
\text { TREATED }\end{array}$ & $\begin{array}{l}2 \\
3\end{array}$ & $\begin{array}{l}\text { TWIDDLE } \\
\text { TWIST }\end{array}$ & $\begin{array}{l}2 \\
2\end{array}$ & $\begin{array}{l}\text { UNTOUCHED } \\
\text { UNUSED }\end{array}$ \\
\hline 3 & TREATING & 2 & TWITTERED & 7 & UNUSUAL \\
\hline $\begin{array}{l}3 \\
5\end{array}$ & $\begin{array}{l}\text { TREATMENT } \\
\text { TREE }\end{array}$ & $\begin{array}{r}36 \overline{3} \\
34\end{array}$ & $\begin{array}{l}\text { TWO } \\
\text { TYPE }\end{array}$ & $\begin{array}{r}592 \\
2\end{array}$ & $\begin{array}{l}\text { UP } \\
\text { UPHELD }\end{array}$ \\
\hline 5 & TREES & 2 & TYPECAST & 15 & UPON \\
\hline $\begin{array}{l}6 \\
4\end{array}$ & $\begin{array}{l}\text { TREMENDOUS } \\
\text { TRENCHES }\end{array}$ & $\begin{array}{l}3 \\
2\end{array}$ & $\begin{array}{l}\text { TYPES } \\
\text { TYPESCRIPT }\end{array}$ & $\begin{array}{l}3 \\
4\end{array}$ & $\begin{array}{l}\text { UPPER } \\
\text { UPSET }\end{array}$ \\
\hline 3 & TREND & 3 & TYPICAL & 4 & UPSIDE \\
\hline 4 & IRIAL & 6 & TYPING & 15 & UPSTAIRS \\
\hline 3 & TRIBES & 5 & $\mathrm{U}$ & 151 & US \\
\hline 2 & TRIBUTE & 4 & UGH & 70 & USE \\
\hline 2 & TRICKY & 2 & UGLY & 123 & USED \\
\hline 35 & PRIED & 2 & $\mathrm{UH}$ & 12 & USEFUL \\
\hline $\begin{array}{l}3 \\
5\end{array}$ & $\begin{array}{l}\text { TRINITY } \\
\text { TRIP }\end{array}$ & $\begin{array}{l}7 \\
2\end{array}$ & $\begin{array}{l}\text { UHUH } \\
\text { ULT IMATELY }\end{array}$ & $\begin{array}{l}3 \\
9\end{array}$ & $\begin{array}{l}\text { USELESS } \\
\text { USES }\end{array}$ \\
\hline 8 & TRIVIAL & 2 & UN & 21 & USING \\
\hline 4 & TROOPS & 2 & UNBEARAB LE & 15 & USUAL \\
\hline 2 & TROT & 6 & UNBELIEVABLE & 18 & USUALLY \\
\hline 55 & TROUBLE & 3 & UNCERTAIN & 6 & UTTERLY \\
\hline 2 & TROUBLED & 3 & UNCLE & 2 & $\mathrm{~V}$ \\
\hline 7 & TROUBLES & 2 & UNCOMFORTABLE & 4 & VAC \\
\hline 2 & TROU SERS & 43 & UNDER & 2 & VACATION \\
\hline 83 & TRUE & 16 & UNDERGRADUATE & 11 & VAGUE \\
\hline $\begin{array}{l}4 \\
3\end{array}$ & $\begin{array}{l}\text { TRUST } \\
\text { TRUSTWORTHY }\end{array}$ & $\begin{array}{l}15 \\
44\end{array}$ & $\begin{array}{l}\text { UNDERGRADUATES } \\
\text { UNDERSTAND }\end{array}$ & $\begin{array}{l}7 \\
3\end{array}$ & $\begin{array}{l}\text { VAGUELY } \\
\text { VALLEY }\end{array}$ \\
\hline 7 & TRUTH & 3 & UNDERSTANDING & 3 & VALUABLE \\
\hline 64 & TRY & 3 & UNDERSTANDS & 3 & VALUATION \\
\hline 83 & TRYING & 5 & UNDERSTOOD & 13 & VALUE \\
\hline 2 & TS & 3 & UNEMP LOYED & 3 & VALUED \\
\hline 3 & TUBE & 2 & UNEMP LOYMENT & 2 & $\begin{array}{l}\text { VANISH } \\
\text { VARIATIONS }\end{array}$ \\
\hline 11 & TUESDAY & 2 & UNEXPECTED & 2 & VARIATIONS \\
\hline $\begin{array}{r}2 \\
26\end{array}$ & $\begin{array}{l}\text { TUNE } \\
\text { TURN }\end{array}$ & 5 & UNFAIR & 3 & VARIED \\
\hline 28 & $\begin{array}{l}\text { TURN } \\
\text { TURNED }\end{array}$ & $\begin{array}{r}8 \\
10\end{array}$ & $\begin{array}{l}\text { UNFORTUNATE } \\
\text { UNFORTUNATELY }\end{array}$ & $\begin{array}{l}2 \\
5\end{array}$ & $\begin{array}{l}\text { VARIES } \\
\text { VARIETY }\end{array}$ \\
\hline 7 & TURNING & 2 & UNHEALTHY & 30 & VARIOUS \\
\hline 4 & TURNS & 7 & UNIFORM & 6 & VARY \\
\hline 5 & TUSK & 2 & UNIFORMS & 8 & VAST \\
\hline 19 & TUTOR & 5 & UNION & 6 & VERB \\
\hline 4 & TUTORIALS & 2 & UNIQUE & 5 & VERBAL \\
\hline 2 & TUTORS & 2 & UNISEX & 6 & VERBS \\
\hline 2 & TWELFTH & 18 & UNIT & 3 & VERSA \\
\hline 29 & TWELVE & 13 & UNITED & 3 & VERSE \\
\hline 5 & TWENTIES & 2 & UNIVERSAL & 6 & VERSION \\
\hline 5 & TWENT IETH & 13 & UNIVERSITIES & 2 & VERSIONS \\
\hline
\end{tabular}


Appendix A (Continued)

\begin{tabular}{|c|c|c|c|c|c|}
\hline 982 & VERY & 2 & WARMTH & 3 & WHEREABOUTS \\
\hline $\begin{array}{l}3 \\
2 \\
3\end{array}$ & $\begin{array}{l}\text { VICE } \\
\text { VICECHANCELLOR } \\
\text { VICEPRESBYTERS }\end{array}$ & $\begin{array}{l}2 \\
4 \\
7\end{array}$ & $\begin{array}{l}\text { WARN } \\
\text { WARNING } \\
\text { WARS }\end{array}$ & $\begin{array}{r}21 \\
4 \\
11\end{array}$ & $\begin{array}{l}\text { WHEREAS } \\
\text { WHEREBY } \\
\text { WHEREVER }\end{array}$ \\
\hline 3 & VICEROY & 4 & WART IME & 8 & WHERE'S \\
\hline $\begin{array}{l}2 \\
3\end{array}$ & $\begin{array}{l}\text { VICTIMS } \\
\text { VICTORIA }\end{array}$ & $\begin{array}{r}2079 \\
6\end{array}$ & $\begin{array}{l}\text { WAS } \\
\text { WASH }\end{array}$ & $\begin{array}{l}120 \\
636\end{array}$ & $\begin{array}{l}\text { WHETHER } \\
\text { WHICH }\end{array}$ \\
\hline 3 & VIDEO & 2 & WASHES & 43 & WHILE \\
\hline 43 & VIEW & 4 & WASHING & 6 & WHIPPED \\
\hline 3 & VIEWPOINT & 6 & WASHINGUP & 2 & WHISKED \\
\hline $\begin{array}{r}10 \\
2\end{array}$ & $\begin{array}{l}\text { VIEWS } \\
\text { VIKING }\end{array}$ & $\begin{array}{r}142 \\
13\end{array}$ & $\begin{array}{l}\text { WASN'T } \\
\text { WASTE }\end{array}$ & $\begin{array}{l}4 \\
2\end{array}$ & $\begin{array}{l}\text { WHISKY } \\
\text { WHISTIE }\end{array}$ \\
\hline 7 & VILLAGE & $\begin{array}{r}13 \\
6\end{array}$ & $\begin{array}{l}\text { WASTE } \\
\text { WASTED }\end{array}$ & $\begin{array}{l}2 \\
19\end{array}$ & $\begin{array}{l}\text { WHISTLE } \\
\text { WHITE }\end{array}$ \\
\hline 2 & VINTAGE & 4 & WAST ING & 2 & WHITSUN \\
\hline 8 & VIRTUALLY & 7 & WATCH & 2 & WHIZZING \\
\hline 2 & VISA & 9 & WATCHING & 395 & WHO \\
\hline 3 & VISAVIS & 24 & WATER & 2 & WHOA \\
\hline 3 & VISION & 2 & WAVE & 8 & WHOEVER \\
\hline 10 & VISI'T & 282 & WAY & 136 & WHOLE \\
\hline 4 & VISITED & 25 & WAYS & 18 & WHOM \\
\hline 3 & VISITOR & 1060 & WE & 4 & WHORE \\
\hline 2 & VISITORS & 2 & WEAK & 17 & WHOSE \\
\hline 3 & VISITS & 3 & WEAKEST & 10 & WHO`D \\
\hline 7 & VISUAL & 4 & WEALTHY & 2 & WHO`LL \\
\hline 16 & VITAMIN & 11 & WEAR & 56 & WHO'S \\
\hline 7 & VIVA & 8 & WEAR ING & 7 & WHO $\mathrm{VE}$ \\
\hline 4 & VIVAS & 2 & WEARY & 201 & WHY \\
\hline 2 & VIVID & 6 & WEATHER & 2 & WICKED \\
\hline 3 & VOCABULARY & 8 & WEDDING & 7 & WIDE \\
\hline 10 & VOICE & 2 & WEDLOCK & 6 & WIDELY \\
\hline 4 & VOICES & 18 & WEDNESDAY & 3 & WIDER \\
\hline 7 & VOLUME & 2 & WEDNESDAYS & 3 & WIDOW \\
\hline 3 & VOLUNTARY & 82 & WEEK & 2 & WIDOWER \\
\hline 2 & VON & 13 & WEEKEND & 22 & WIFE \\
\hline 9 & VOTE & 3 & WEEKENDS & 2 & WIGGLE \\
\hline 22 & $w$ & 63 & WEEKS & 10 & WILD \\
\hline 17 & WAIT & 2 & WEEK $S$ & 2 & WILDLY \\
\hline 5 & WAITED & 2 & WEIGHTING & 197 & WILL \\
\hline 16 & WAITING & 5 & WEIRD & 11 & WILLING \\
\hline 2 & WAKE & 5 & WELCOME & 3 & WIN \\
\hline 19 & WALK & 1753 & WELL & 9 & WIND \\
\hline 26 & WALKED & 4 & WELLK NOWN & 2 & WINDAN \\
\hline 13 & WALK ING & 218 & WENT & 6 & WINDING \\
\hline 4 & WALKS & 539 & WERE & 4 & WINDOW \\
\hline 13 & WALL & 36 & WEREN`T & 2 & WINDOWS \\
\hline 2 & WALLAHS & 12 & WEST & 15 & WINE \\
\hline 9 & WALLS & 3 & WET & 4 & WINES \\
\hline 2 & WANDER & 2 & WETTEX & 2 & WINGS \\
\hline 4 & WANDERED & 28 & $W E^{`} D$ & 11 & WINTER \\
\hline 6 & WANDERING & 44 & WE`LL & 4 & WIRE \\
\hline 2 & WANGLE & 116 & WE'RE & 4 & $\begin{array}{l}\text { WIRELESS } \\
\text { WIRING }\end{array}$ \\
\hline 251 & WANT & 112 & $W E^{\sim} \mathrm{VE}$ & 2 & WIRING \\
\hline 81 & WANTED & 1119 & WHAT & 6 & WISE \\
\hline 10 & WANT ING & 53 & WHATEVER & 19 & WISH \\
\hline 34 & WANTS & 116 & WHAT'S & 845 & WITH \\
\hline 49 & WAR & 2 & WHEELCHAIRS & 2 & WITHDRAW \\
\hline 3 & WARD & 2 & WHEELS & 2 & WITHORAWN \\
\hline 2 & WARDROBE & 557 & WHEN & 39 & WITHIN \\
\hline $\begin{array}{r}3 \\
4\end{array}$ & $\begin{array}{l}\text { WAREHOUSE } \\
\text { WARM }\end{array}$ & $\begin{array}{r}12 \\
251\end{array}$ & $\begin{array}{l}\text { WHENEVER } \\
\text { WHERE }\end{array}$ & 51 & $\begin{array}{l}\text { WITHOUT } \\
\text { WITS }\end{array}$ \\
\hline
\end{tabular}


Appendix A (Continued)

\begin{tabular}{|c|c|c|c|c|c|}
\hline 2 & WITTY & 15 & WORRY & 199 & YEARS \\
\hline 2 & WIVES & 4 & WORRYING & 4 & YEARS \\
\hline 6 & WOK & 16 & WORSE & 3 & YEAR'S \\
\hline 2 & WOLF & 4 & WORST & 2 & YEAST \\
\hline 33 & WOMAN & 65 & WORTH & 2 & YELLOW \\
\hline 2 & WOMAN`S & 5 & WORTHWH ILE & 6 & YEP \\
\hline 26 & WOMEN & 4 & WORTHY & 2675 & YES \\
\hline 3 & WOMEN'S & 697 & WOULD & 12 & YEST ERDAY \\
\hline 20 & WONDER & 142 & WOULDN'T & 69 & YET \\
\hline 10 & WONDERED & 3 & WOW & 9 & YIDDISH \\
\hline 14 & WONDERFUL & 5 & WRETCHED & 4817 & YOU \\
\hline 7 & WONDERING & 53 & WRITE & 32 & YOUNG \\
\hline 51 & WON'T & 5 & WRITER & 14 & YOUNGER \\
\hline 5 & WOOD & 2 & WRITERS & 2 & YOUNGEST \\
\hline 2 & WOODEN & 5 & WRITES & 424 & YOUR \\
\hline 6 & WOOLLY & 56 & WRIT ING & 13 & YOURS \\
\hline 52 & WORD & 44 & WRITTEN & 36 & YOURSELF \\
\hline 55 & WORDS & 51 & WRONG & 2 & YOUTH \\
\hline 3 & WORE & 32 & WROTE & 74 & YOU D \\
\hline 192 & WORK & 5 & $x$ & 48 & YOU 'LL \\
\hline 28 & WORKED & 14 & XEROX & 271 & YOU 'RE \\
\hline 4 & WORKER & 6 & XRAY & 218 & $\mathrm{YOU}^{\prime} \mathrm{VE}$ \\
\hline 3 & WORKERS & 2 & XRAYS & 6 & YUP \\
\hline 64 & WORKING & 6 & $Y$ & 2 & $\mathrm{z}$ \\
\hline 30 & WORKS & 2 & YACHT ING & 5 & ZERO \\
\hline 53 & WORLD & 3 & YARDS & 8 & ZOOLOGY \\
\hline 2 & WORLD`S & 22 & YEA & 6 & EM \\
\hline 9 & WORRIED & 854 & YEAH & & \\
\hline 6 & WORRIES & 203 & YEAR & & \\
\hline
\end{tabular}

Appendix B

Rank Ordering of Words With Frequencies Greater Than 150 in Svartvik and Quirk (1980)

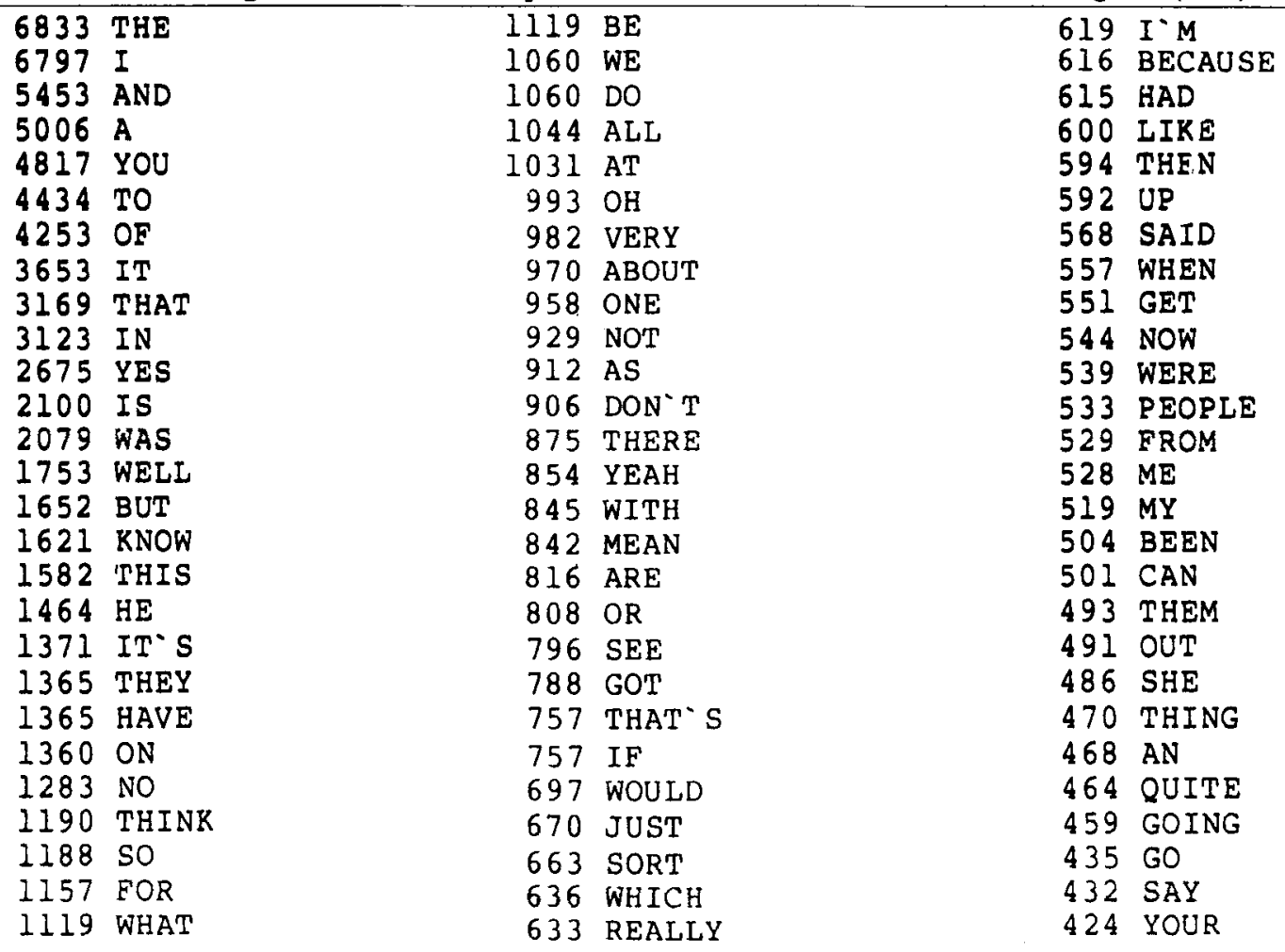


Appendix B (Continued)

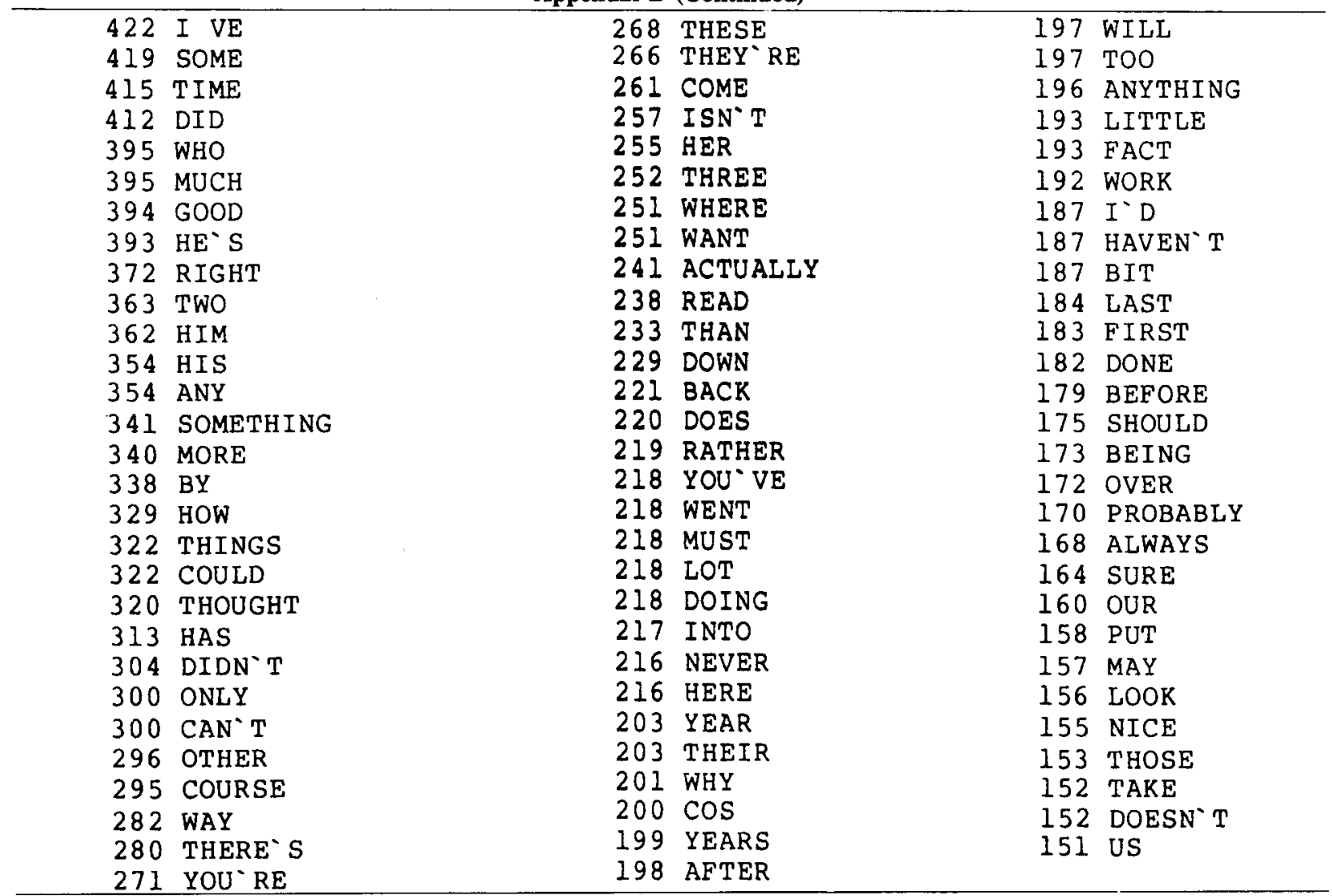

(Manuscript received June 8, 1984;

revision accepted for publication December 10, 1984.) 